\title{
General Access to Concave-Substituted cis- Dioxabicyclo[3.3.0]octanones: Enantioselective Total Syntheses of Macfarlandin C and Dendrillolide A
}

Tyler K. Allred, André P. Dieskau, Peng Zhao, Gregory L. Lackner, and Larry E. Overman* Department of Chemistry, University of California, Irvine, California 92697-2025

\section{Supporting Information - Table of Contents}

Summary of Unsuccessful Hydrogenation Conditions for the Conversion of 17 to 19/21 or 18

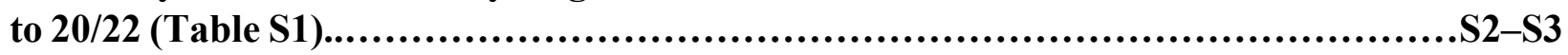

Summary of Unsuccessful HAT Hydrogenation Conditions for the Conversion of 18 to 20/22

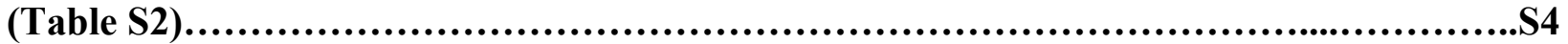

Comparison of ${ }^{1} \mathrm{H}$ and ${ }^{13} \mathrm{C}$ NMR of Natural and Synthetic Macfarlandin C (Table S3) .55

Comparison of ${ }^{13} \mathrm{C}$ NMR of Natural and Synthetic Dendrillolide A (Table S4). S7-S10

X-Ray Structure of Mukaiyama-Hydration Product 32.

References.....................................................................................S9

Spectral Data...............................................................................S10-S99 
Table S1: Summary of Unsuccessful Hydrogenation Conditions for the Conversion of 17 to 19/21 or 18 to $20 / 22$

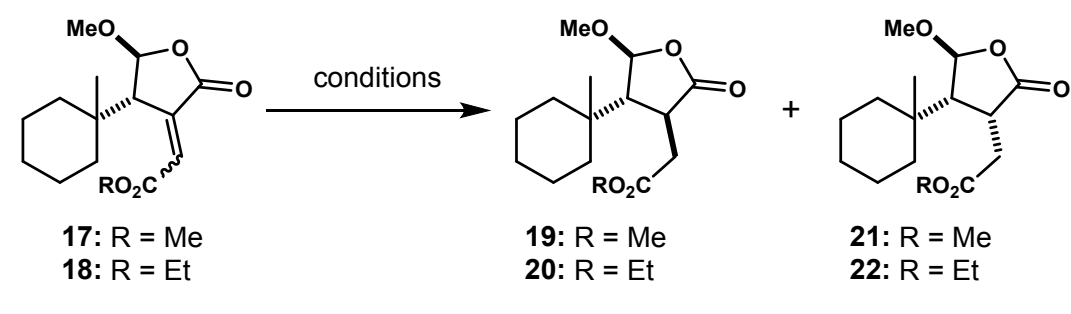

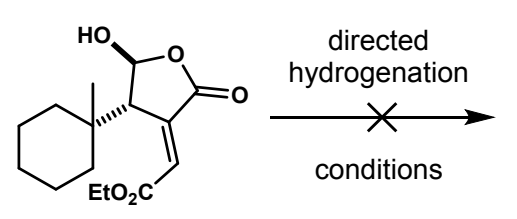

23

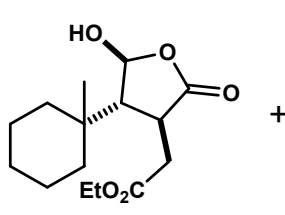

24

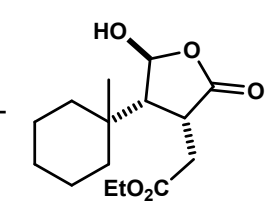

25

\begin{tabular}{|c|c|c|c|}
\hline Entry & SM & Conditions & 3,4-trans:3,4-cis ${ }^{a}$ \\
\hline 1 & 17 & cat. $\mathrm{Pd} / \mathrm{C}, \mathrm{H}_{2}(1 \mathrm{~atm}), \mathrm{MeOH}, \mathrm{rt}$ & $7: 1$ \\
\hline 2 & 18 & cat. $\mathrm{PtO}_{2}, \mathrm{H}_{2}(1 \mathrm{~atm}), \mathrm{EtOAc}, \mathrm{rt}$ & $1.5: 1$ \\
\hline 3 & 17 & cat. $\mathrm{Rh} / \mathrm{Al}_{2} \mathrm{O}_{3}, \mathrm{H}_{2}(15 \mathrm{~atm}), \mathrm{EtOAc}, \mathrm{rt}$ & 3:1 \\
\hline 4 & 17 & cat. $\mathrm{Rh}\left(\mathrm{PPh}_{3}\right)_{3} \mathrm{Cl}, \mathrm{H}_{2}(7 \mathrm{~atm}) \mathrm{C}_{6} \mathrm{H}_{6}, \mathrm{rt}$ & $-b$ \\
\hline 5 & 17 & cat. $\left[\operatorname{Ir}(\mathrm{COD})(\mathrm{pyr}) \mathrm{PCy}_{3}\right]\left(\mathrm{PF}_{6}\right), \mathrm{H}_{2}(85 \mathrm{~atm}), \mathrm{CH}_{2} \mathrm{Cl}_{2}, \mathrm{rt}$ & $-b$ \\
\hline 6 & 17 & $\mathrm{~N}_{2} \mathrm{H}_{4}, \mathrm{PhI}(\mathrm{OAc})_{2}, \mathrm{H}_{2} \mathrm{O}, \mathrm{CH}_{2} \mathrm{Cl}_{2}, \mathrm{rt}$ & $-\mathrm{b}$ \\
\hline 7 & 18 & $\mathrm{TsNHNH}_{2}, \mathrm{THF}, \mathrm{NaOAc}, \mathrm{H}_{2} \mathrm{O}$ & $1.2: 1$ \\
\hline 8 & 17 & IPr-CuCl, $\mathrm{NaO} t-\mathrm{Bu}, \mathrm{PMHS}, \mathrm{PhMe}, \mathrm{rt}$ & 1:0 \\
\hline 9 & 17 & Red-Al, CuI, 2-butanol, THF/PhMe, -78 to $-20{ }^{\circ} \mathrm{C}$ & 1.0:1.3 \\
\hline 10 & 17 & $\mathrm{NaBH}_{4}, \mathrm{NiCl}_{2}, \mathrm{MeOH} 0{ }^{\circ} \mathrm{C}$ & 2:1 \\
\hline 11 & 17 & L-selectride, THF, $-78^{\circ} \mathrm{C}$ & $1: 0$ \\
\hline 12 & 18 & L-selectride, THF, $-78^{\circ} \mathrm{C}$, then $\mathrm{H}_{2} \mathrm{O}$ & 10:1 \\
\hline 13 & 18 & L-selectride, THF, $-78^{\circ} \mathrm{C}$, then BHT & 1:0 \\
\hline 14 & 18 & L-selectride, THF, $-78^{\circ} \mathrm{C}$, then $\mathrm{N}-i \operatorname{Pr}-(-)$-ephedrine & 1:0 \\
\hline 15 & 18 & L-selectride, THF, $-78^{\circ} \mathrm{C}$, then $\mathrm{N}-i \operatorname{Pr}-(+)$-ephedrine & 1:0 \\
\hline
\end{tabular}


$1723 \quad \mathrm{Rh}(\mathrm{OAc})_{2},(\mathrm{~S})-\mathrm{BINAP}, \mathrm{CH}_{2} \mathrm{Cl}_{2}, \mathrm{H}_{2}(110 \mathrm{~atm}) \quad$-b

$1823 \quad\left[\operatorname{Ir}(\operatorname{cod}) p y\left(\mathrm{PCy}_{3}\right)\right] \mathrm{PF}_{6}, \mathrm{~B}(\mathrm{O} i \mathrm{Pr})_{3}, \mathrm{CH}_{2} \mathrm{Cl}_{2}, \mathrm{H}_{2}(110$ atm)

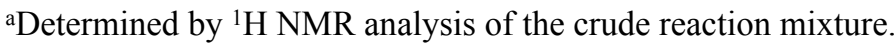

bNo conversion was observed.

${ }^{\mathrm{c}}$ Conversion to an intractable mixture.

IPr-CuCl = N,N-bis(2,6-diisopropylphenyl)imidazol-2-ylidene copper(I) chloride. RedAl = sodium bis-(2methoxyethoxy)aluminum hydride. L-Selectride $=$ lithium tri-sec-butylborohydride. PMHS = polymethylhydrosiloxane. BHT = 2,6-di-tert-butyl-4-methylphenol. 
Table S2: Summary of Unsuccessful HAT Hydrogenation Conditions for the Conversion of $\mathbf{1 8}$ to 20/22

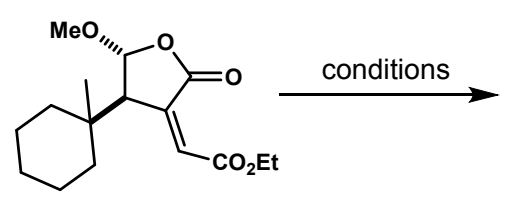

$(Z)-18$

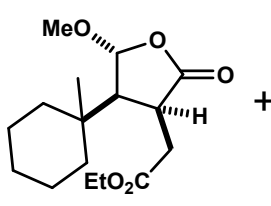

20

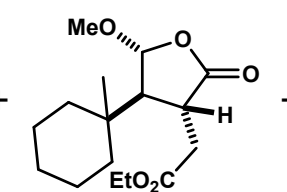

22

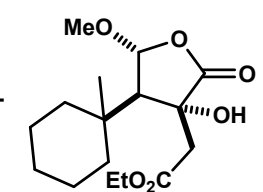

32

\section{Conditions $^{1-5}$}

Yielda,b

$d r(20: 22)^{c}$

$\mathrm{CoCl}_{2} \cdot 6 \mathrm{H}_{2} \mathrm{O}(1 \mathrm{eq}), \mathrm{NaBH}_{4}(2 \mathrm{eq}), \mathrm{EtOH}(0.1 \mathrm{M}), 0{ }^{\circ} \mathrm{C}, 3 \mathrm{~h}$

$27 \%(0 \%)$

$1: 8$

$\mathrm{Co}(\mathrm{acac})_{2}(1 \mathrm{eq}), t$ - $\mathrm{BuOOH}(3 \mathrm{eq}), \mathrm{Et}_{3} \mathrm{SiH}(10 \mathrm{eq}), 1,4-$ cyclohexadiene (10 eq), $i-\operatorname{PrOH}(0.1 \mathrm{M}), \mathrm{rt}, 3 \mathrm{~h}$

$10 \%(8 \%)$

$\mathrm{Co}(\mathrm{dpm})_{2}(10 \mathrm{~mol} \%), \mathrm{PhSiH}_{3}$ (3 eq), $t-\mathrm{BuOOH}$ (3 eq), $\mathrm{PhCF}_{3}(0.1 \mathrm{M}), \mathrm{rt}, 18 \mathrm{~h}$

$0 \%(50 \%)$

$80 \%(15 \%)$

$1: 7$

$\mathrm{Mn}(\mathrm{dpm})_{3}(3 \mathrm{~mol} \%), \mathrm{PhSiH}_{3}(1.5 \mathrm{eq}), \mathrm{CH}_{2} \mathrm{Cl}_{2} / i-\mathrm{PrOH}(4: 1,0.1 \mathrm{M})$ $\mathrm{rt}, 18 \mathrm{~h}$

$40 \%(12 \%)$

$\mathrm{Mn}(\mathrm{dpm})_{3}(10 \mathrm{~mol} \%), \mathrm{PhSiH}_{3}$ (3 eq), $t$ - $\mathrm{BuOOH}(3 \mathrm{eq})$, $i$-PrOH $(0.1 \mathrm{M}), \mathrm{rt}, 18 \mathrm{~h}$

$24 \%(8 \%)$

$\mathrm{Mn}(\mathrm{dpm})_{3}$ (3 mol\%), $\mathrm{Ph}\left(\mathrm{O} i\right.$-Pr)SiH${ }_{2}(3 \mathrm{eq}), t-\mathrm{BuOOH}$ (3 eq), hexanes $(0.1 \mathrm{M}), \mathrm{rt}, 18 \mathrm{~h}$

${ }^{a}$ Determined by ${ }^{1} \mathrm{H}$ NMR integration relative to an internal standard (1,2-dibromo-4,5methylenedioxybenzene).

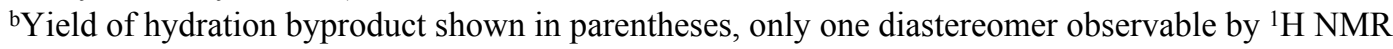

'Diastereomeric ratio determined by comparing integrations of acetal proton 
Table S3: Comparison of ${ }^{1} \mathrm{H}$ and ${ }^{13} \mathrm{C}$ NMR of Natural and Synthetic Macfarlandin C.

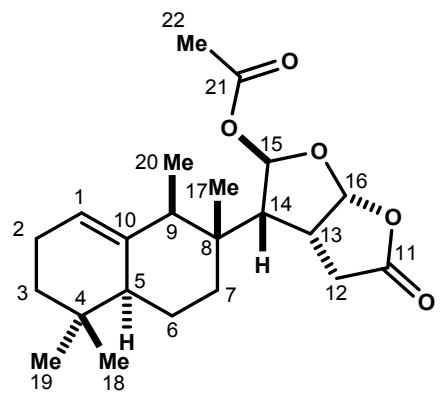

macfarlandin C

${ }^{1} \mathrm{H}(600 \mathrm{MHz})$ and ${ }^{13} \mathrm{C}(150 \mathrm{MHz})^{6,8}$

Solvent: $\mathbf{C D C l}_{3}$

\begin{tabular}{|c|c|c|c|c|}
\hline Atom & ${ }^{1}$ H Shift Exp. & ${ }^{1} \mathrm{H}$ Shift Lit. & $\begin{array}{c}{ }^{13} \text { C Shift } \\
\text { Exp. }\end{array}$ & $\begin{array}{c}{ }^{13} \text { C Shift } \\
\text { Lit. }\end{array}$ \\
\hline 1 & $5.31(\mathrm{brt}, 4.0 \mathrm{~Hz})$ & $5.32(\mathrm{br} \mathrm{t}, 4.0 \mathrm{~Hz})$ & 118.4 & 118.3 \\
\hline 2 & $2.05-1.98(\mathrm{~m})$ & $2.05(\mathrm{~m})$ & 23.0 & 23.0 \\
\hline $3 \mathrm{a}$ & $1.36-1.31(\mathrm{~m}, 1 \mathrm{H})$ & $1.36(\mathrm{ddd}, 13,7.5,6.9 \mathrm{~Hz})$ & 32.6 & 32.6 \\
\hline $3 \mathrm{~b}$ & $1.15(\mathrm{dt}, 4.8,12.8 \mathrm{~Hz})$ & $1.16(\mathrm{dt}, 4.6,13.0 \mathrm{~Hz})$ & 32.6 & 32.6 \\
\hline 4 & NA & NA & 31.2 & 31.2 \\
\hline 5 & $1.43-1.37(\mathrm{~m})$ & Not Reported & 49.0 & 49.0 \\
\hline $6 a$ & $1.76-1.69(\mathrm{~m})$ & Not Reported & 25.2 & 25.2 \\
\hline $6 \mathrm{~b}$ & $1.25-1.19(\mathrm{~m})$ & Not Reported & 25.2 & 25.2 \\
\hline 7 & $1.66-1.57(\mathrm{~m}, 2 \mathrm{H})$ & Not Reported & 35.5 & 35.4 \\
\hline 8 & NA & NA & 39.7 & 39.6 \\
\hline 9 & $1.89(\mathrm{bq}, 7.0 \mathrm{~Hz})$ & $1.90(\mathrm{bq}, 7.0 \mathrm{~Hz})$ & 44.4 & 44.4 \\
\hline 10 & NA & NA & 140.3 & 140.2 \\
\hline 11 & NA & NA & 175.4 & 175.3 \\
\hline $12 \mathrm{a}$ & $2.55(\mathrm{dd}, 8.9,17.2 \mathrm{~Hz})$ & $2.56(\mathrm{dd}, 9.0,17.3 \mathrm{~Hz})$ & 30.2 & 30.2 \\
\hline $12 b$ & $2.74(\mathrm{dd}, 10.4,17.2 \mathrm{~Hz})$ & $2.74,(\mathrm{dd}, 10.4,17.3 \mathrm{~Hz})$ & 30.2 & 30.2 \\
\hline 13 & $3.06-3.00(\mathrm{~m})$ & $3.04(\mathrm{~m})$ & 42.1 & 42.1 \\
\hline 14 & $2.81(\mathrm{t}, 6.7 \mathrm{~Hz})$ & $2.81(\mathrm{t}, 6.6 \mathrm{~Hz})$ & 51.7 & 51.7 \\
\hline 15 & $6.53(\mathrm{~d}, 7.3 \mathrm{~Hz})$ & $6.52(\mathrm{~d}, 6.6 \mathrm{~Hz})$ & 104.8 & 104.8 \\
\hline 16 & $6.04(\mathrm{~d}, 4.4 \mathrm{~Hz})$ & $6.04(\mathrm{~d}, 4.0 \mathrm{~Hz})$ & 96.1 & 96.0 \\
\hline 17 & $0.82(\mathrm{~s})$ & $0.82(\mathrm{~s})$ & 18.9 & 18.9 \\
\hline 18 & $0.84(\mathrm{~s})$ & $0.84(\mathrm{~s})$ & 26.8 & 26.8 \\
\hline 19 & $0.87(\mathrm{~s})$ & Not Reported & 27.6 & 27.6 \\
\hline 20 & $1.00(\mathrm{~d}, 6.8 \mathrm{~Hz})$ & $1.01(\mathrm{~d}, 7.0 \mathrm{~Hz})$ & 11.6 & 11.6 \\
\hline 21 & NA & NA & 169.7 & 169.7 \\
\hline 22 & $2.09(\mathrm{~s})$ & $2.10(\mathrm{~s})$ & 21.2 & 21.1 \\
\hline
\end{tabular}


Table S4: Comparison of ${ }^{13} \mathrm{C}$ NMR of Natural and Synthetic Dendrillolide A.

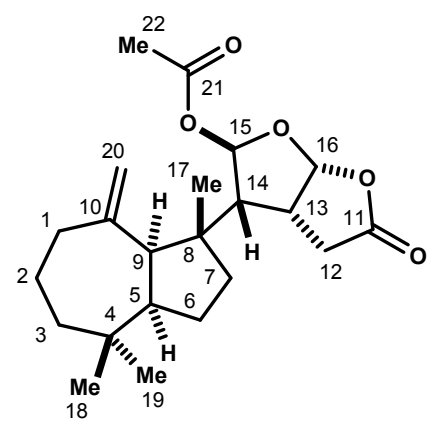

dendrillolide $A$

${ }^{13}$ C NMR (150 MHz) ${ }^{7-10} \quad$ Solvent: $\mathbf{C}_{6} \mathbf{D}_{6}$

\begin{tabular}{|c|c|c|}
\hline Atom & $\begin{array}{c}{ }^{13} \text { C Shift } \\
\text { Exp. }\end{array}$ & $\begin{array}{c}{ }^{13} \text { C Shift } \\
\text { Lit. }^{16}\end{array}$ \\
\hline $1 \mathrm{a}$ & 28.7 & 28.7 \\
\hline $1 \mathrm{~b}$ & 28.7 & 28.7 \\
\hline $2 a$ & 38.1 & 38.8 \\
\hline $2 b$ & 38.1 & 38.8 \\
\hline $3 a$ & 37.6 & 37.7 \\
\hline $3 b$ & 37.6 & 37.7 \\
\hline 4 & 46.7 & 46.7 \\
\hline 5 & 54.9 & 54.9 \\
\hline 6 & 27.1 & 27.1 \\
\hline 7 & 37.7 & 37.7 \\
\hline 8 & 36.0 & 36.0 \\
\hline 9 & 55.7 & 55.7 \\
\hline 10 & 153.8 & 153.7 \\
\hline 11 & 174.8 & 175.2 \\
\hline $12 \mathrm{a}$ & 28.8 & 28.8 \\
\hline $12 b$ & 28.8 & 28.8 \\
\hline 13 & 41.9 & 41.9 \\
\hline 14 & 54.5 & 54.5 \\
\hline 15 & 105.0 & 105.0 \\
\hline 16 & 97.1 & 97.1 \\
\hline 17 & 24.1 & 24.1 \\
\hline 18 & 25.7 & 25.8 \\
\hline 19 & 34.5 & 34.5 \\
\hline $20 \mathrm{a}$ & 114.3 & 113.3 \\
\hline $20 b$ & 114.3 & 113.3 \\
\hline 21 & 169.0 & 169.1 \\
\hline 22 & 20.7 & 20.7 \\
\hline
\end{tabular}




\section{ORTEP Image of Mukaiyama-Hydration Product 32}

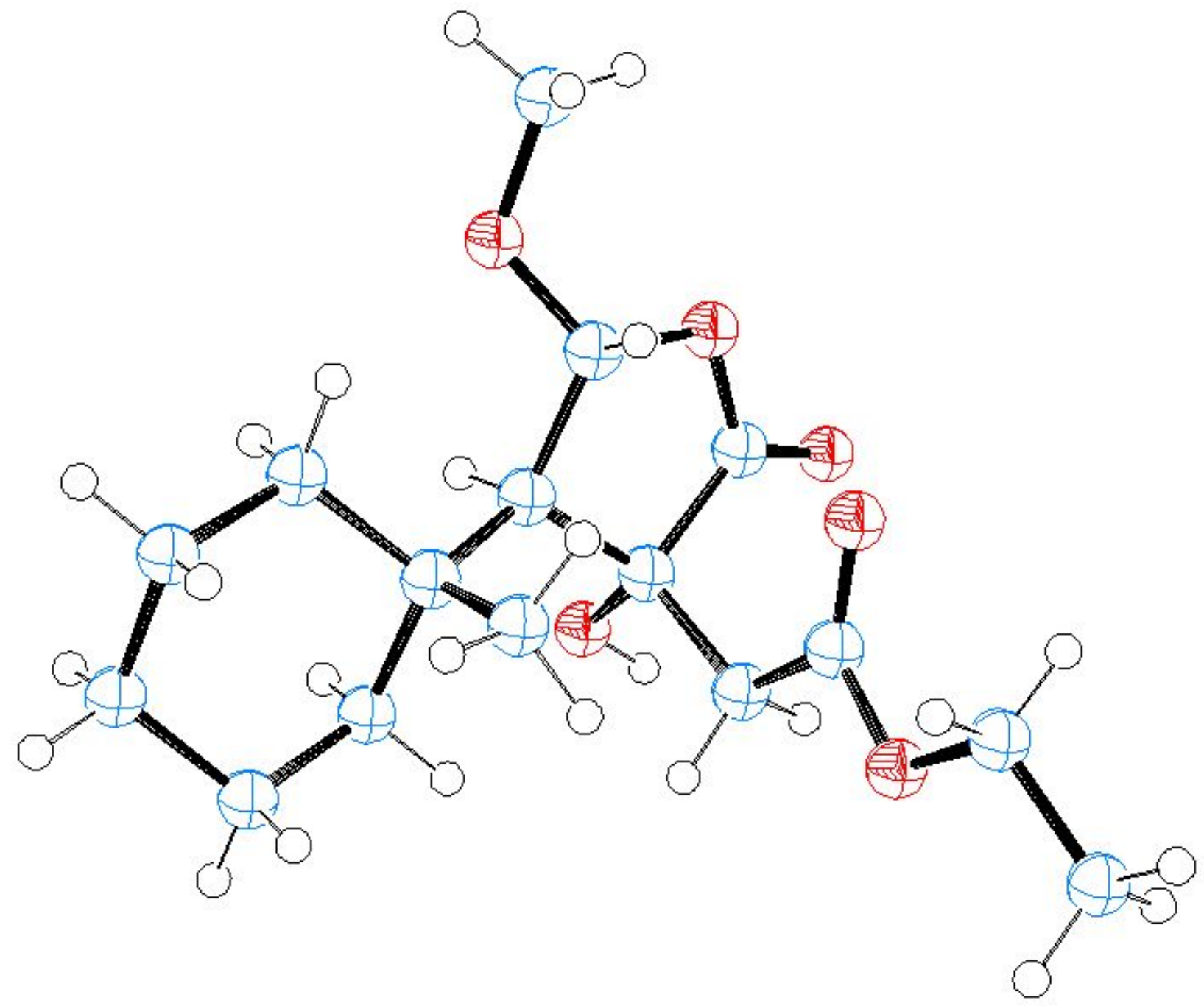

Displacement ellipsoids are drawn at the $50 \%$ probability level and $\mathrm{H}$ atoms are shown as small spheres of arbitrary radii; Black $=$ carbon, Red $=$ oxygen .

Table S1. Crystal data and structure refinement for Compound 32.

Identification code

Empirical formula

Formula weight

Temperature

Wavelength

Crystal system

Space group

Unit cell dimensions leo298

$\mathrm{C}_{16} \mathrm{H}_{26} \mathrm{O}_{6}$

314.37

$88(2) \mathrm{K}$

$0.71073 \AA$

Triclinic

$P \overline{1}$

$\mathrm{a}=9.0979(11) \AA \quad \alpha=97.5790(15)^{\circ}$.

$\mathrm{b}=9.8102(12) \AA \quad \beta=99.4092(15)^{\circ}$. 


$$
\mathrm{c}=18.733(2) \AA \quad \gamma=92.7169(16)^{\circ} .
$$

Volume

Density (calculated)

Absorption coefficient

$\mathrm{F}(000)$

Crystal color

Crystal size

Theta range for data collection

Index ranges

Reflections collected

Independent reflections

Completeness to theta $=25.500^{\circ}$

Absorption correction

Max. and min. transmission

Refinement method

Data / restraints / parameters

Goodness-of-fit on $\mathrm{F}^{2}$

Final $\mathrm{R}$ indices $[\mathrm{I}>2 \operatorname{sigma}(\mathrm{I})=6267$ data $]$

$\mathrm{R}$ indices (all data, $0.73 \AA$ )

Largest diff. peak and hole
$1631.0(3) \AA^{3}$

4

$1.280 \mathrm{Mg} / \mathrm{m}^{3}$

$0.097 \mathrm{~mm}^{-1}$

680

colorless

$0.551 \times 0.388 \times 0.329 \mathrm{~mm}^{3}$

2.099 to $29.010^{\circ}$

$-11 \leq h \leq 12,-13 \leq k \leq 13,-25 \leq l \leq 25$

18926

$7953[\mathrm{R}(\mathrm{int})=0.0243]$

$99.6 \%$

Semi-empirical from equivalents

0.8621 and 0.7658

Full-matrix least-squares on $\mathrm{F}^{2}$

7953 / 0 / 605

1.036

$\mathrm{R} 1=0.0394, \mathrm{wR} 2=0.1029$

$\mathrm{R} 1=0.0526, \mathrm{wR} 2=0.1136$

0.411 and -0.272 e. $\AA^{-3}$ 


\section{References:}

1. Obradors, C.; Martinez, R. M.; Shenvi, R. A. Ph(i-PrO) $\mathrm{SiH}_{2}$ : An exceptional reductant for metal-catalyzed hydrogen atom transfers. J. Am. Chem. Soc. 2016, 138, 4962-4971.

2. Chung, S. K. Selective reduction of mono- and disubstituted olefins by sodium borohydride and cobalt(II). $J$. Org. Chem. 1979, 44, $1014-1016$.

3. Ma, X.; Herzon, S.B. Non-classical selectivities in the reduction of alkenes by cobalt-mediated hydrogen atom transfer. Chem. Sci. 2015, 6, 6250-6255.

4. Iwasaki, K.; Wan, K. K.; Oppedisano, A.; Crossley, S. W. M.; Shenvi, R. A. J. Am. Chem. Soc. 2014, 136, $1300-1303$.

5. Magnus, P.; Waring, M. J.; Scott, D. A. Conjugate reduction of $\alpha, \beta$-unsaturated ketones using an MnIII catalyst, phenylsilane and isopropyl alcohol. Tetrahedron Lett. 2000, 41, 9731 - 9733.

6. Molinski, T. F.; Faulkner, D. J.; He, C.; Van Duyne, G. D.; Clardy, J. Three new rearranged spongian diterpenes from chromodoris macfarland: reappraisal of the structures of dendrillolides A and B. J. Org. Chem. 1986, 51, 4564-4567.

7. Sullivan, B.; Faulkner, D. J. Metabolites of the marine sponge Dendrilla sp. J. Org. Chem. 1984, 49, 3204 3206.

8. Macfarlandin $\mathrm{C}$ and dendrillolide $\mathrm{A}$ decompose under mildly acidic conditions such as prolonged exposure to silica gel or $\mathrm{CDCl}_{3}$, resulting in the growth of an inseparable decomposition product showing a diagnostic broad multiplet at $1.25 \mathrm{ppm}\left({ }^{1} \mathrm{H} \mathrm{NMR}, \mathrm{CDCl}_{3}\right)$ and peaks at 29.7, 29.4 and $14.1 \mathrm{ppm}\left({ }^{13} \mathrm{C} \mathrm{NMR}, \mathrm{CDCl}_{3}\right)$.

9. During studies aimed at gathering optical rotation of synthetic dendrillolide $\mathrm{A}$, it was determined that the natural product readily decomposes in $\mathrm{CHCl}_{3}$. After $30 \mathrm{~min}$ at room temperature in $\mathrm{K}_{2} \mathrm{CO}_{3}$-treated $\mathrm{CHCl}_{3}$, ca. $20 \%$ decomposition observed.

10. The reported ${ }^{1} \mathrm{H}$ NMR conditions for dendrillolide $\mathrm{A}$ were $1 \% \mathrm{C}_{6} \mathrm{D}_{6}$ in $\mathrm{CCl}_{4}$. Although, we were able to observe the key shifts in this solvent system, the poor shimming quality of the acquired spectra prevents us from reporting the ${ }^{1} \mathrm{H}$ NMR comparison between synthetic and natural dendrillolide $\mathrm{A}$. 


\section{Spectral Data}




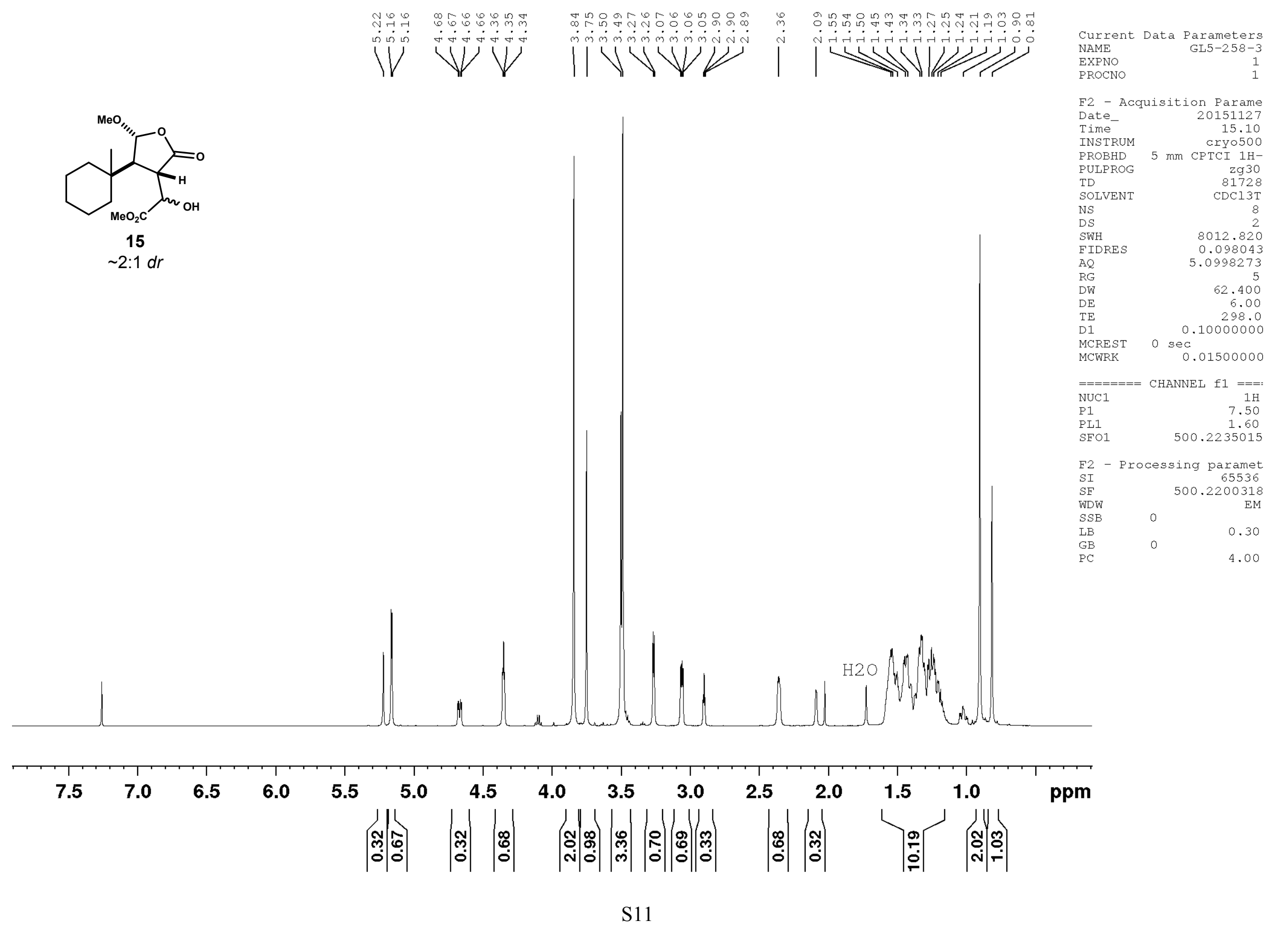




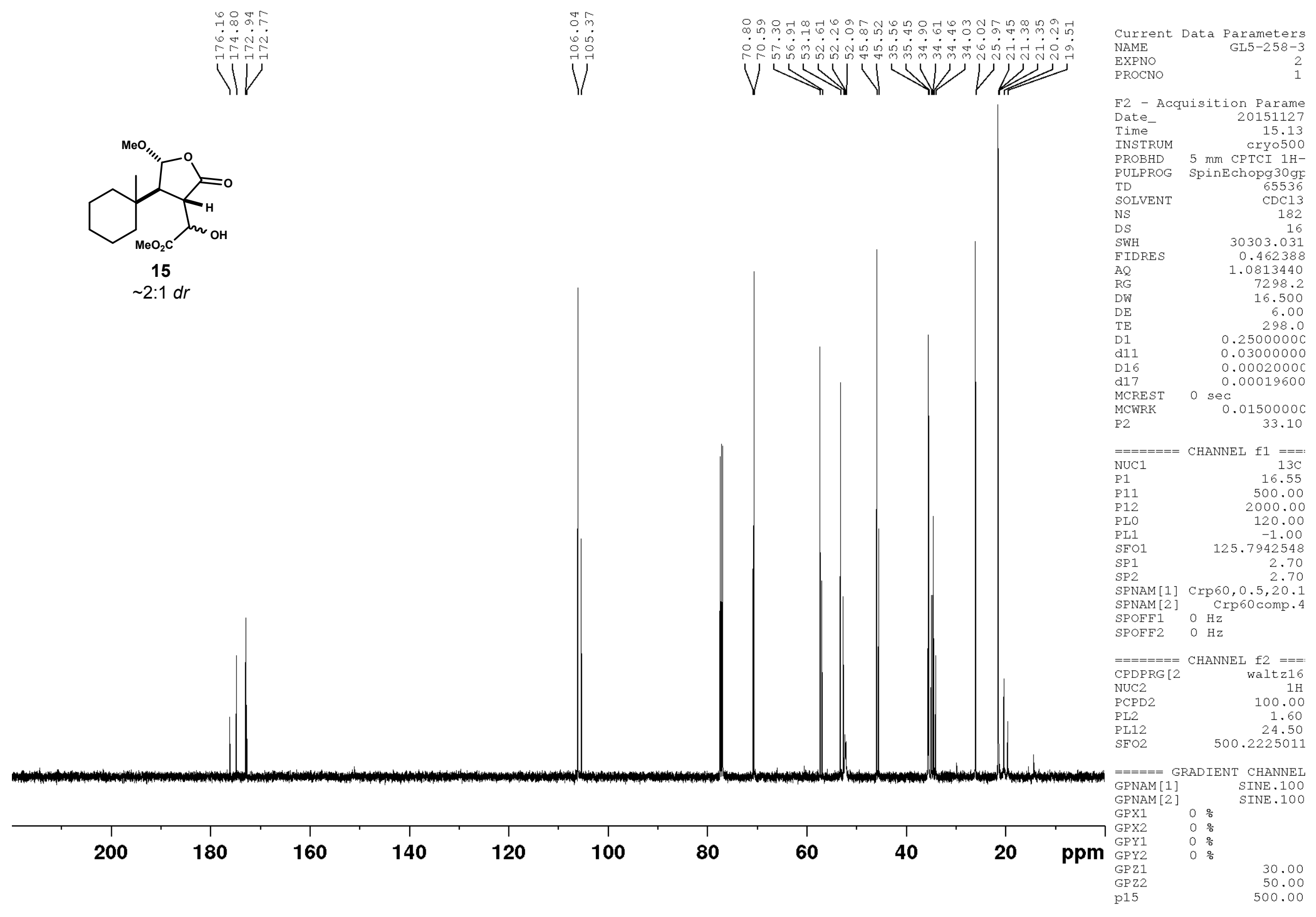


E2 - Acquisition Parane
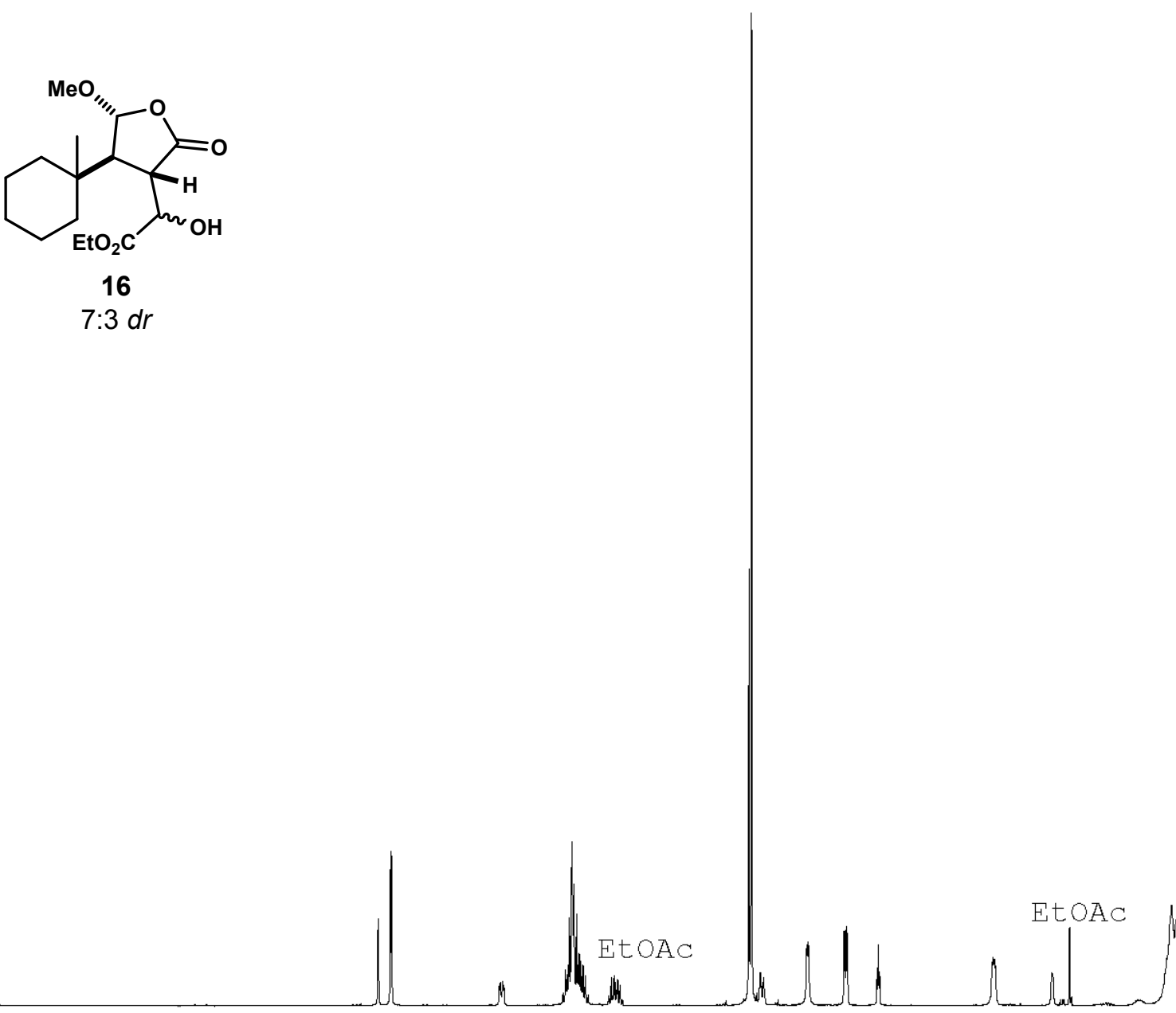

INSTRUM

PROBHD

mm $\mathrm{CPBBO} \mathrm{BB}-$

NS

SWH

FIDR

16

$7: 3 d r$
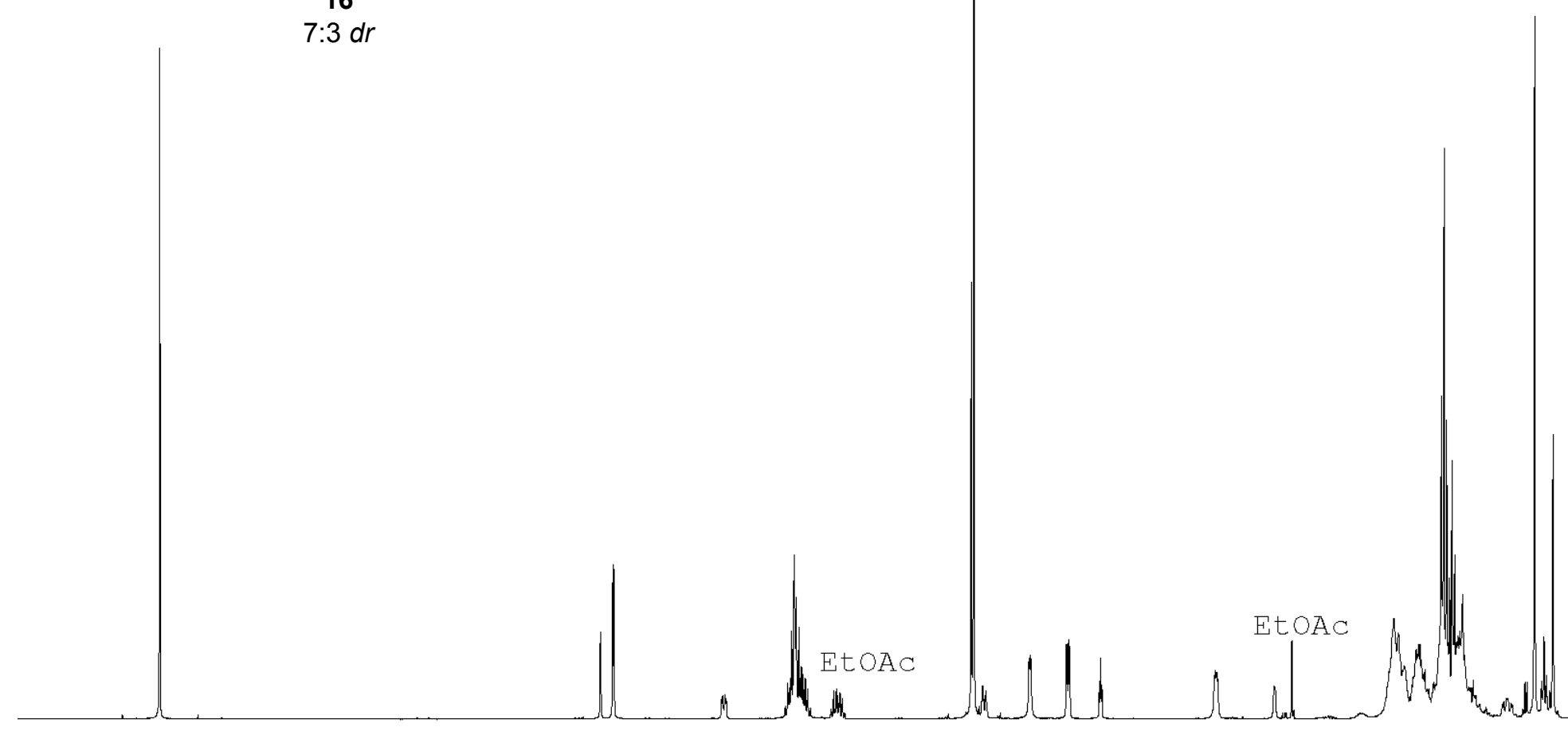

$\mathrm{AQ}$
$\mathrm{RG}$
$\mathrm{DW}$

DW

TE

D1

$====$

$\mathrm{SFO1}$

P1

98713
16

2
9615.385

5.0998478

52.000

13.81

0.10000000

$1 \mathrm{H}$
11.50

20.00000000

F2 - Processing paramet

SI 65536

600.1300340

0.30

GB

1.00

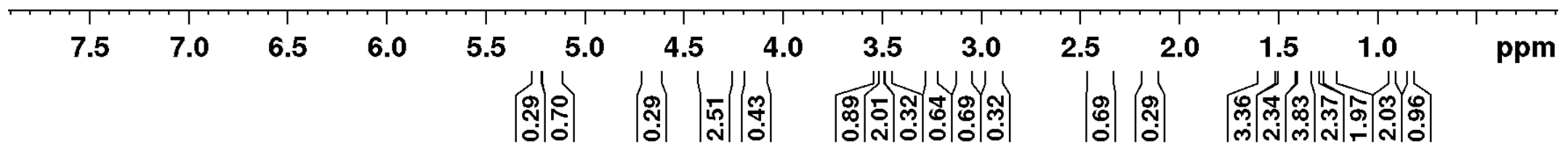




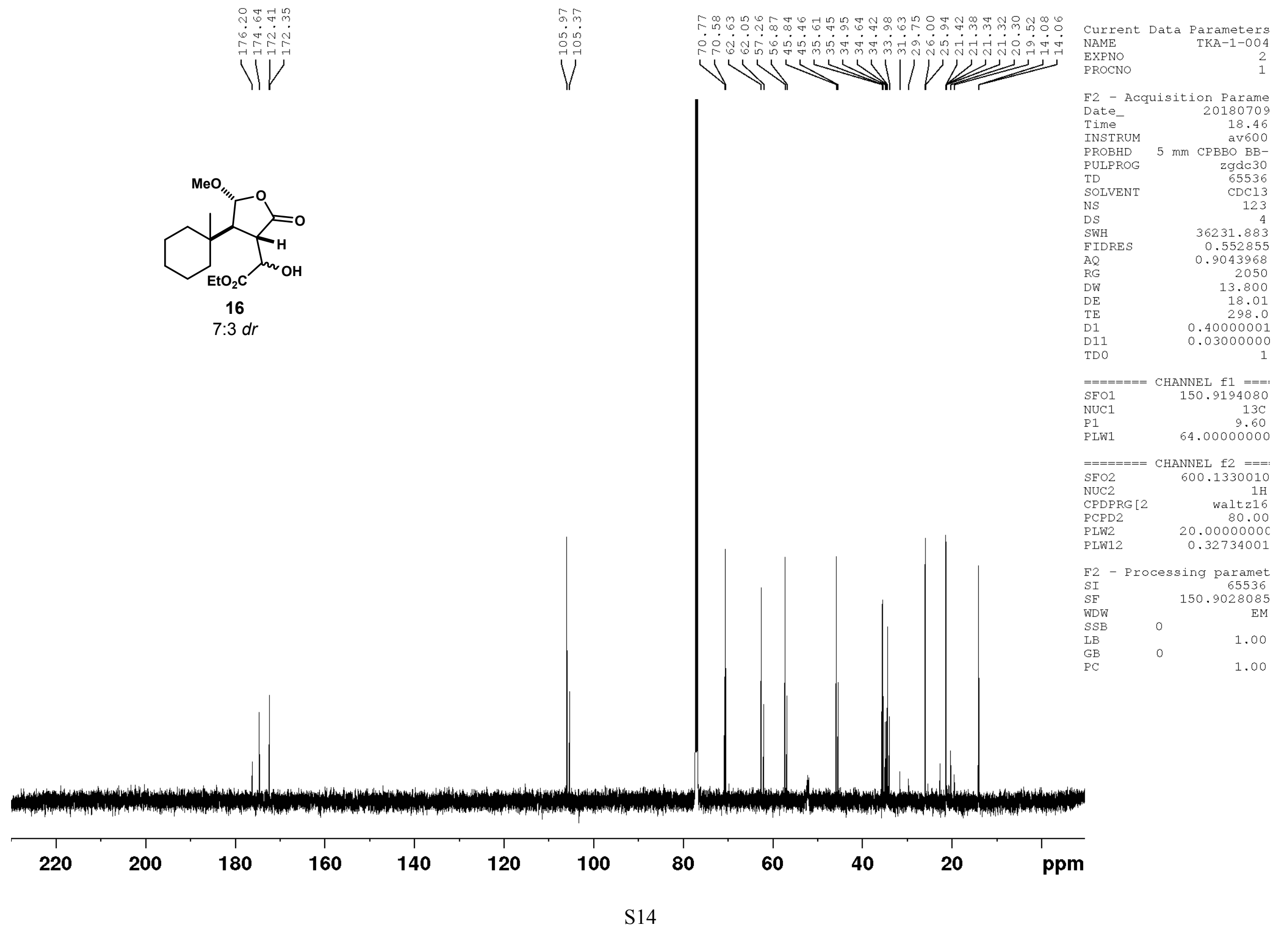




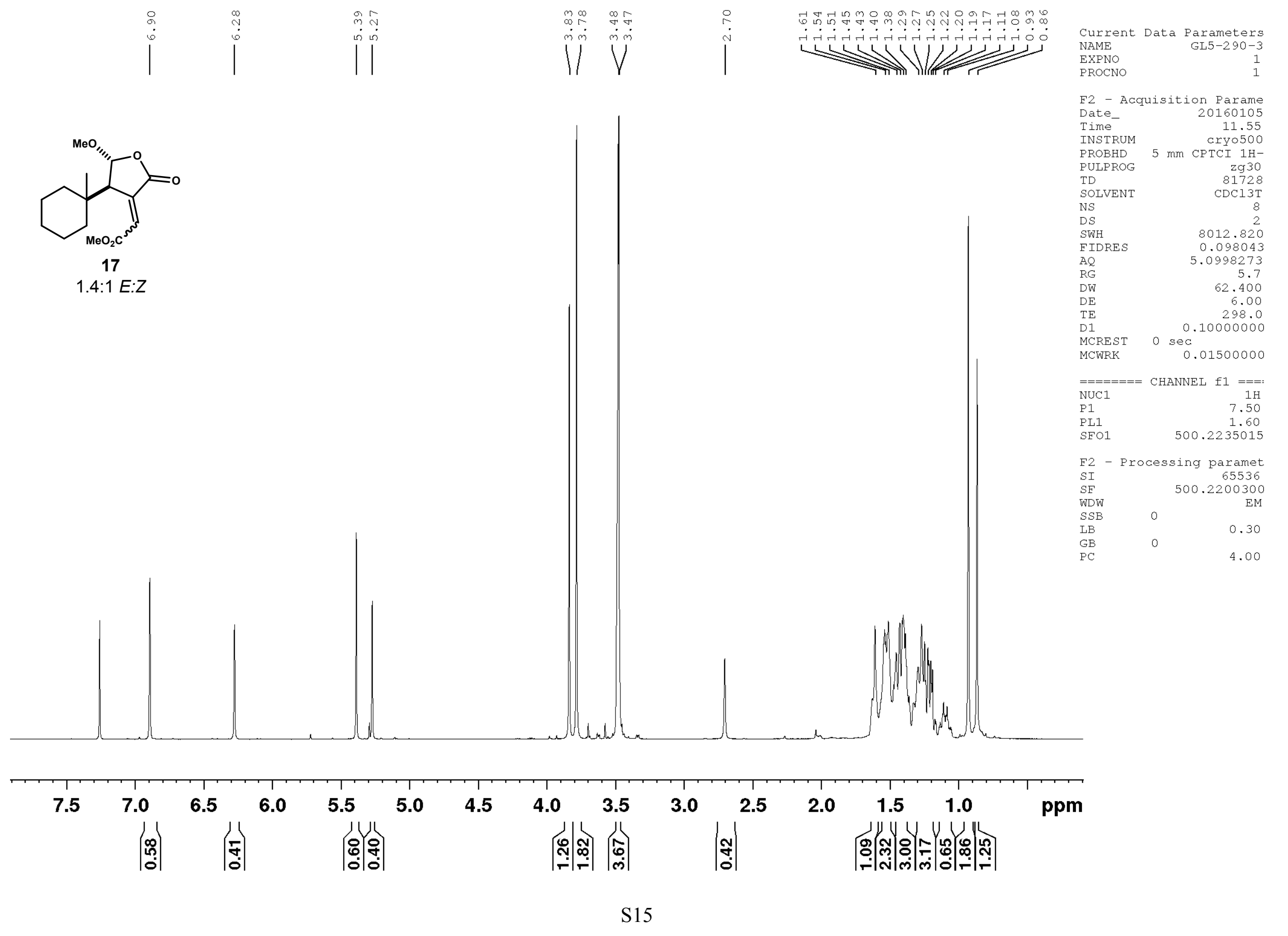




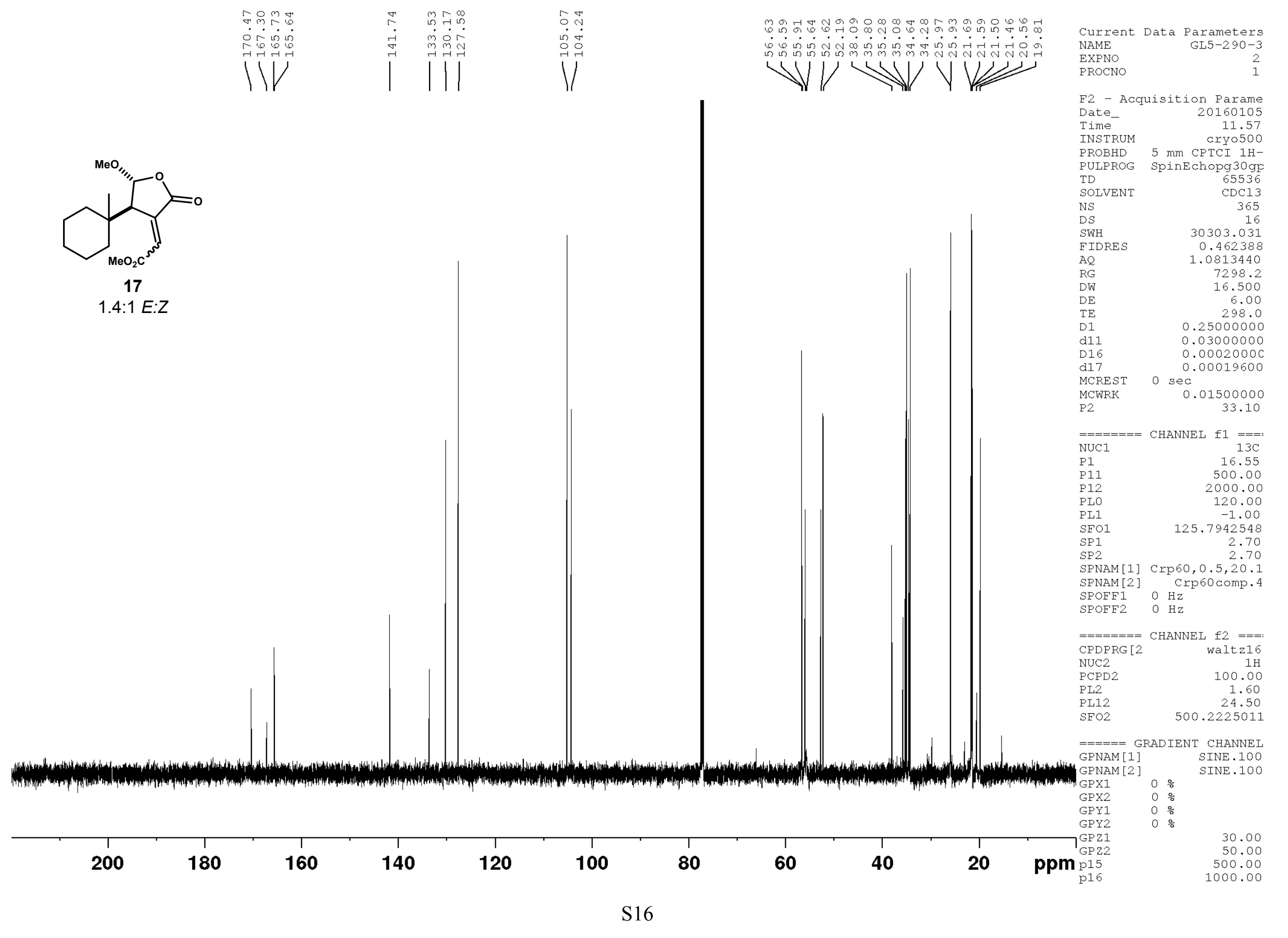




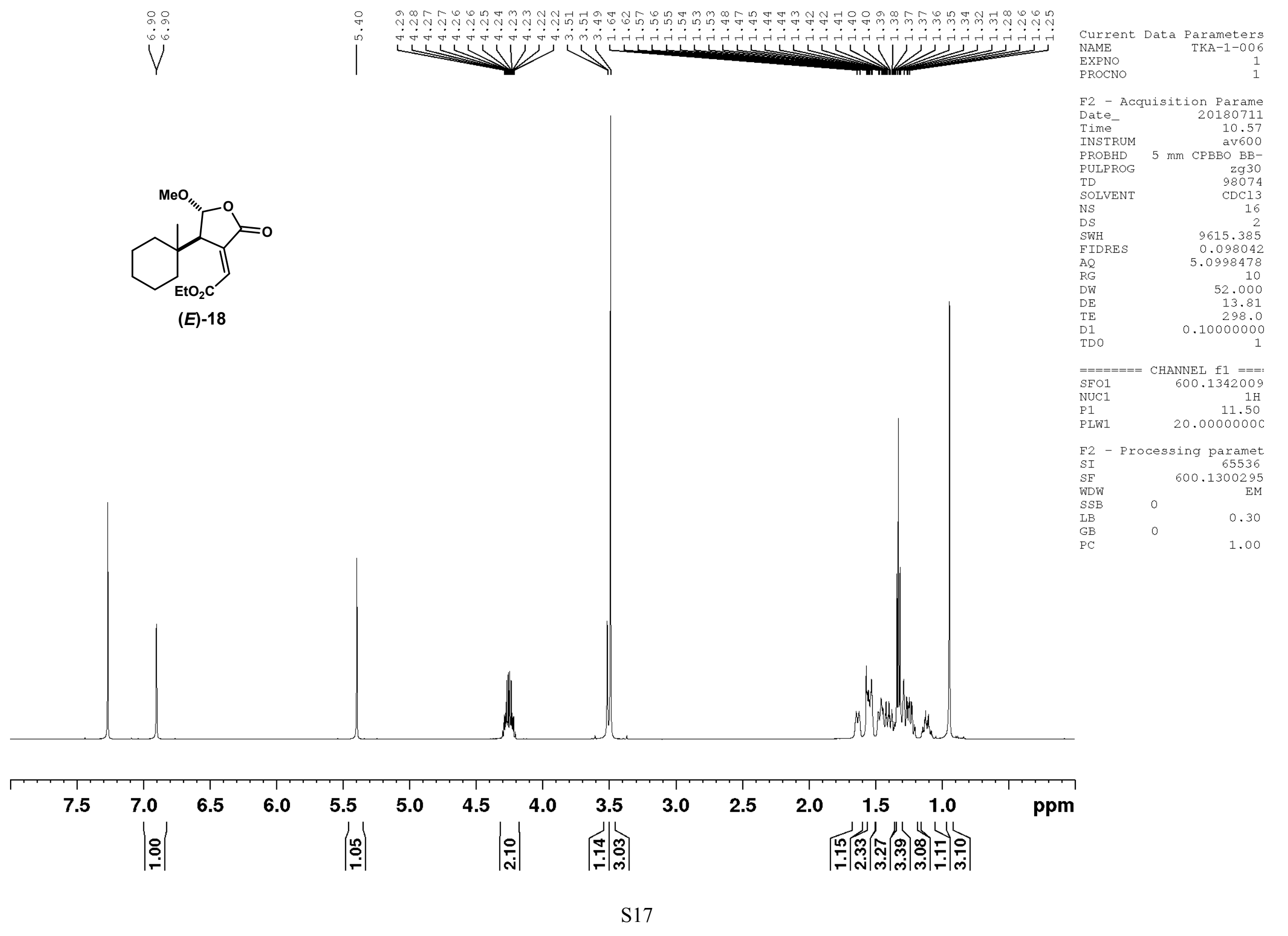




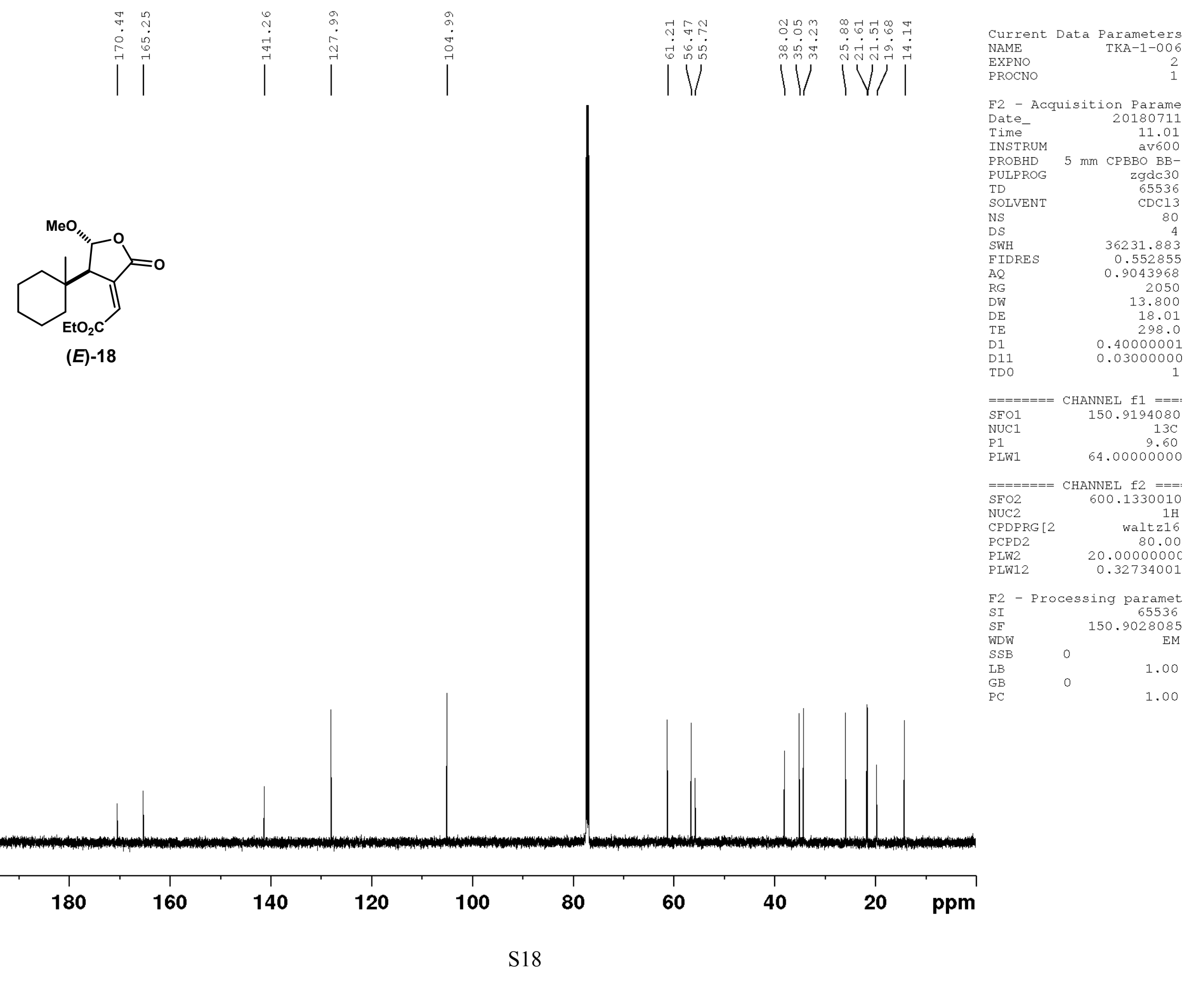




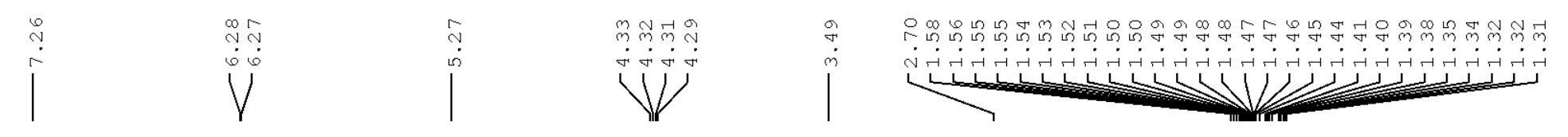

$\begin{array}{lr}\text { Current Data } & \text { Parameters } \\ \text { NAME } & \text { PZ-4061-P2 } \\ \text { EXPNO } & 1 \\ \text { PROCNO } & 1\end{array}$

F2 - Acquisition Parame Date__ 20180124 Time av600 $\mathrm{mm} \mathrm{CPBBO} \mathrm{BB}-$ PULPROG $\quad$ zg30

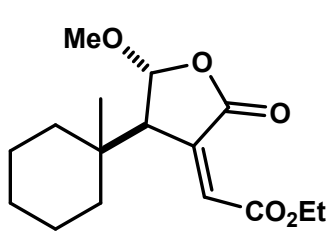

SOLVENT

NS

DS

FIDRES

AQ
RG
DW

$\mathrm{DE}$
$\mathrm{TE}$

TE

(Z)-18

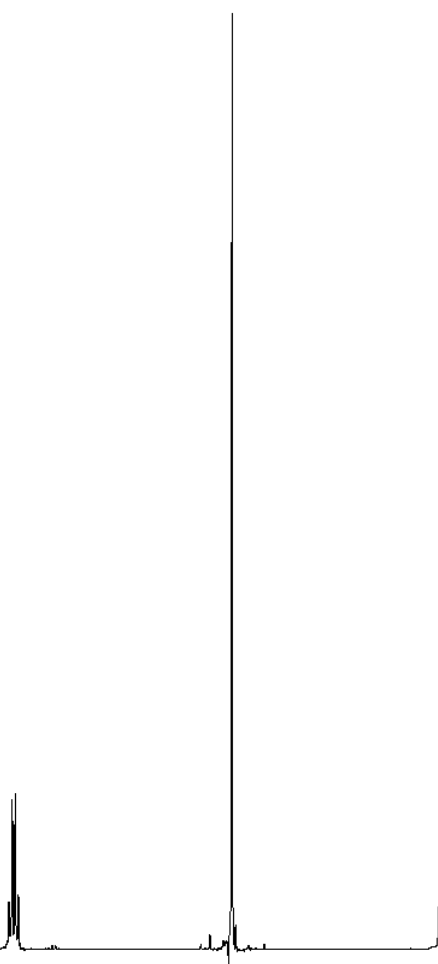

P1

600.1342009

PI

0.25

F2 - Processing

sing paramet

SF $\quad 600.1300341$

WDW

SSB

LB

$\mathrm{GB}$
$\mathrm{PC}$

0.30

1.00

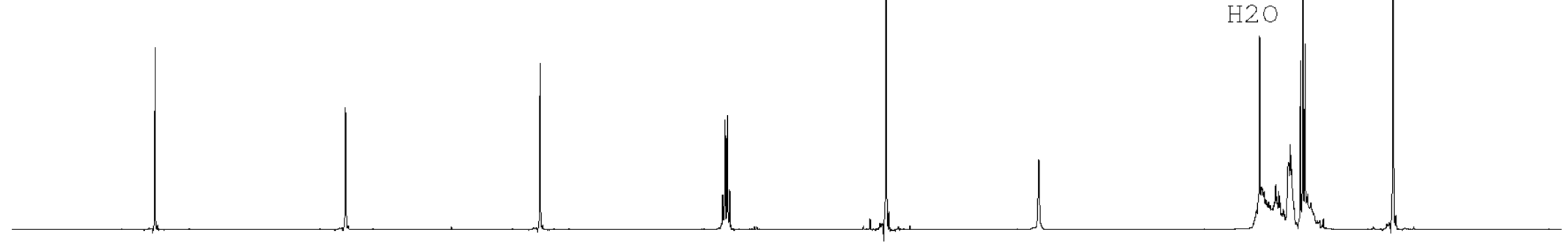

$\begin{array}{lll}7.5 & 7.0 & 6.5\end{array}$

$\left|\begin{array}{l}.5 \\ 0 \\ \hdashline\end{array}\right|$

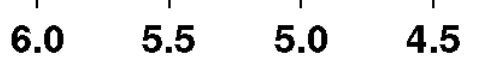

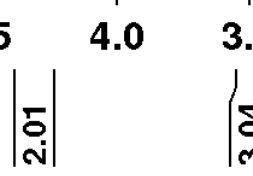

$\left|\begin{array}{l}3.5 \\ \text { ले }\end{array}\right|$

3.0

2.5

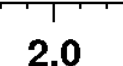

1.5

$\left|\begin{array}{c}0 \\ \infty\end{array}\right| \begin{aligned} & n \\ & \dot{\infty}\end{aligned}|\quad| \begin{gathered}\infty \\ 0 \\ \dot{m}\end{gathered} \mid$

ppm

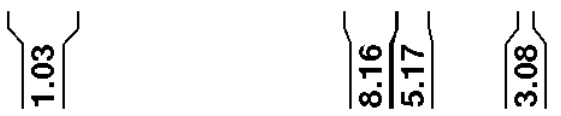




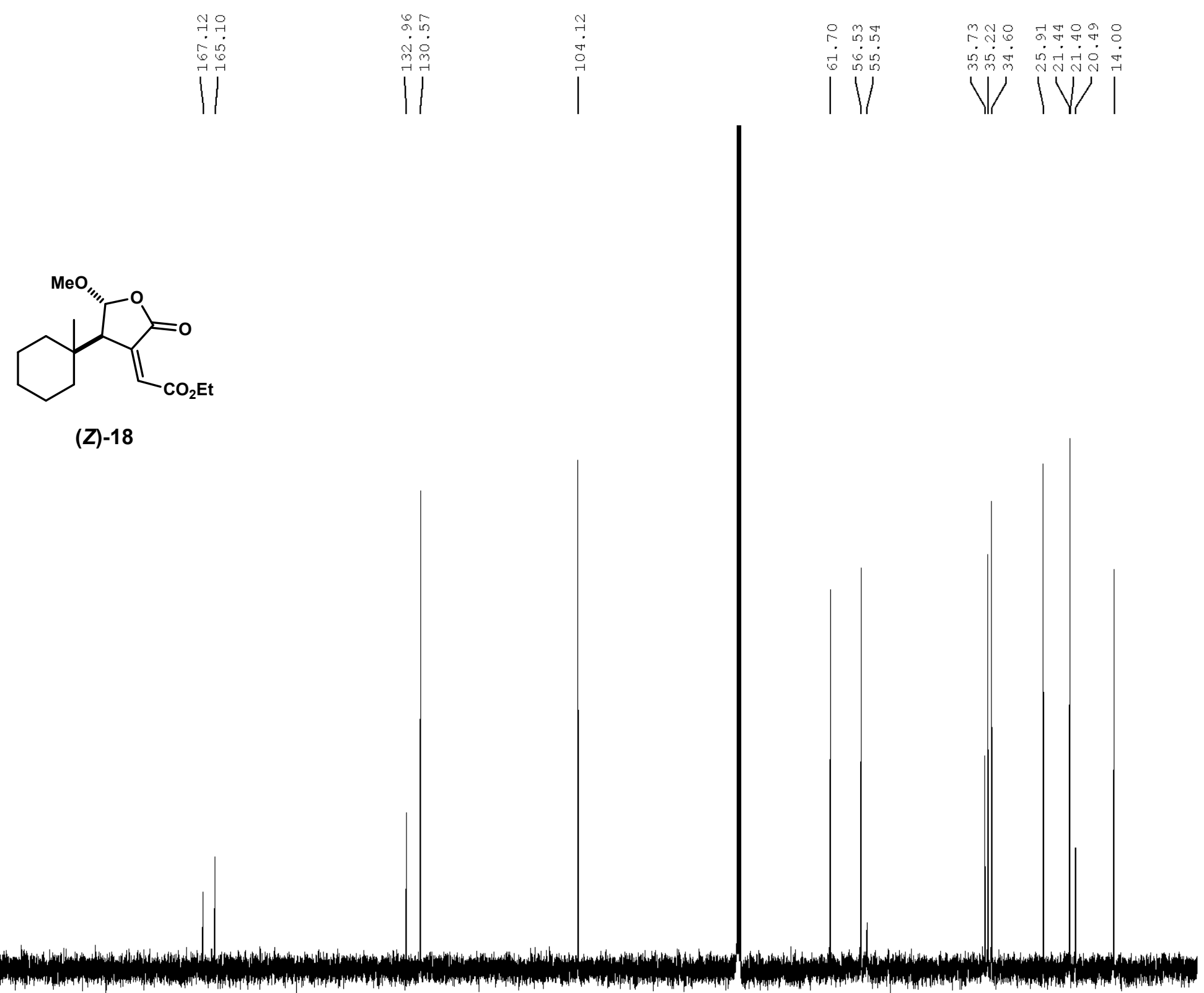

Current Data Pa

NAME

EXPNO

PROCNO

Datequisition Parame

Date-

INSTRUM

PUTPROG 5 mn CPTCI $1 \mathrm{H}-$

TD

SOLVENT

NS

DS

16
30303.031

$\begin{array}{ll} & 0.462388\end{array}$

AQ $\quad 1.0813440$

$\begin{array}{ll}\text { RG } & 7298.2 \\ \text { DW } & 16.500\end{array}$

$\begin{array}{lr}\mathrm{DE} & 6.00 \\ \mathrm{TE} & 298.0\end{array}$

D1 $\quad 0.25000000$

d11 $\quad 0.03000000$

D16 $\quad 0.00020000$

0.00019600

MCREST 0 sec

MCWRK $\quad 0.01500000$

P2

33.10

NUC1

P1
P11

CHANNEL $\mathrm{f} 1====$
$13 \mathrm{C}$

16.55
500.00
2000.00

120.00

PL1 -1.00

$\begin{array}{ll}\mathrm{SFO} & 125.7942548\end{array}$

$\begin{array}{lr}\text { SP1 } & 2.70 \\ \text { SP2 } & 2.70\end{array}$

SPNAM [1] Crp60, 0.5,20.1 SPNAM [2] Crp60comp.4 SPOFF1 $0 \mathrm{~Hz}$

SPOFF2 O H

$====== \pm$ CHANNEL $f 2===:$
waltz16 NUC2 PCPD2 100.00

PL12

24.50 GPNAM [1] SINE. 100

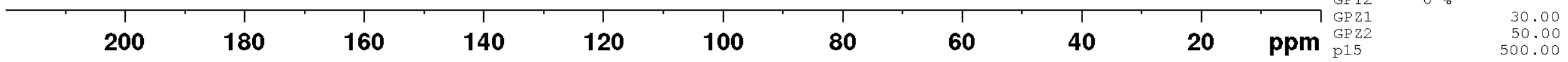




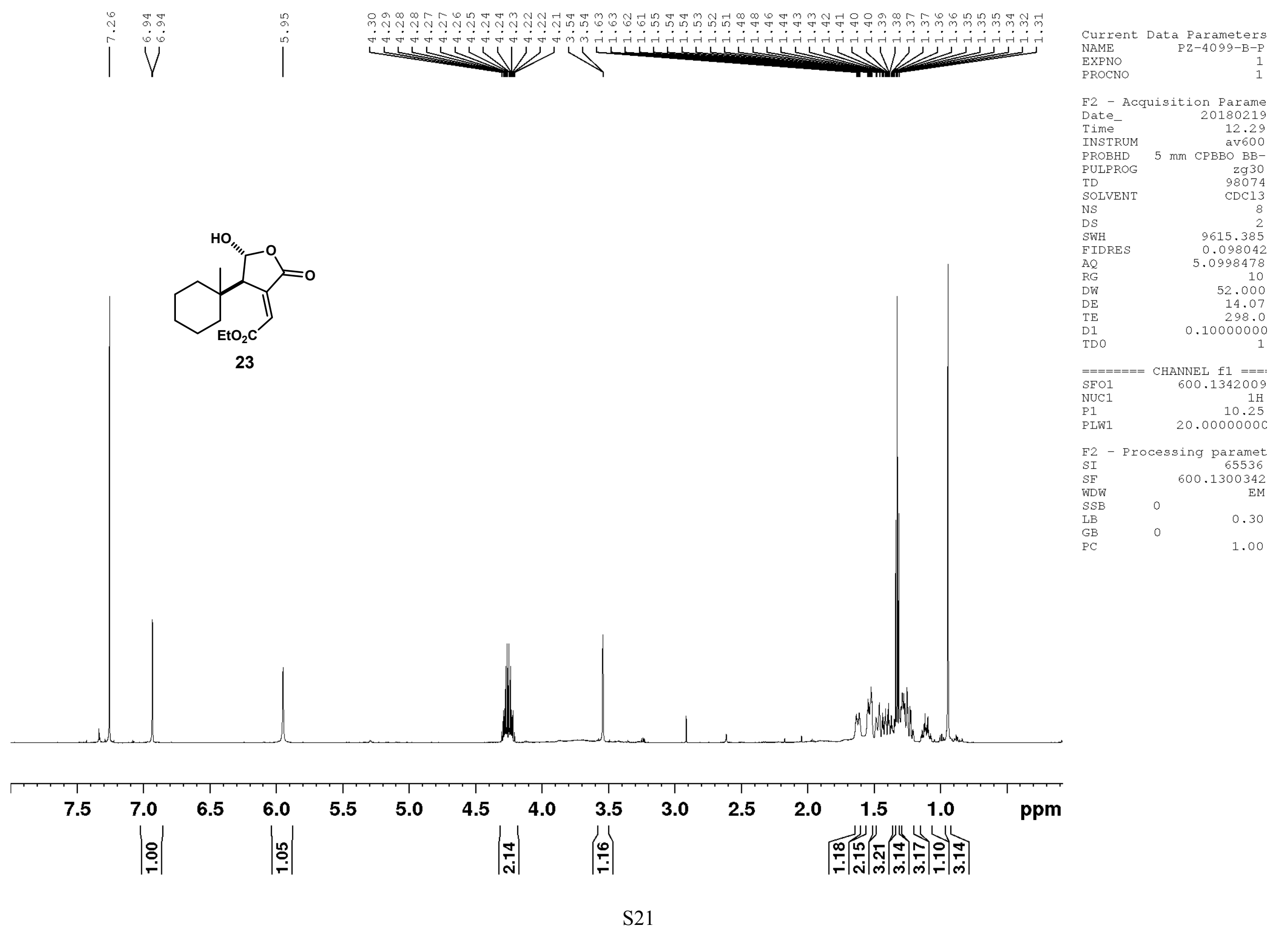




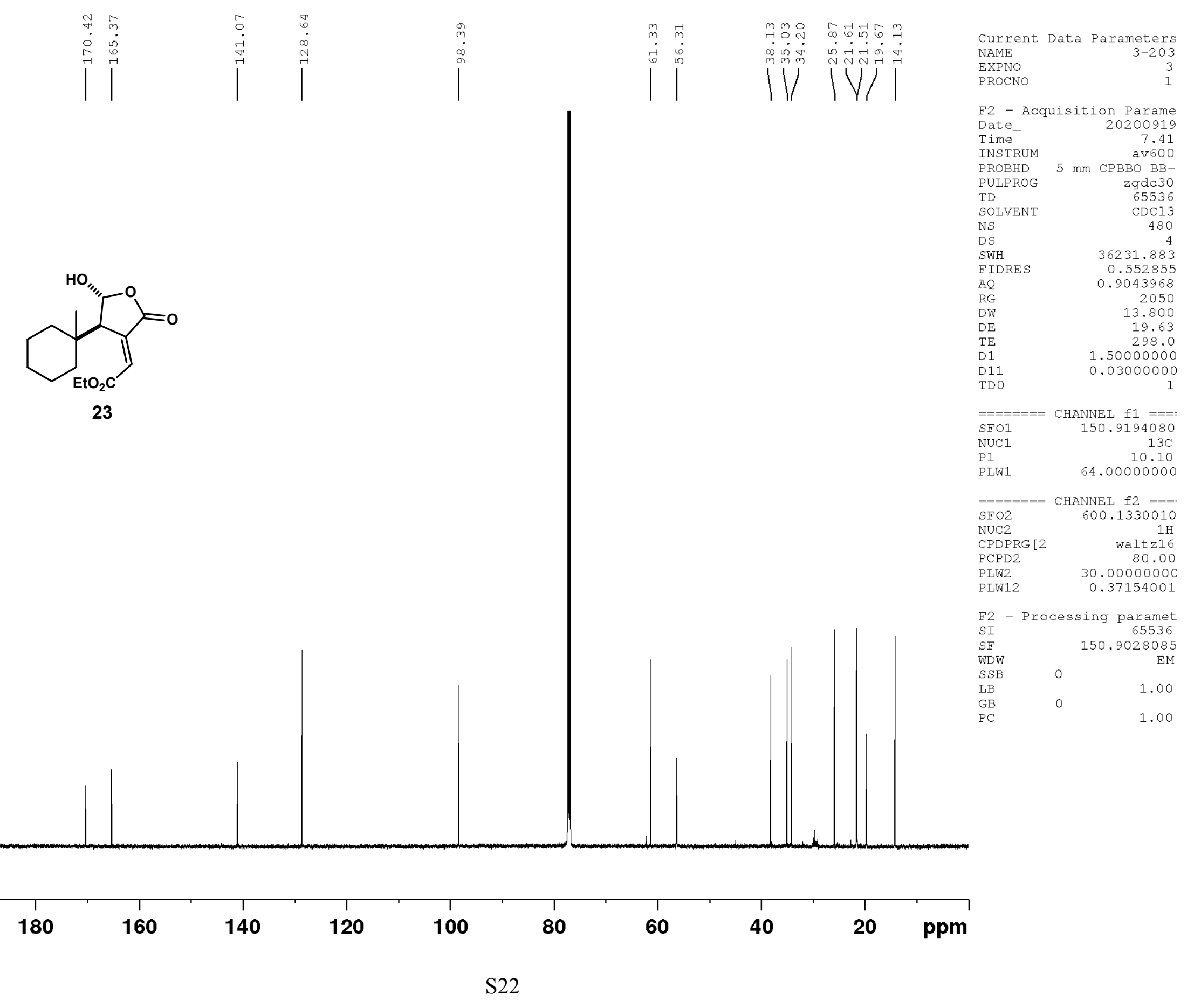




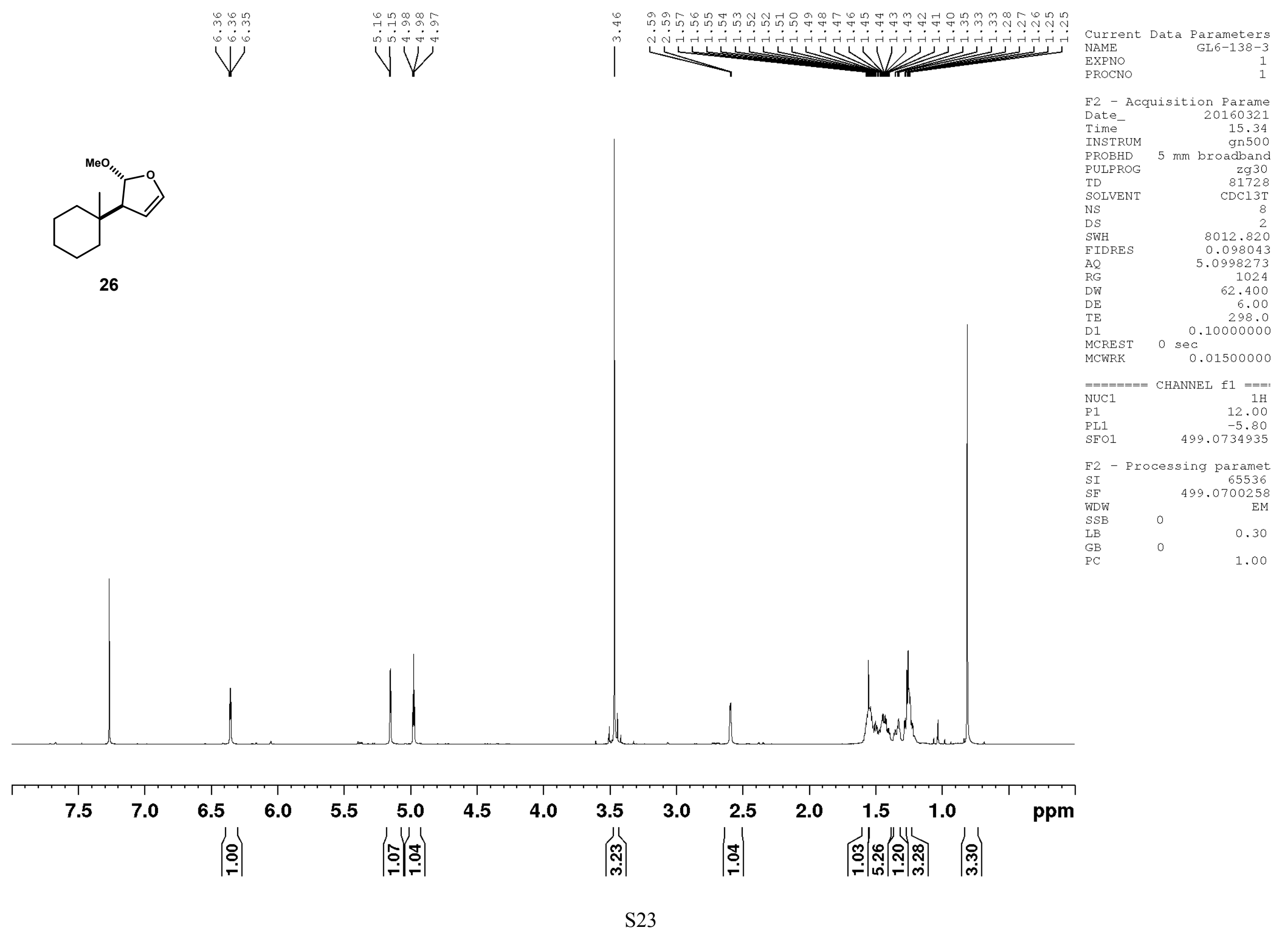




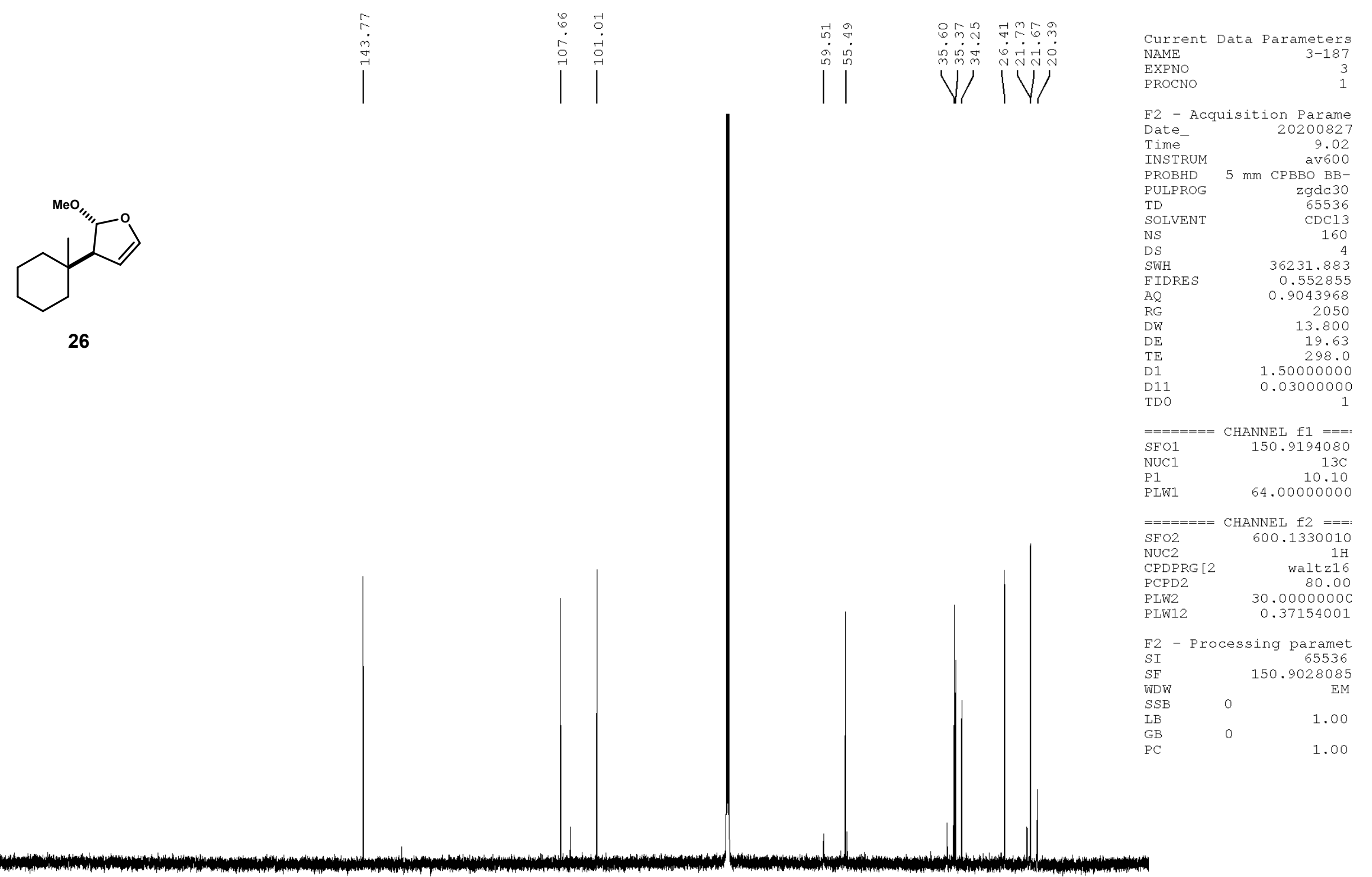




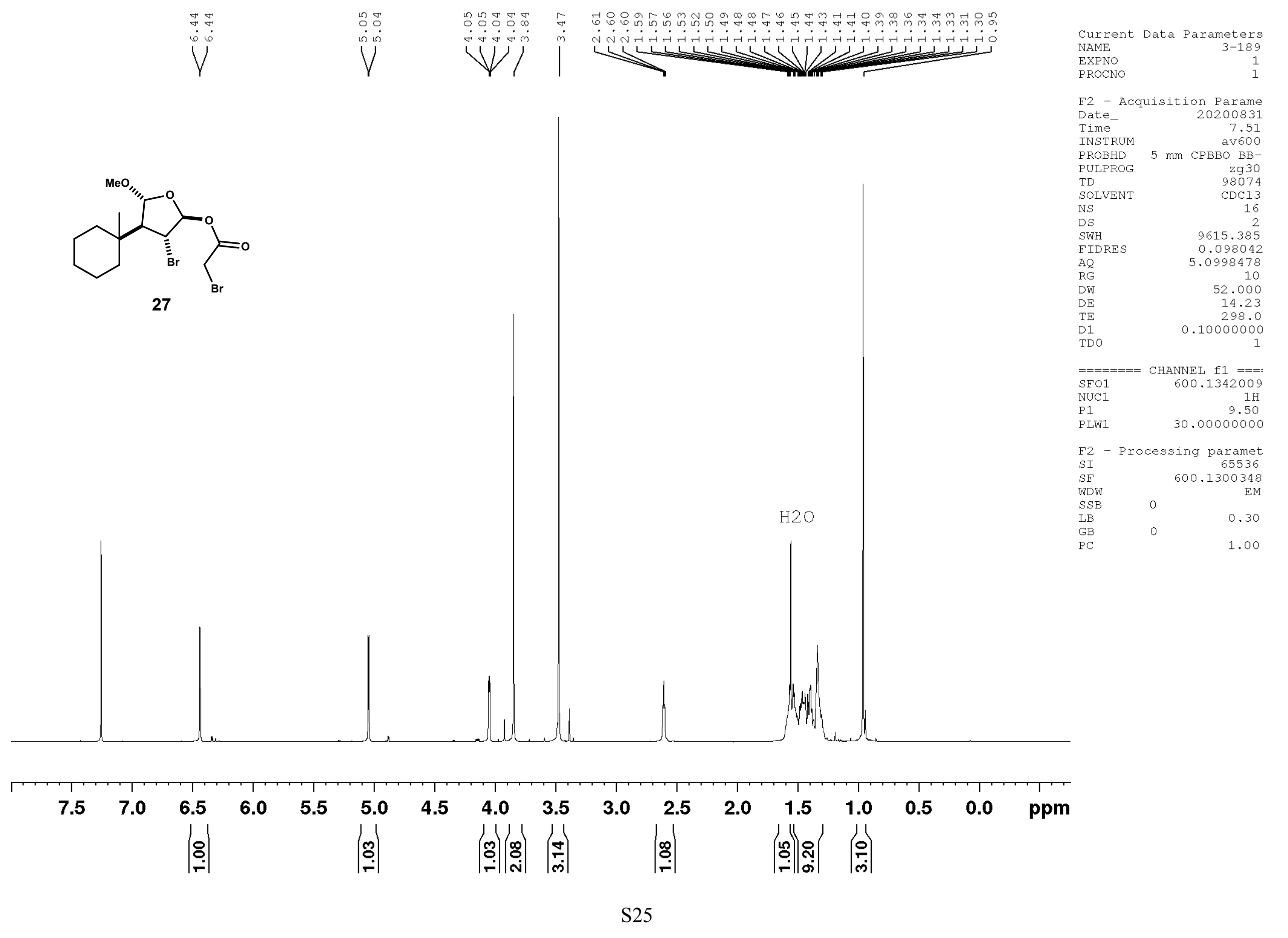




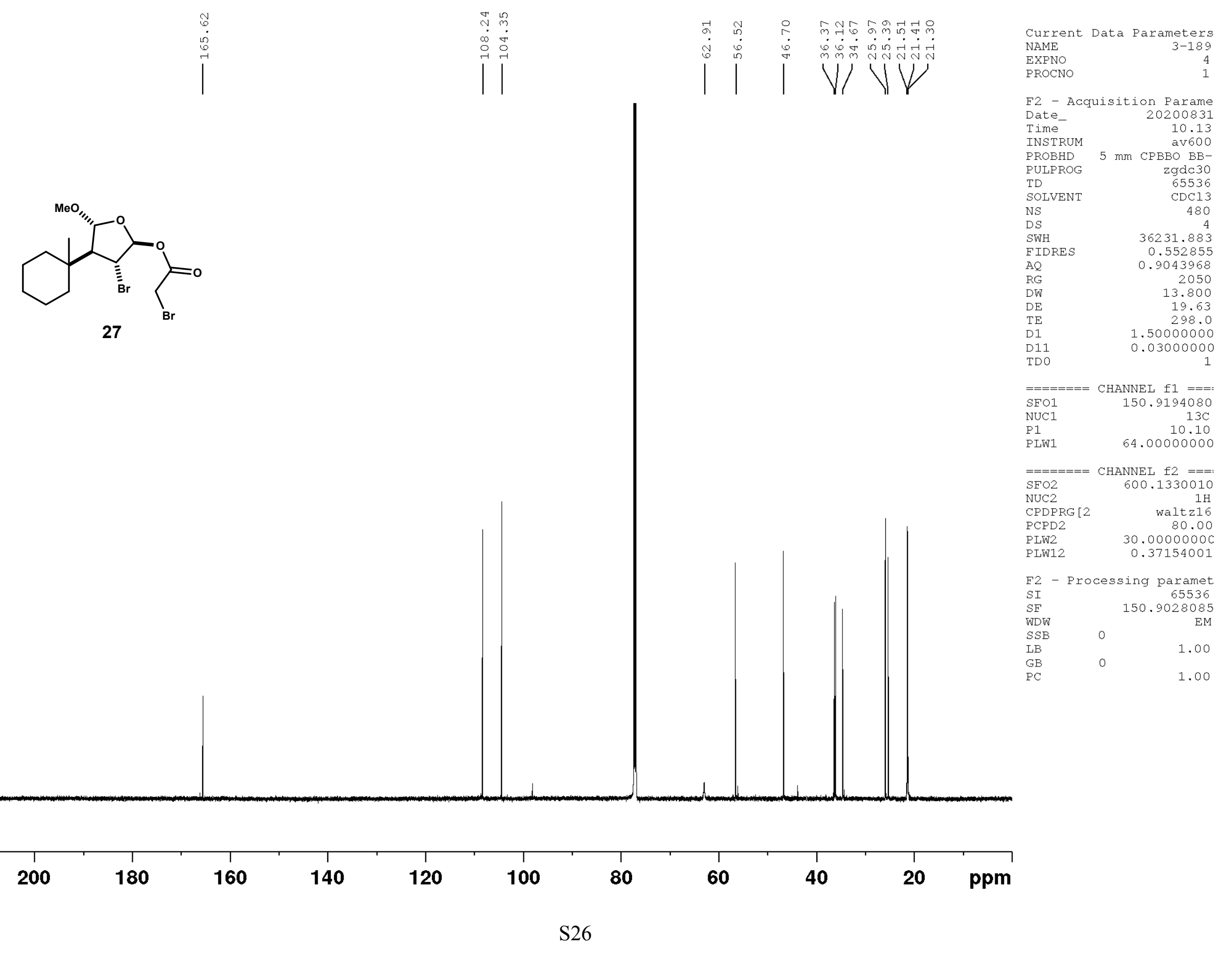




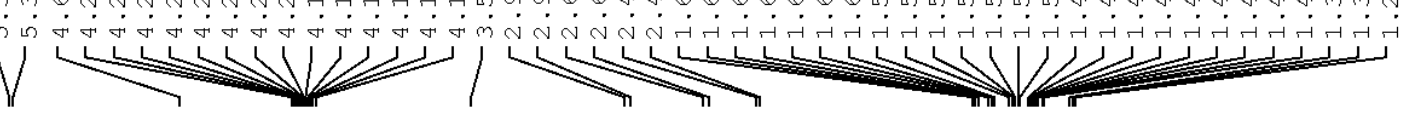

current Data Parameters NAME

PROCNO

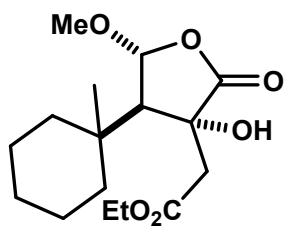

32
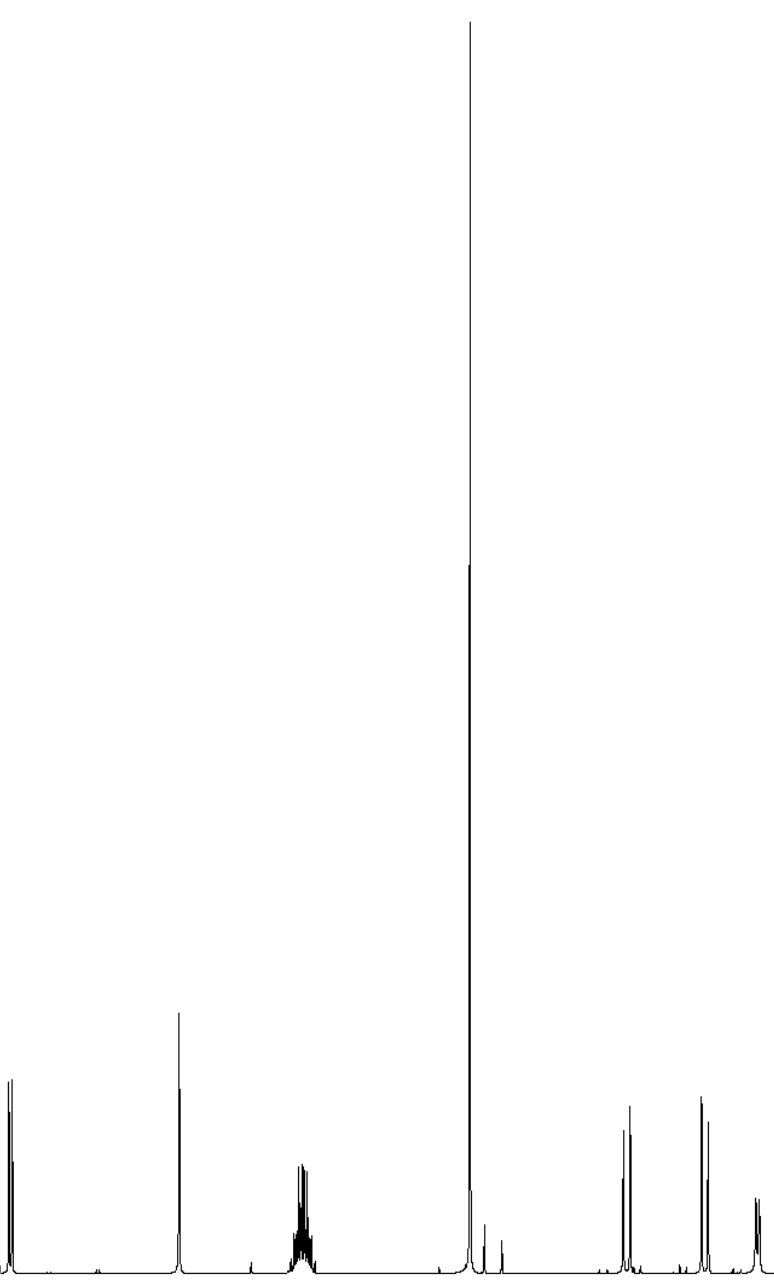

F2 - Acquisition Parame Date

Time

INSTRUM

PROBHD

12.07

$\mathrm{mm} \mathrm{CPBBO} \mathrm{BB}-$

TD

NS

DS

FIDRES $\quad 0.098042$

$\mathrm{AQ}$

$\mathrm{RG}$

DE

D1

TDO

5.0998478

10
52.000

13.70
298.0

- $=== \pm==$ CHANNEL f1 $===$

$\begin{array}{rr} & 600.1342009 \\ \text { NUC1 } & 1 \mathrm{H}\end{array}$

P1 12.00

PLW1 30.00000000

F2 - Processing paramet

SI 65536

$\begin{array}{ll}\mathrm{SF} & 600.1300341\end{array}$

SSB $\quad 0$

0.30

0.30

1.00

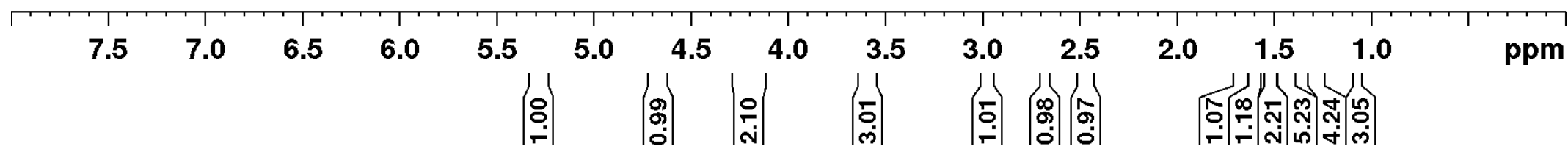



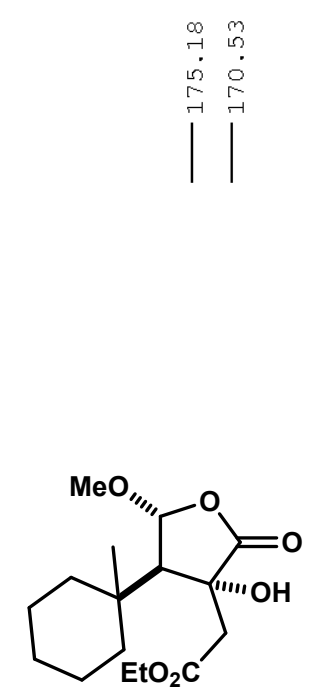

32

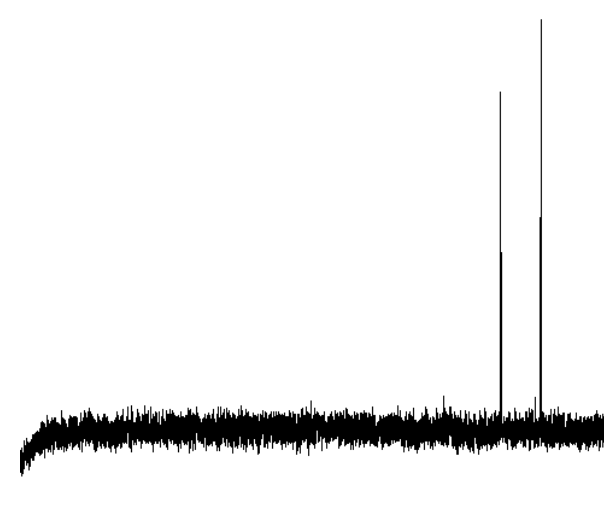

\begin{tabular}{|llllllllllll}
220 & 200 & 180 & 160 & 140 & 120 & 100 & 80 & 60 & 40 & 20 & ppm
\end{tabular}

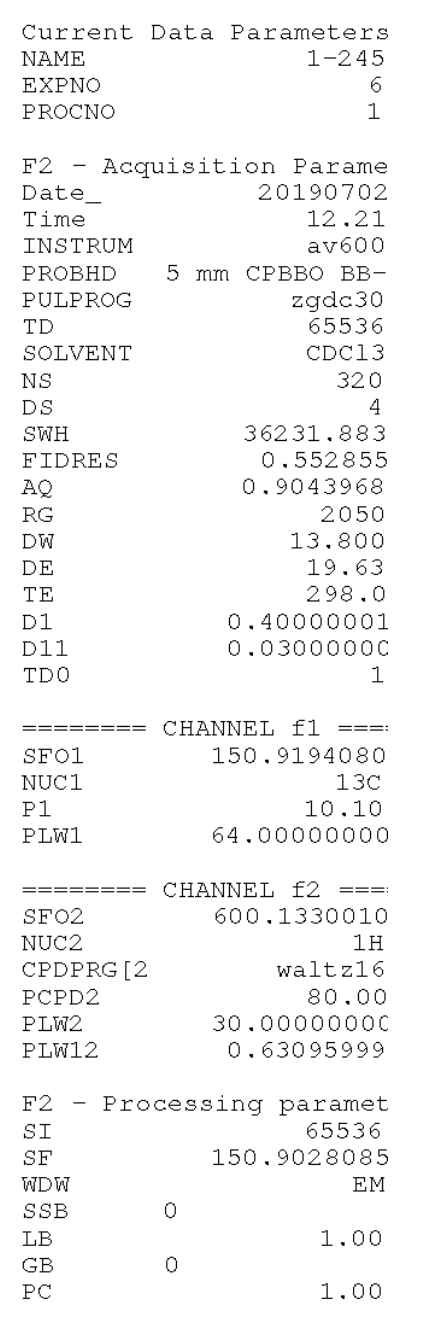


F2 - Acquisition Parame Time 20190706

$=======$ CHANNEL $\mathrm{f} 1 \mathrm{=}==\mathrm{=}$
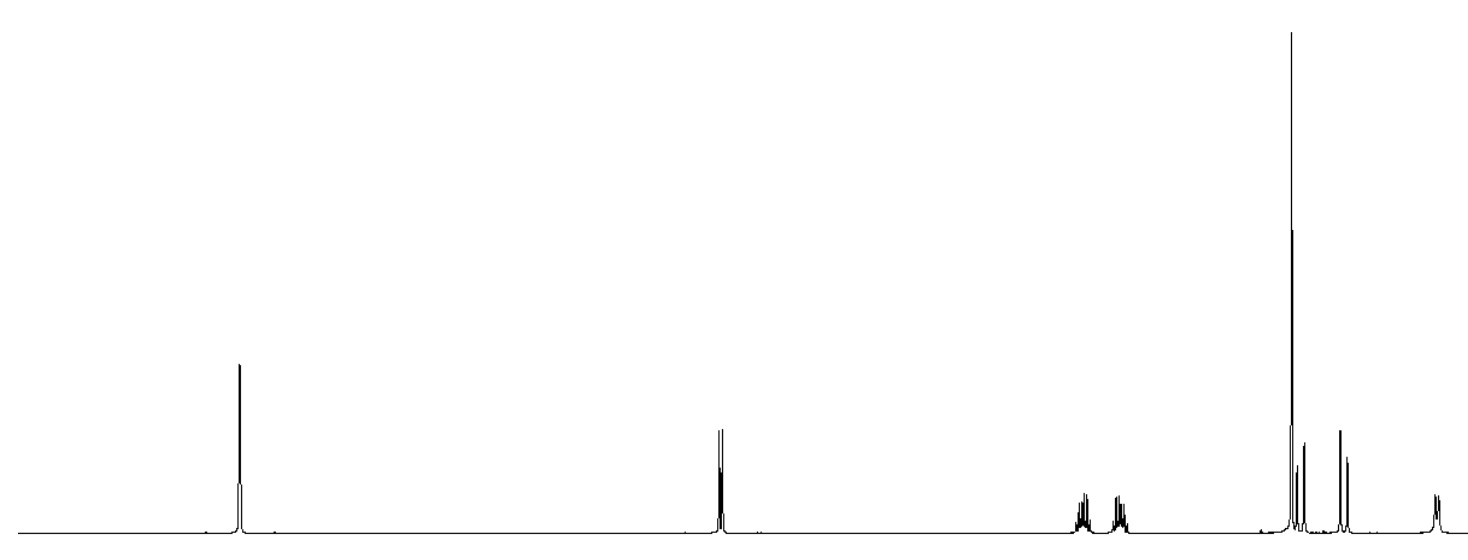

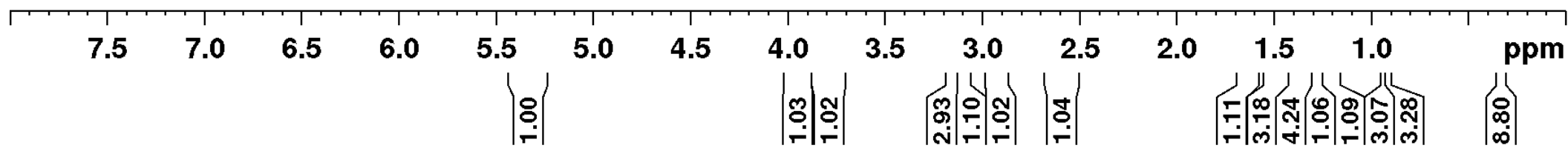




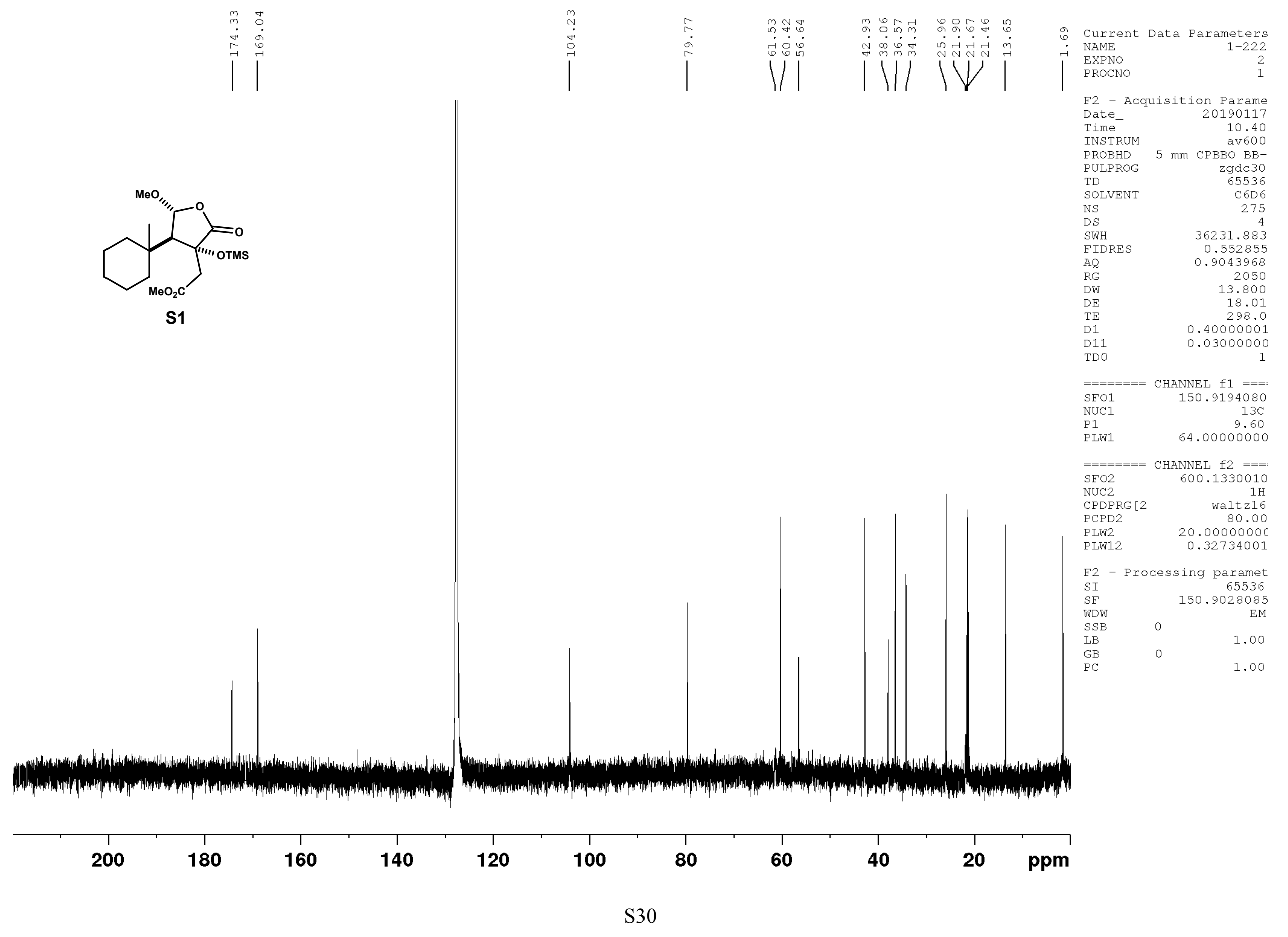




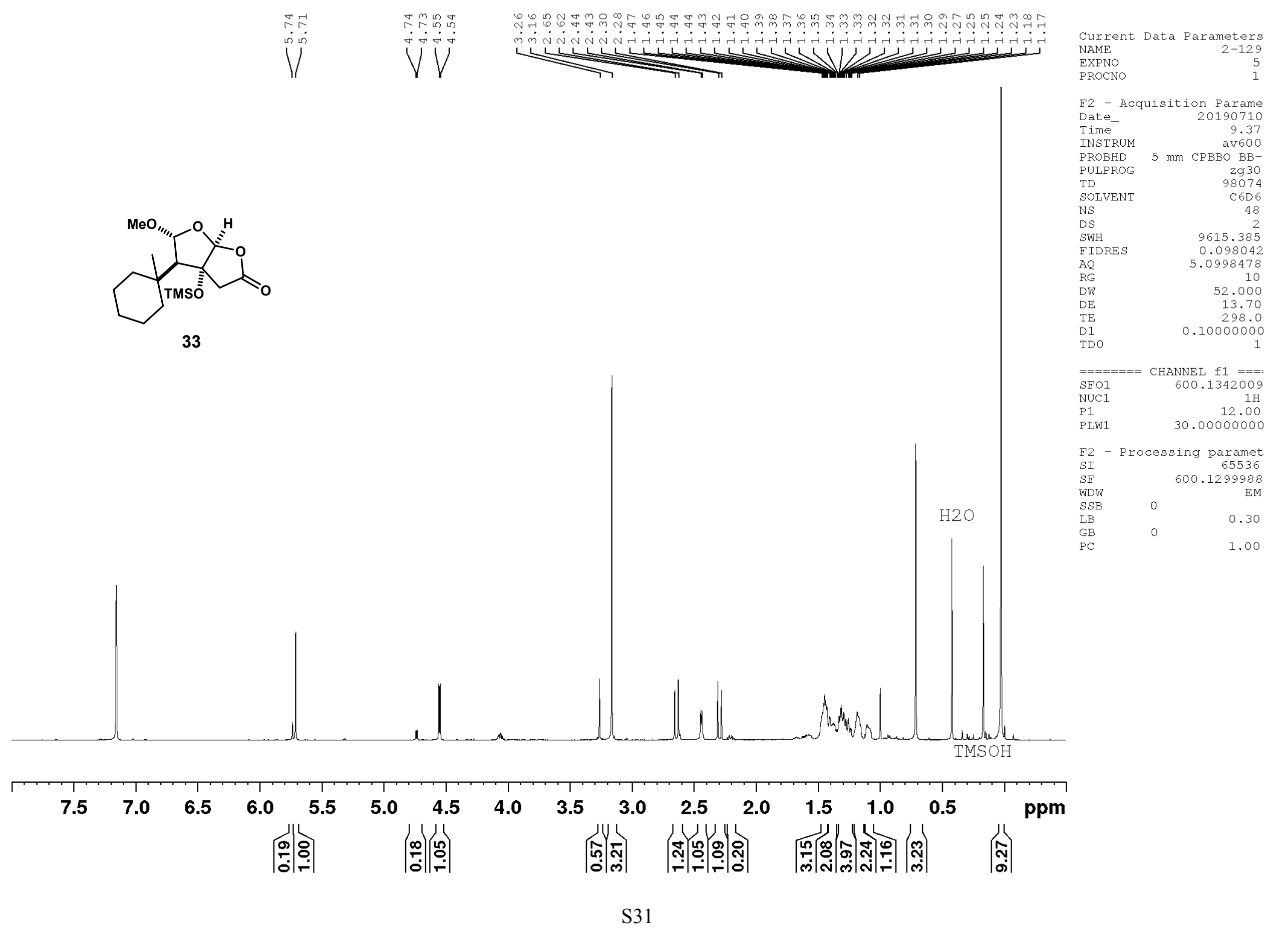




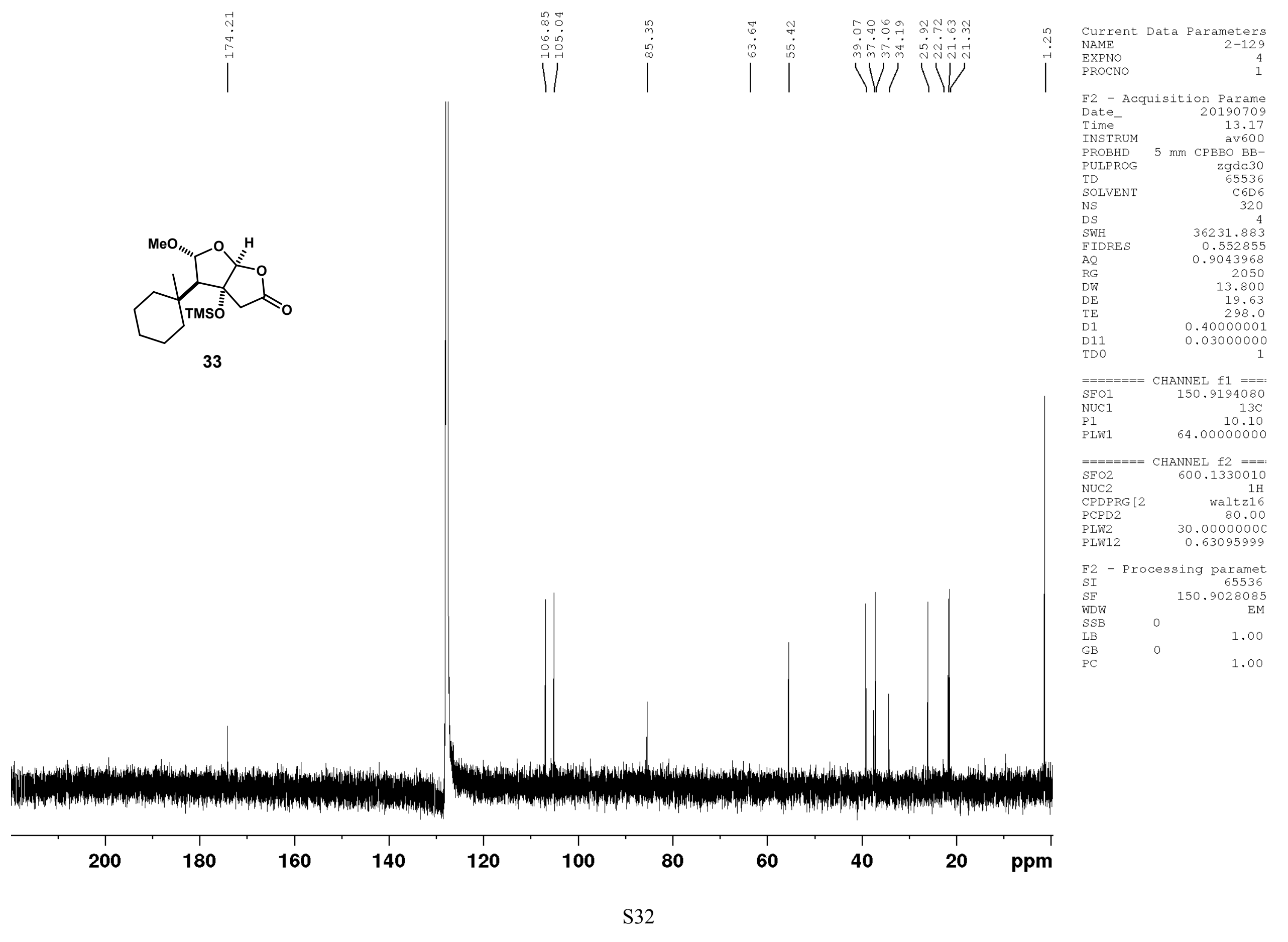



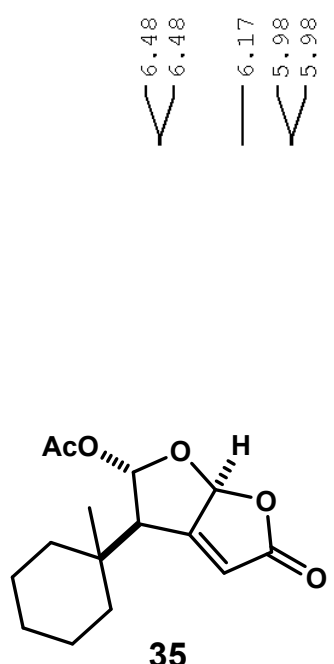

35

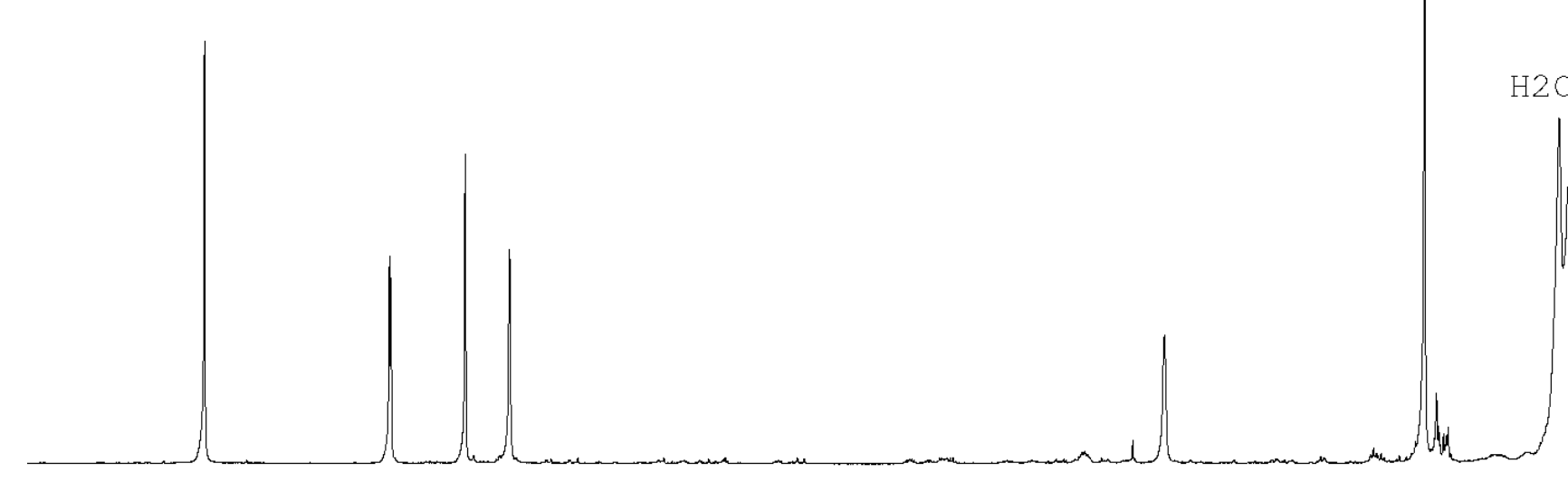

,
Current Data Parameters

E2 - Acquisition Parane Date

Time

INSTRUM

PROBHD

TD

NS

DS

2
9615.385

FIDRES

$\mathrm{AQ}$
$\mathrm{RG}$

$\mathrm{RG}$
$\mathrm{DW}$
$\mathrm{DE}$

TE
D1
TDO

$=====$

SFO1

NUC1
P1

on Parame
20190724 9.36
av 600

$\mathrm{mm} \mathrm{CPBBO} \mathrm{BB}-$

$\mathrm{zg} 30$
98074

-1
9074

32
2
.385

0.098042
5.0998478

10
52.000

13.70

0.10000000

$1 \mathrm{H}$
12.00

30.00000000

F2 - Processing paramet

SI 65536

$\begin{array}{ll}\mathrm{SF} & 600.1300364\end{array}$

EM

$\begin{array}{lll}\mathrm{SSB} & 0 & 0.30\end{array}$

PC

1.00

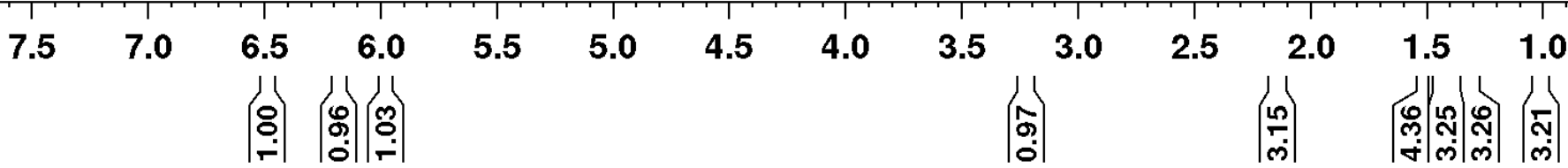



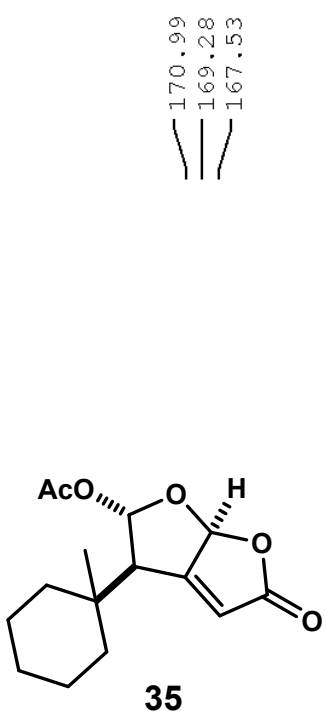

35

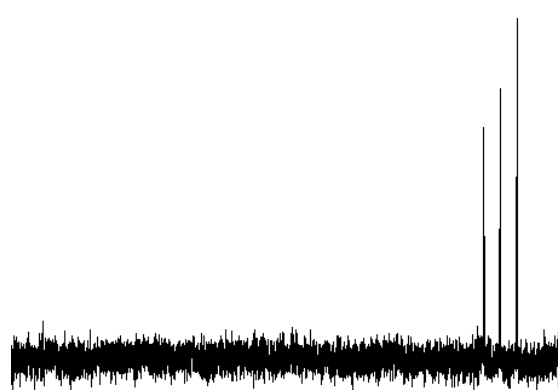

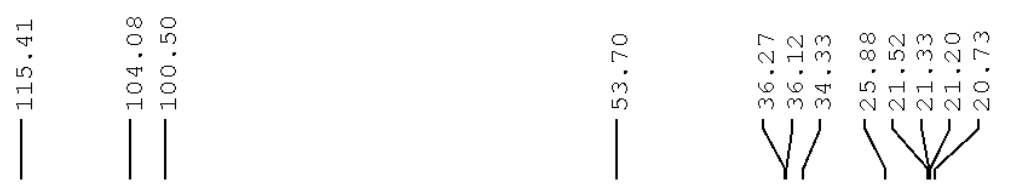

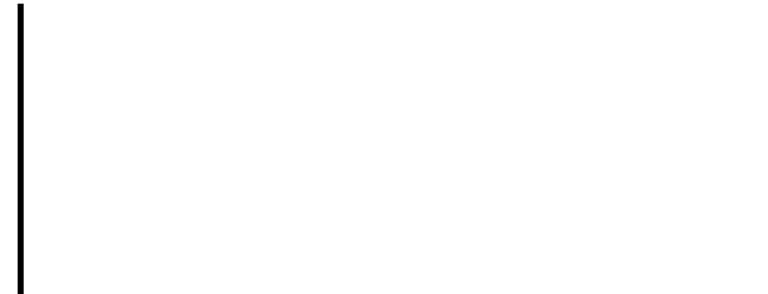

NAME

EXPN

F2 - Acquisition Parame Date_

Time

PROBHD

TD

SOLVENT

NS

DS

SWH

$\mathrm{AQ}$

RG

DW

D1
D11
TD0

$====$
SFO1

NUC1
P1

P1
PLW1

$===$

$\mathrm{SFO} 2$

CPDPRG [2

PCPD2

PLW2

PLW12 F2 - Processing paramet

SI

$S F$

WS

$\mathrm{LB}$
$\mathrm{GB}$

GB $30.0000000 C$
0.63095999

20190724
9.49
0.690

zgda 30

4713

36231.883 0.552855 2050 13.800
19.63

.03000000

$150 \cdot 9194080$

$13 \mathrm{C}$
10.10

64.00000000

CHANNEL $\mathrm{f} 2===$ 600.1330010 waltz16 150.9027941 o

1.00

1.00 

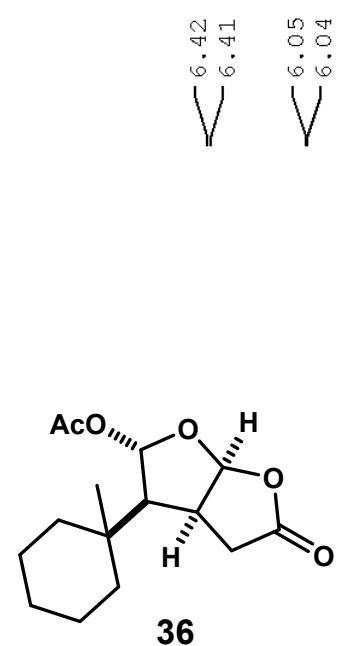

36

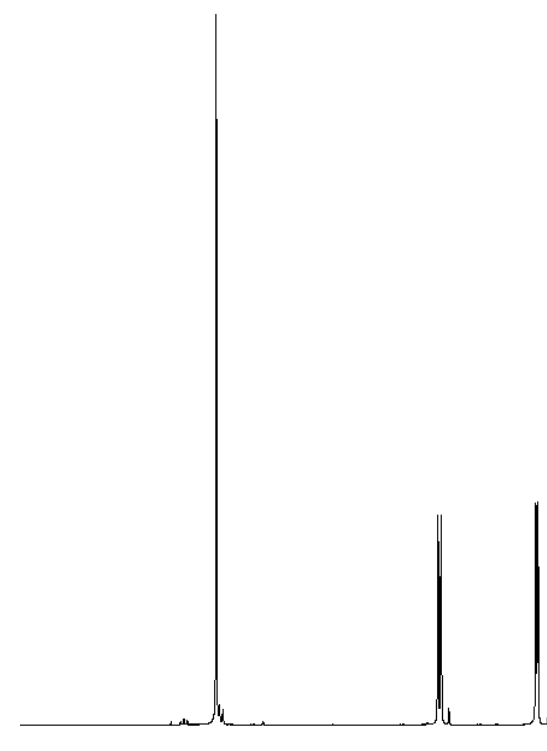

$7.5 \quad 7.0 \quad 6.5$

(용)

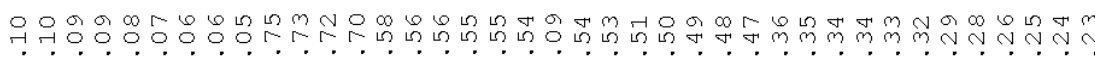
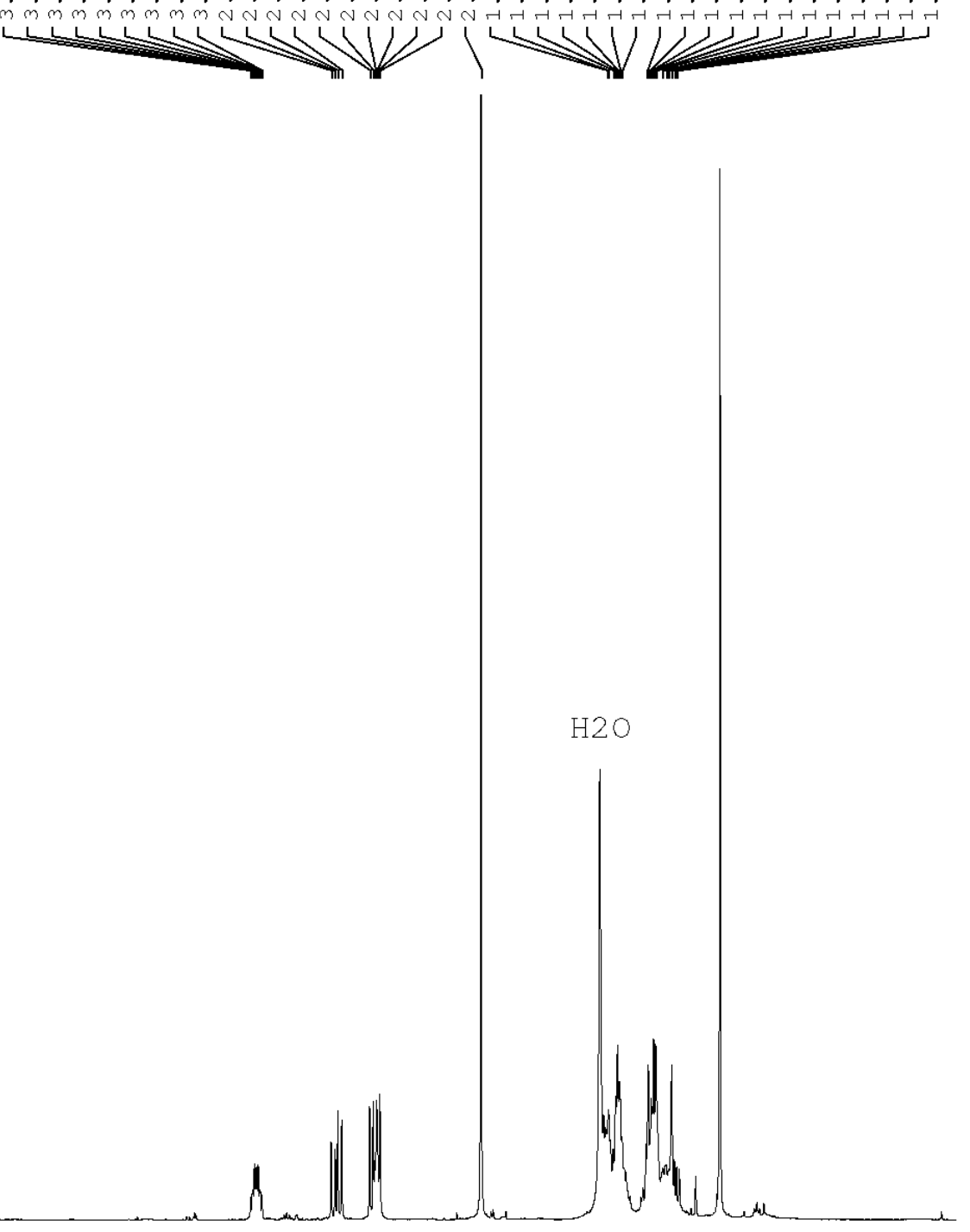

Ine

(I)

SOLVEN

S

SWH

RG

$\mathrm{DW}$

TE

D1

$===$
$\mathrm{SFO}$

NUC1

$1 \mathrm{H}$
12.00

2 - Processing paramet

SF 600.1300347

DW

0.30

1.00

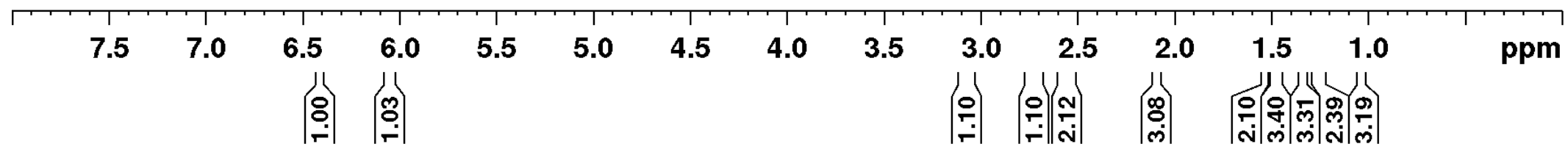




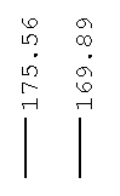

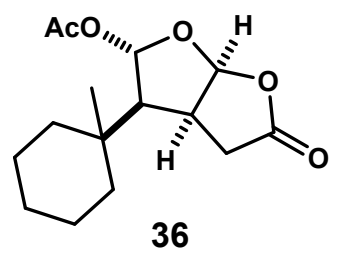

36
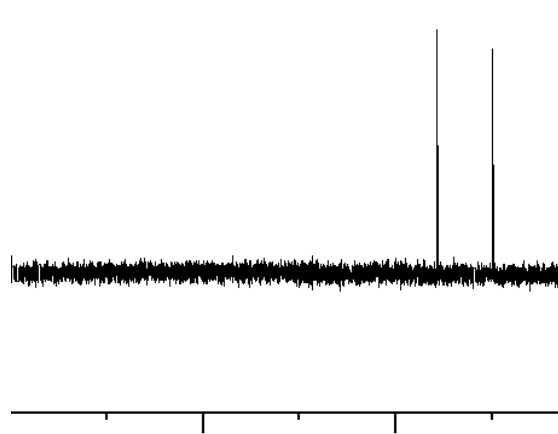

$\begin{array}{ll}\overrightarrow{7} & 0 \\ \dot{1} & 0 \\ 0 & 0 \\ \mid & 0\end{array}$

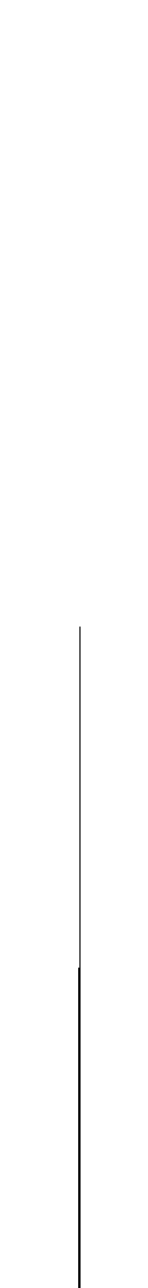

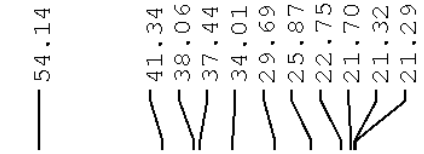
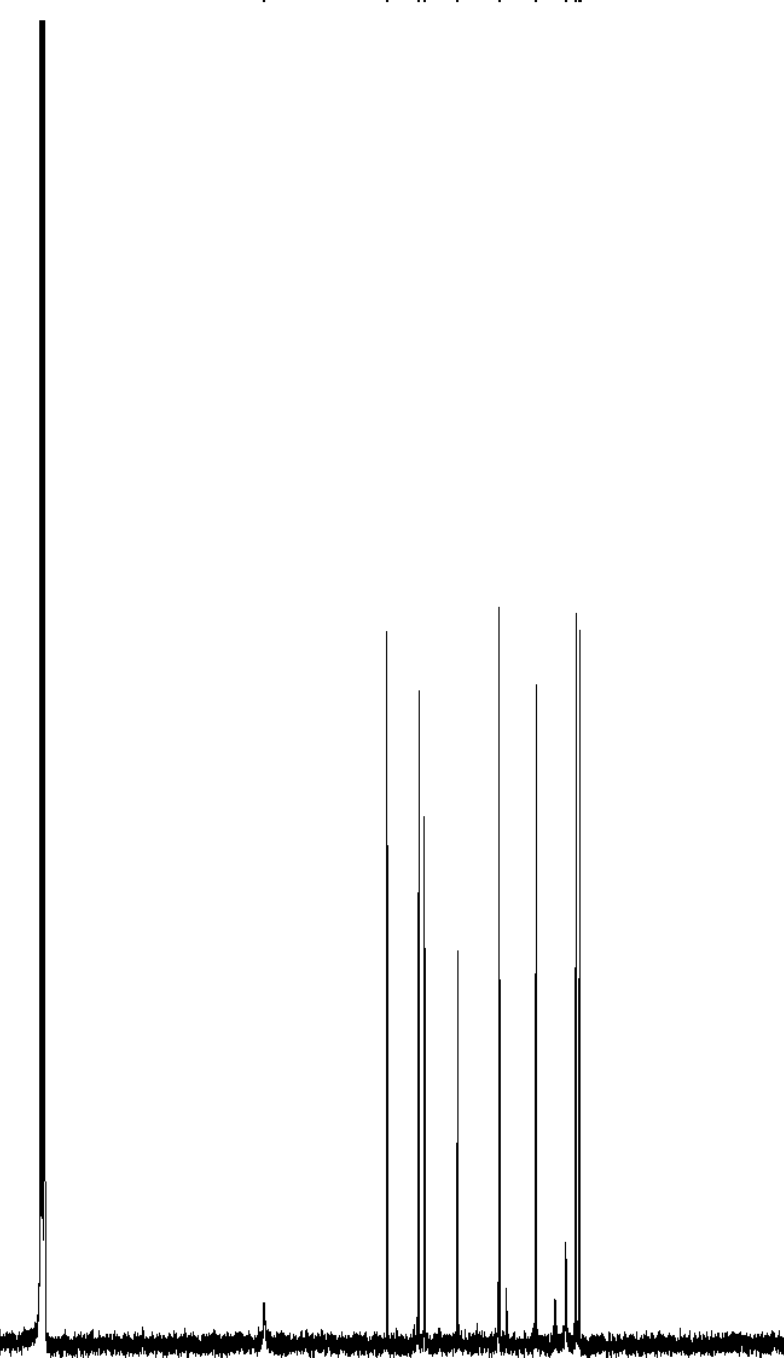

Curre

EXPNO

F2 - Acquisition Parame
Date_ 20190730 Date_

Time

INSTRUM

PROBHD

TD

TD

NS

DS

SWIDRE

AQ
RG

$\mathrm{DW}$

TE

D1

D11

$====$
SFO1

SFO1

$\mathrm{NUC1}$
$\mathrm{P} 1$

PLW1

$===$

$\mathrm{SFO} 2$

CPDPRG [2

PCPD2

PLW2

PLW2

F2

F2

SF

WDW

SS

LB

PC
Data Parameters 16.39 mm $\mathrm{CPBBO}$ BBzgdc 30 $\mathrm{CDC13}$ 36231.883 0.552855 0.9043968 2050
13.800 19.63
298.0 0.40000001 0.03000000 CHANNEL $\mathrm{f1}===$ 150.9194080 $13 \mathrm{C}$
10.10 64.00000000 CHANNEL $\mathrm{f2}===$ 600.1330010
$1 \mathrm{H}$ 80.00 0.63095999

Processing paramet 150.9027935 0

1.00

1.00 


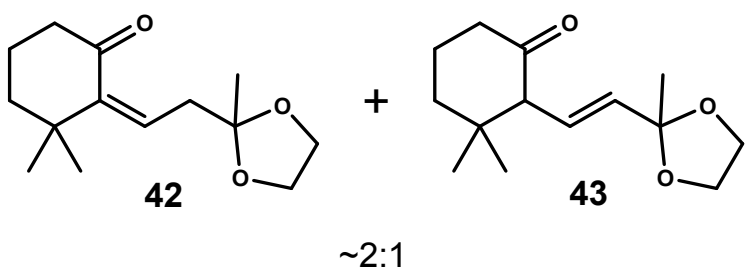

F2 - Acquisition Parame Date_ 20150717 Time $\quad 16.38$ PROBHD $5 \mathrm{~mm}$ BBO $\mathrm{BB}-1 \mathrm{H}$ PROBHD 5 $\begin{array}{lr}\text { PULPROG } & \text { Zg30 } \\ \text { TD } & 98074\end{array}$

SOLVENT

NS

$\begin{array}{lr}\text { DS } & 2 \\ \text { SWH } & 9615.385\end{array}$ 0.098042
5.0998478

F2 - Processing paranet

F2 - Processing paramet
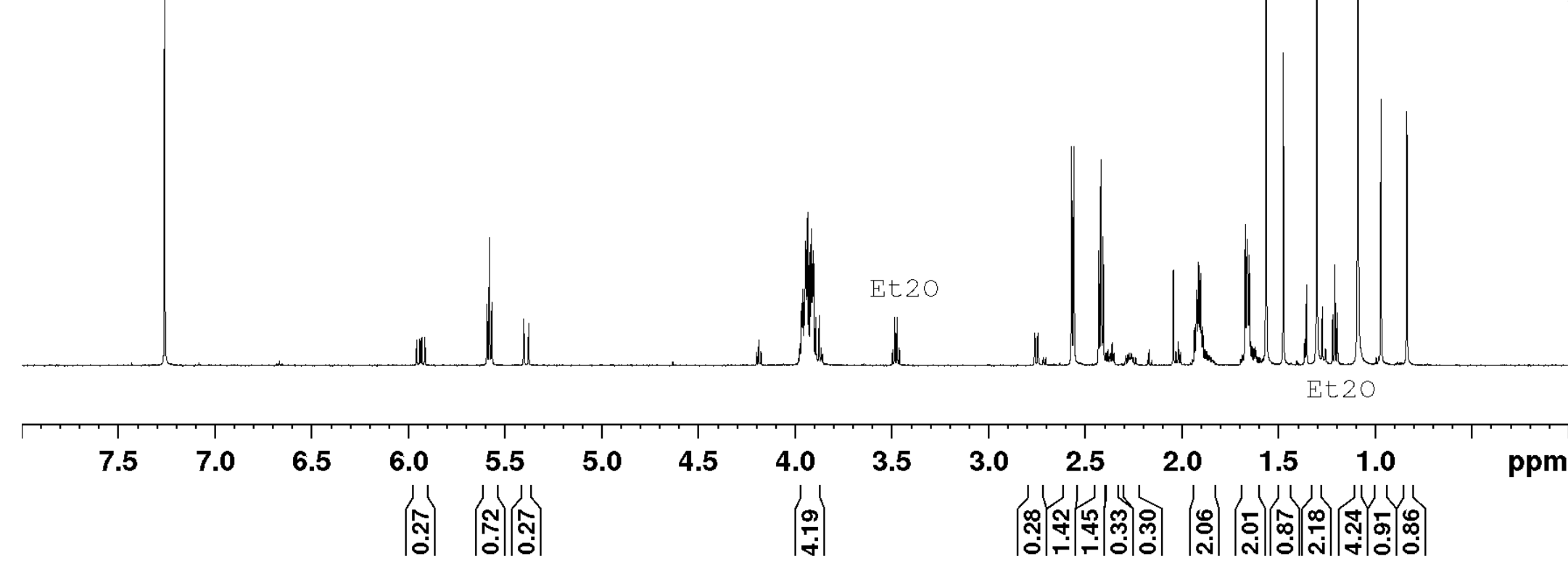
F2 - Acquisition Parame $\begin{array}{lr}\text { Time } & 11.45\end{array}$<smiles>CC1(CC[C@H]2C(=O)CCCC2(C)C)OCCO1</smiles>

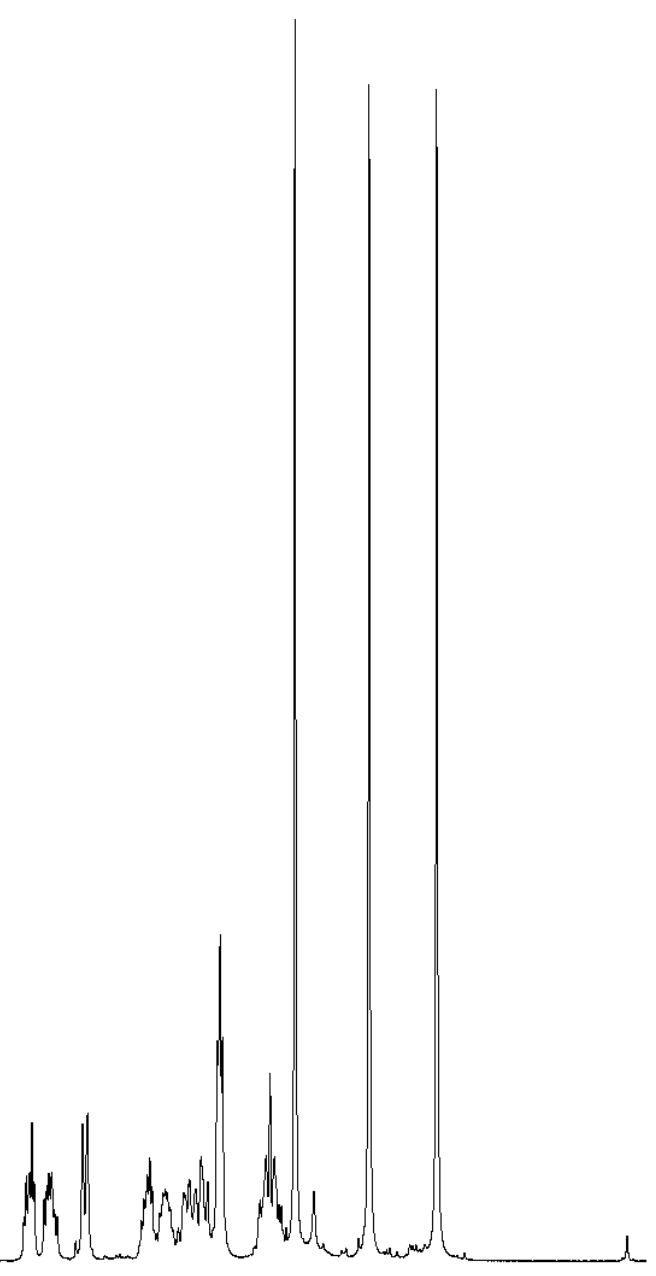

$5 \mathrm{~mm}$ TBI $1 \mathrm{H} / 13$

PULPROG $\quad$ zg30

SOLVENT

NS

SWH

F IDRE

AQ
RG

DW

DE

D1

$\mathrm{CDCl} 3$

$====$
NUC1
P1

P1
PLW1

9615.385

0.098042

5.0998478

406
52.000

6.00

0.10000000

8.00
23.01441956

F2 - Processing paramet

SI 65536

SF $\quad 600.1300291$
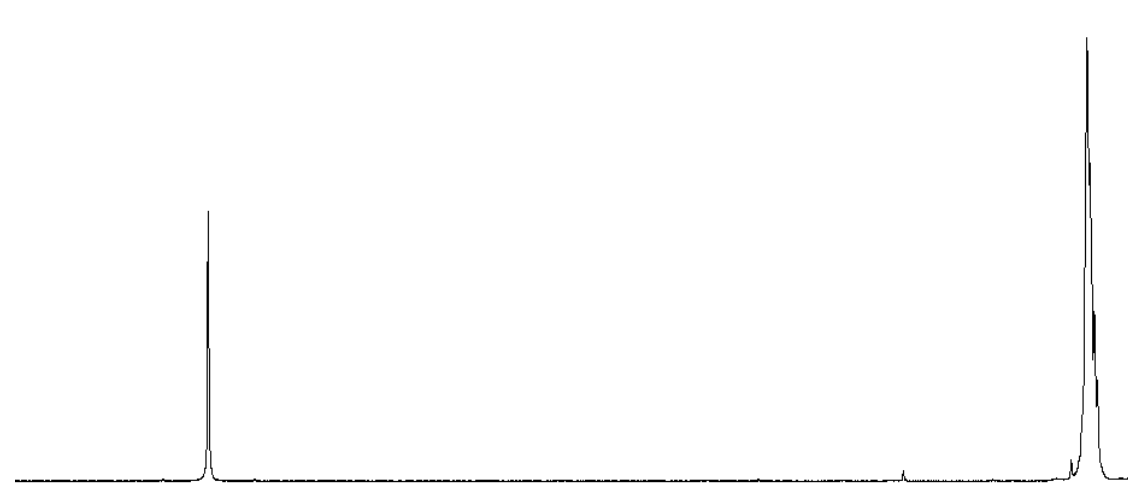

PC

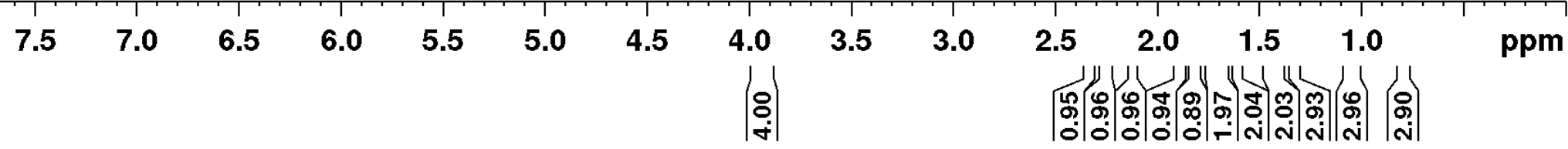




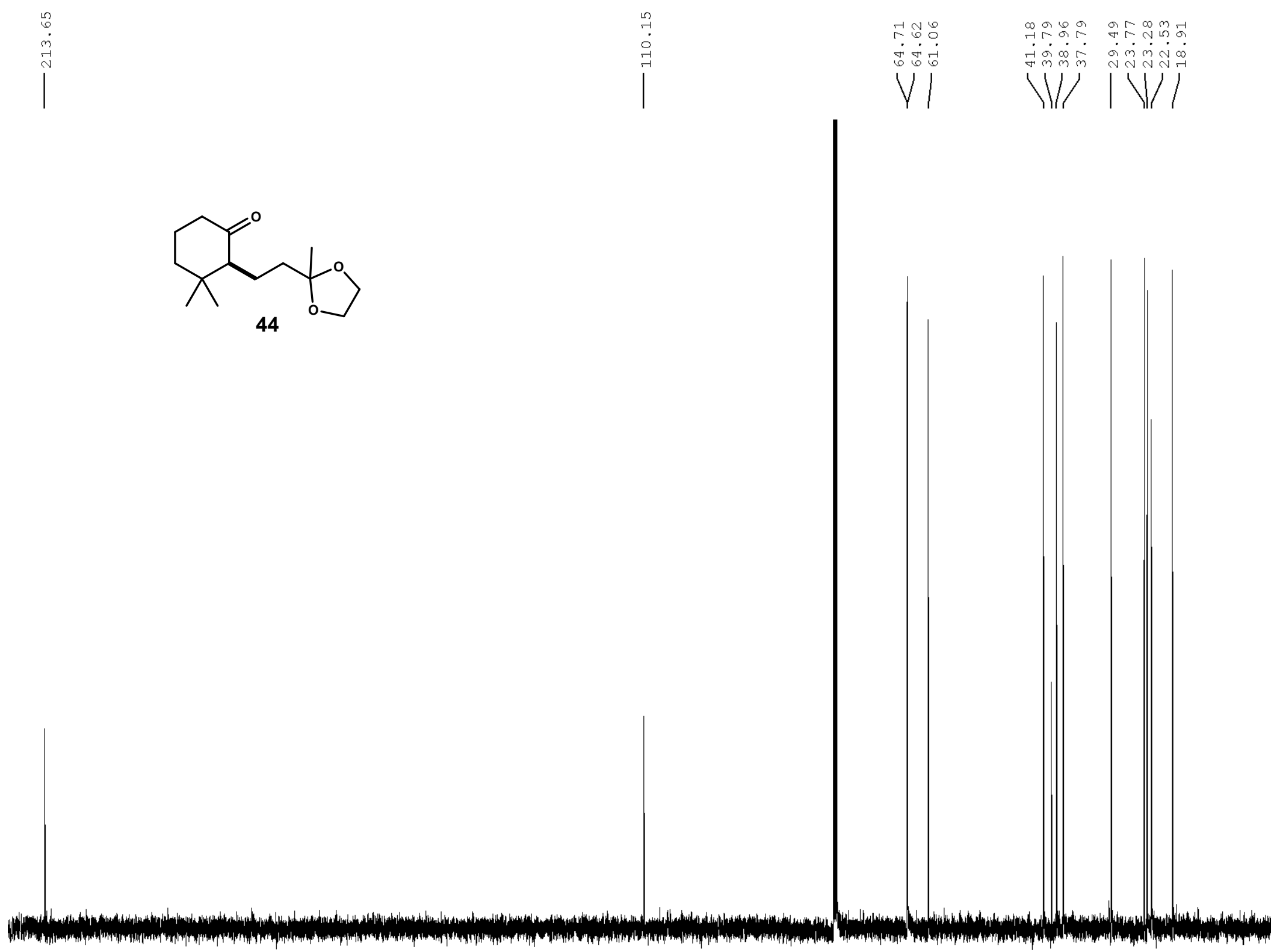

Current Data Parameters NAME APD-I-056-crud EXPNO

PROCNO

F2 - Acquisition Parame
Date_

INSTRUM cryo500

PUITROG 5 MPint $1 \mathrm{H}-$

PULPROG SpInEchopg 30 gk
TD

SOLVENT
CDCl3
DS

DS

$\begin{array}{lr}\text { DS } & 16 \\ \text { SWH } & 30303.031 \\ \text { FTDRES } & 0.462388\end{array}$

$\mathrm{AQ} \quad 1.0813440$

$\begin{array}{lr}\text { RG } & 5792.6 \\ \mathrm{DW} & 16.500\end{array}$

$\begin{array}{lr}\mathrm{DE} & 6.00 \\ \mathrm{TE} & 298.0\end{array}$

D1 $\quad 0.25000000$

d11 $\quad 0.03000000$

D16 $0.0002000 \mathrm{C}$

d17 0.00019600

MCREST 0 sec

MCWRK $\quad 0.01500000$ P2 31.00

$=====$
NUC1

$\begin{array}{lr}\text { P1 } & 15.50 \\ \text { P11 } & 500.00\end{array}$

CHANnel f1 $====$
$13 \mathrm{C}$

P12 $\quad 2000.00$

PLO $\quad 120.00$

PL1 $\quad-1.00$

SP1
S.20

SP2 2 [1] SPNAM [2] Crp60comp. 4 SPOFF1 $0 \mathrm{~Hz}$

SPOFF2 O Hz

$=======$ CHANNEL $f 2==$ Waltz16

$\mathrm{PL} 12$

ADIENT CHANNEL GPNAM [1] SINE. 100 SINE. 100

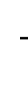

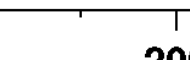




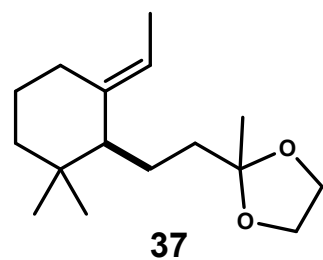

F2 - Acquisition Parane Date

Time

INSTRUM

PROBHD

PU

SOLVENT

NS

$$
\text { SWH }
$$$$
\text { FIDR }
$$

RG

DW

$\mathrm{DE}$

D1

$81-1$

F2 - Processing paramet

SI Processit

SF $\quad 600.1300283$
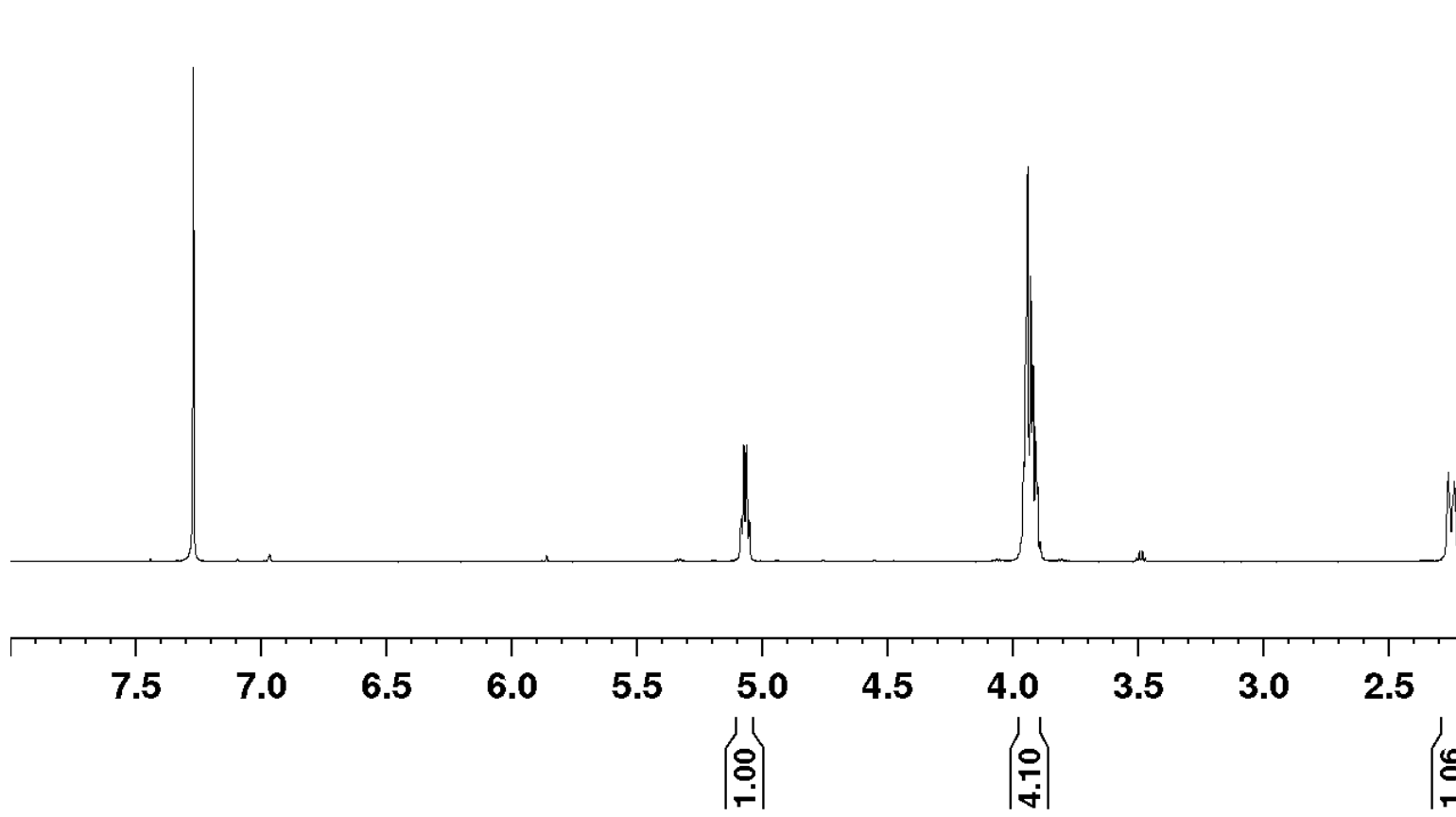

2.5
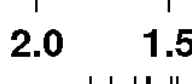

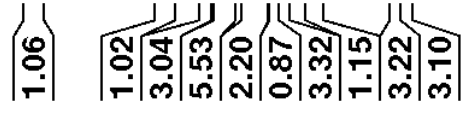

ppm

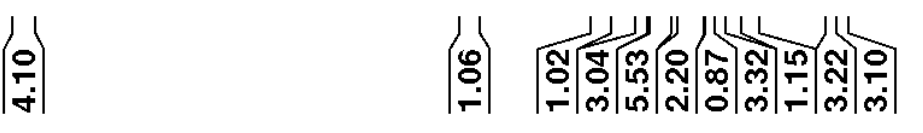




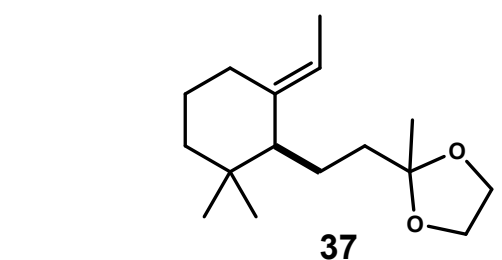

等

37
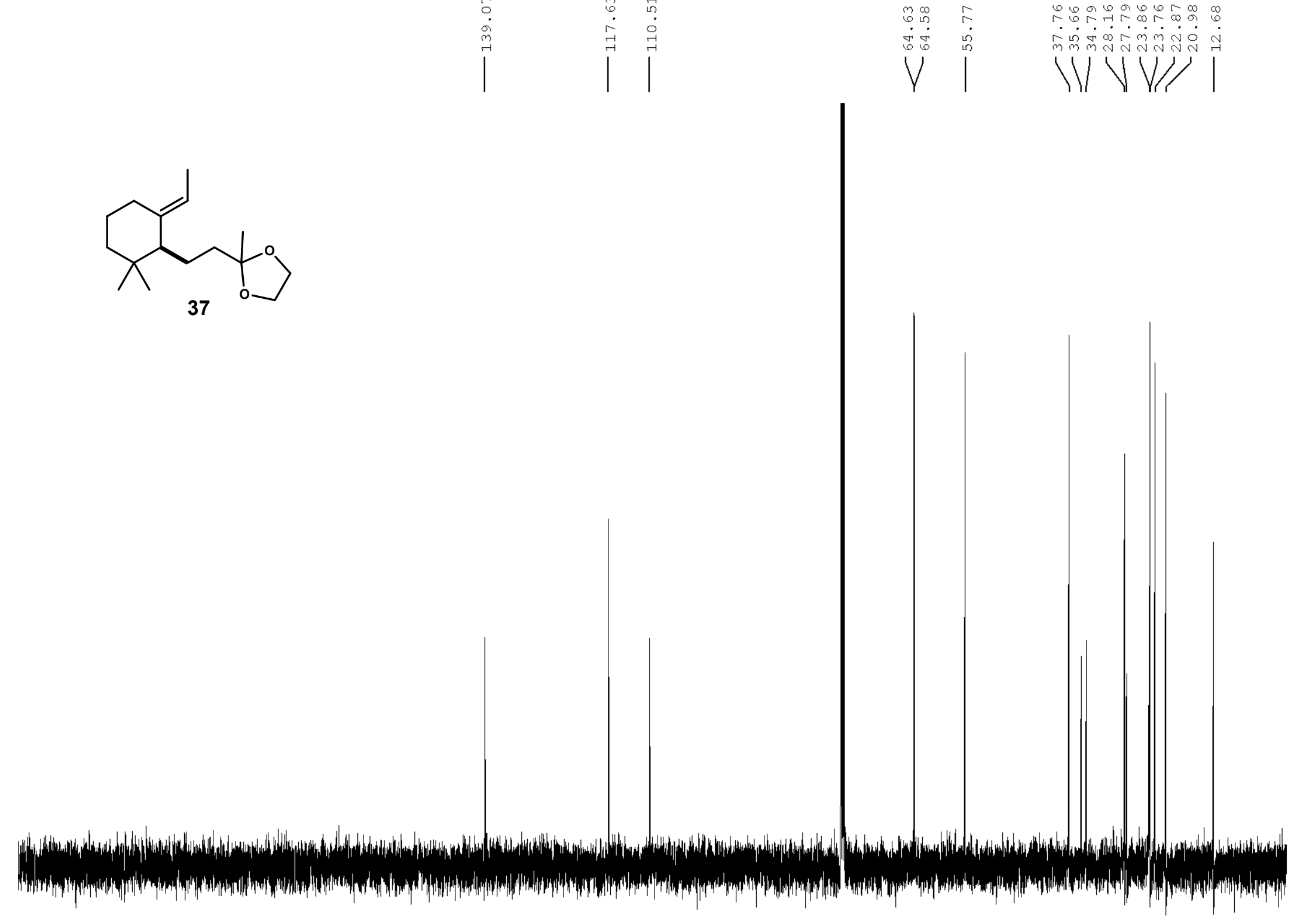

Current Data Parameters APD-I-081-1

EXPNO

PROCNO

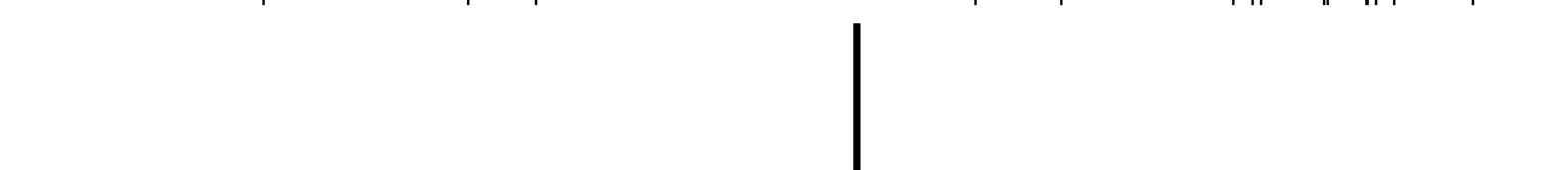
Date_ 20120820

Time PROBHD $5 \mathrm{~mm}$ CPTCI $1 \mathrm{H}-$ PULPROG SpinEchopg30gF

SOLVEN

NS

DS

FIDRES

AQ $\quad 1.0813440$

$\begin{array}{lr}\text { RG } & 4096 \\ \text { DW } & 16.500\end{array}$

$\begin{array}{lr}\mathrm{DE} & 6.00 \\ \mathrm{TE} & 298.0\end{array}$

D1 $\quad 0.25000000$

d11 $\quad 0.03000000$

D16 $\quad 0.00020000$

0.00019600

MCREST O sec

$\begin{array}{lr}\text { MCWRK } & 0.01500000 \\ \text { P2 } & 31.00\end{array}$
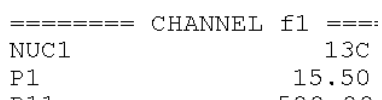

P11
P12

$\begin{array}{lr}\text { PLO } & 5000.00 \\ \text { PLI } & 120.00\end{array}$

$-1.00$

$\begin{array}{lr}\text { SF1 } & 125.7942548 \\ \text { SP1 } & 3.20\end{array}$

SPNAM [1] Crp60,0.5,20.1

SPNAM [2] CrP60 Comp. 4

SPOFF2 $0 \mathrm{~Hz}$

$=======$ CHANNEL $f 2===$

$\begin{array}{lr}\text { NUC2 } & 1 \mathrm{H} \\ \text { PCPD2 } & 100.00\end{array}$

$\mathrm{PL} 12$

$\mathrm{SEO} 2$

500.2225011

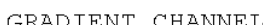

GPNAM [1] GPNAM[2] SINE. 100 GPX1 


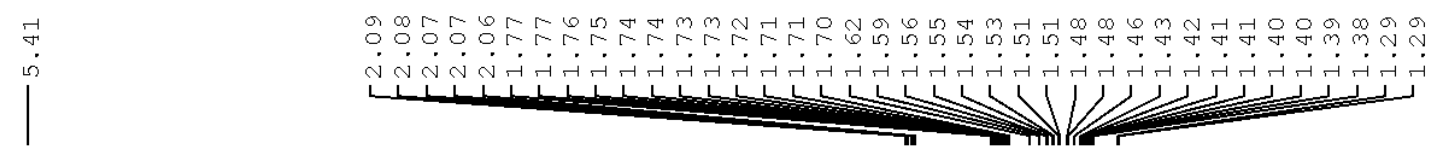

$\begin{array}{lr}\text { Current } & \text { Data Parameters } \\ \text { NAME } & \text { APD-I-107-1 } \\ \text { EXPNO } & 1 \\ \text { PROCNO } & 1\end{array}$
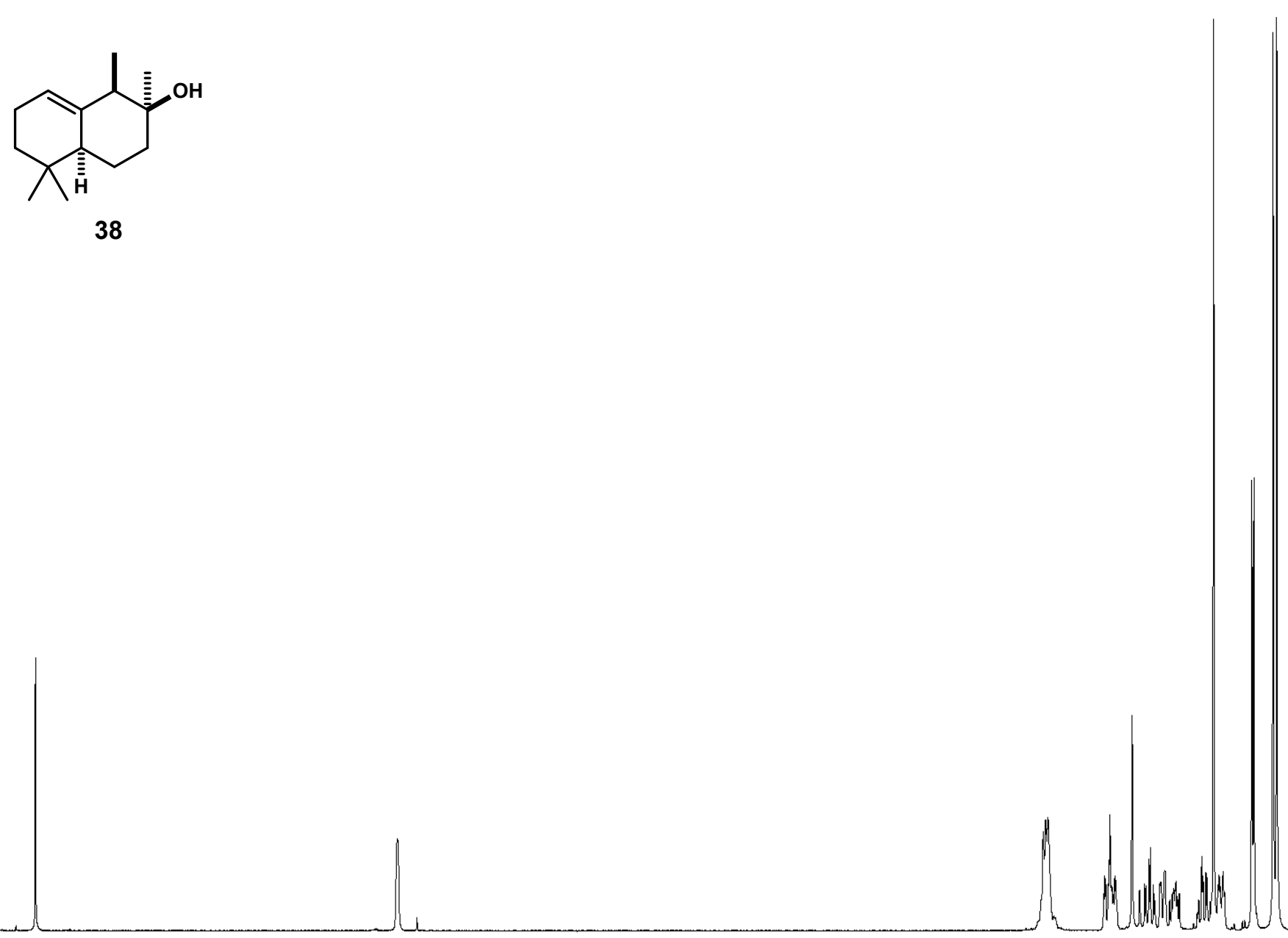

F2 - Acquisition Parame

Date

20120906
16.15

IIMSTRUM

PROBHD

PROBHD

PUT

TD
SOLVENT

NS

$\begin{array}{lr}\text { DS } & 2 \\ \text { SWH } & 9615.385 \\ \text { FIDRES } & 0.098042\end{array}$

$\mathrm{AQ} \quad 5.0998478$

38

RW

$\mathrm{DE}$

D1

52.000

6.00

0.10000000

$=======$ CHANNEL $\mathrm{f} 1====$
NUC1

PLW

23.01441956

F2 - Processing paramet

SI

SF $\quad 600.1300283$

WDW EM

SSB

L

$\mathrm{PC}$

0.30

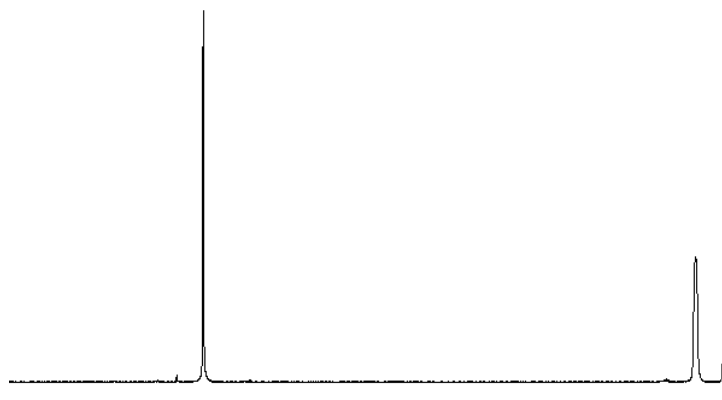

1.00

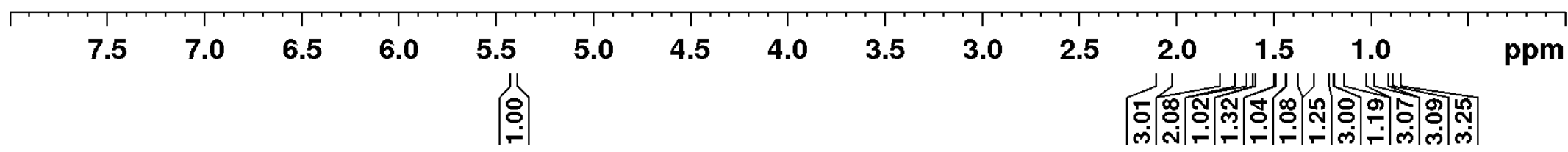


38
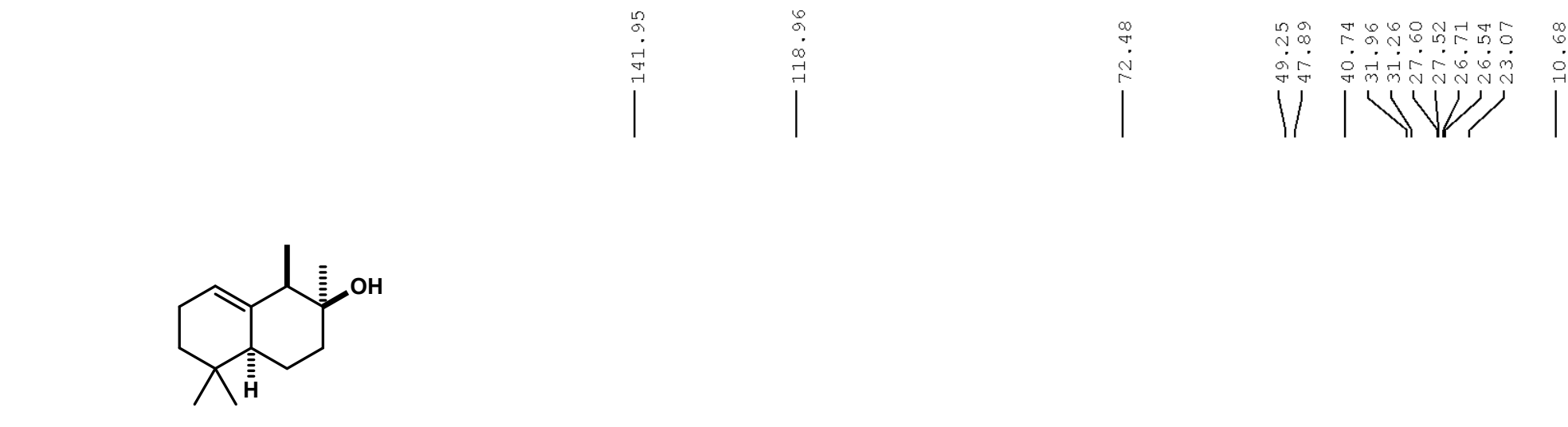

rent Data Parameters NAME EXPNO

F2 - Acquisition Parame Date

INSTRUM

PROBHD 65536 SOLVENT

NS

SWH

FIDRES

$\mathrm{AQ}$

RG

$\mathrm{DW}$
$\mathrm{DE}$

$\mathrm{DE}$

D1

d11

d17

MCREST

P2

$=====$
NuC1

P1

P11
P12
PLO

PL1

SFO1

SP 1

Crp60, 0.5,20.1

SPNAM[2] Crp60comp.4

SPOFF 1

$\begin{array}{ll}0 & \mathrm{~Hz} \\ 0 & \mathrm{~Hz}\end{array}$

f2 $===$ CPDPRG [2 waltz16 NUC2

PCP 2

$\mathrm{PL2}$

$\mathrm{PL} 12$
$\mathrm{SFO} 2$

30303.031

0.462388
1.0813440 4597.6

16.500
6.00

298.0

.03000000 .00020000

0.01500000 31.00 f1 $===$ 15.50
500.00 2000.00 -1.00
125.7942548 24.60
500.2225011

$====$ GRADIENT CHANNET GPNAM [1] GPNAM [2] GPX1 GPX2 GPY1 GP Z1

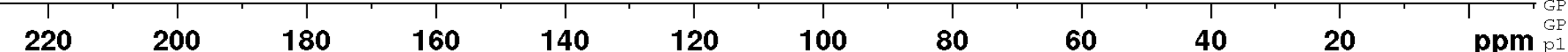

ppm 15 


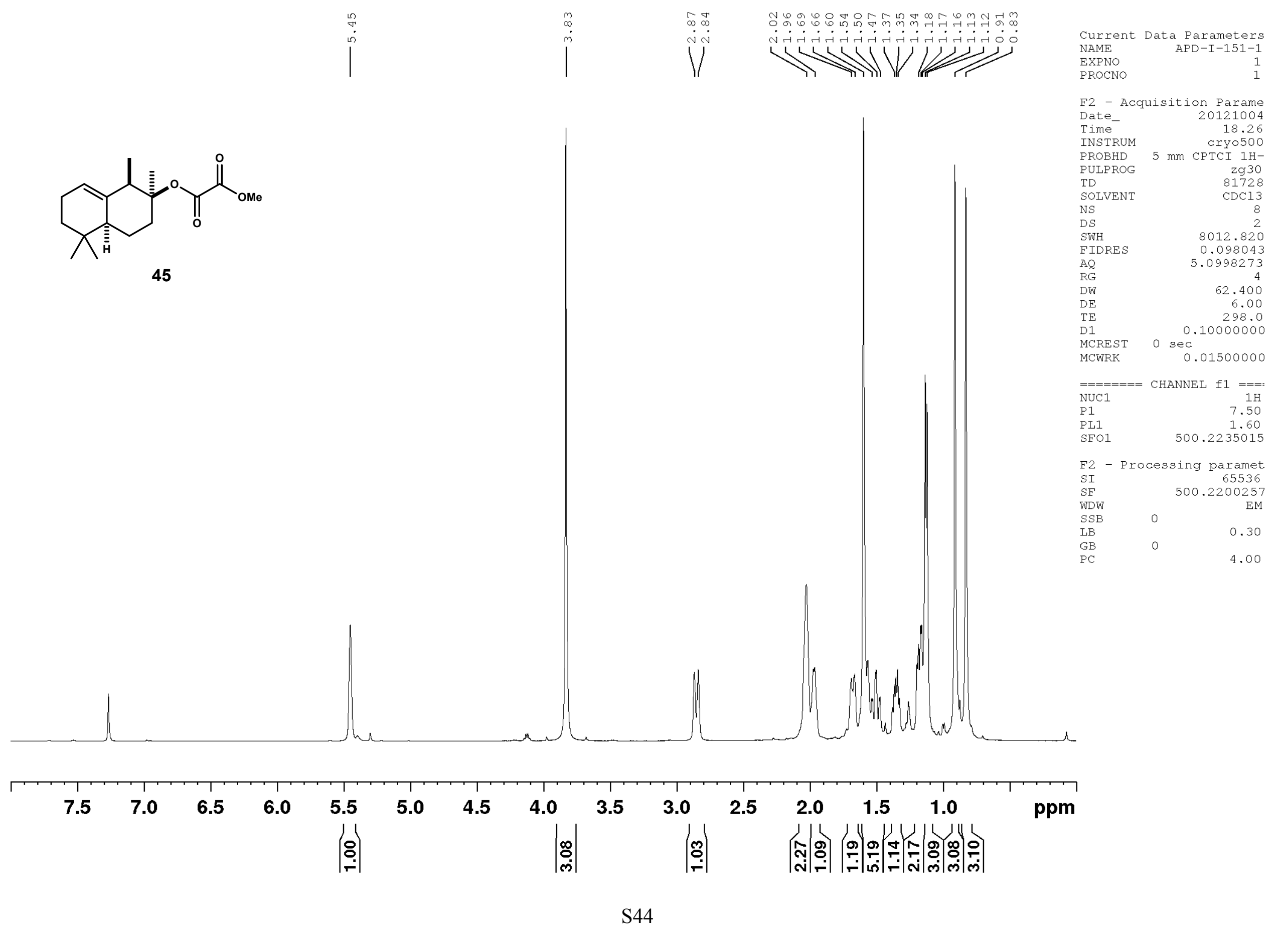




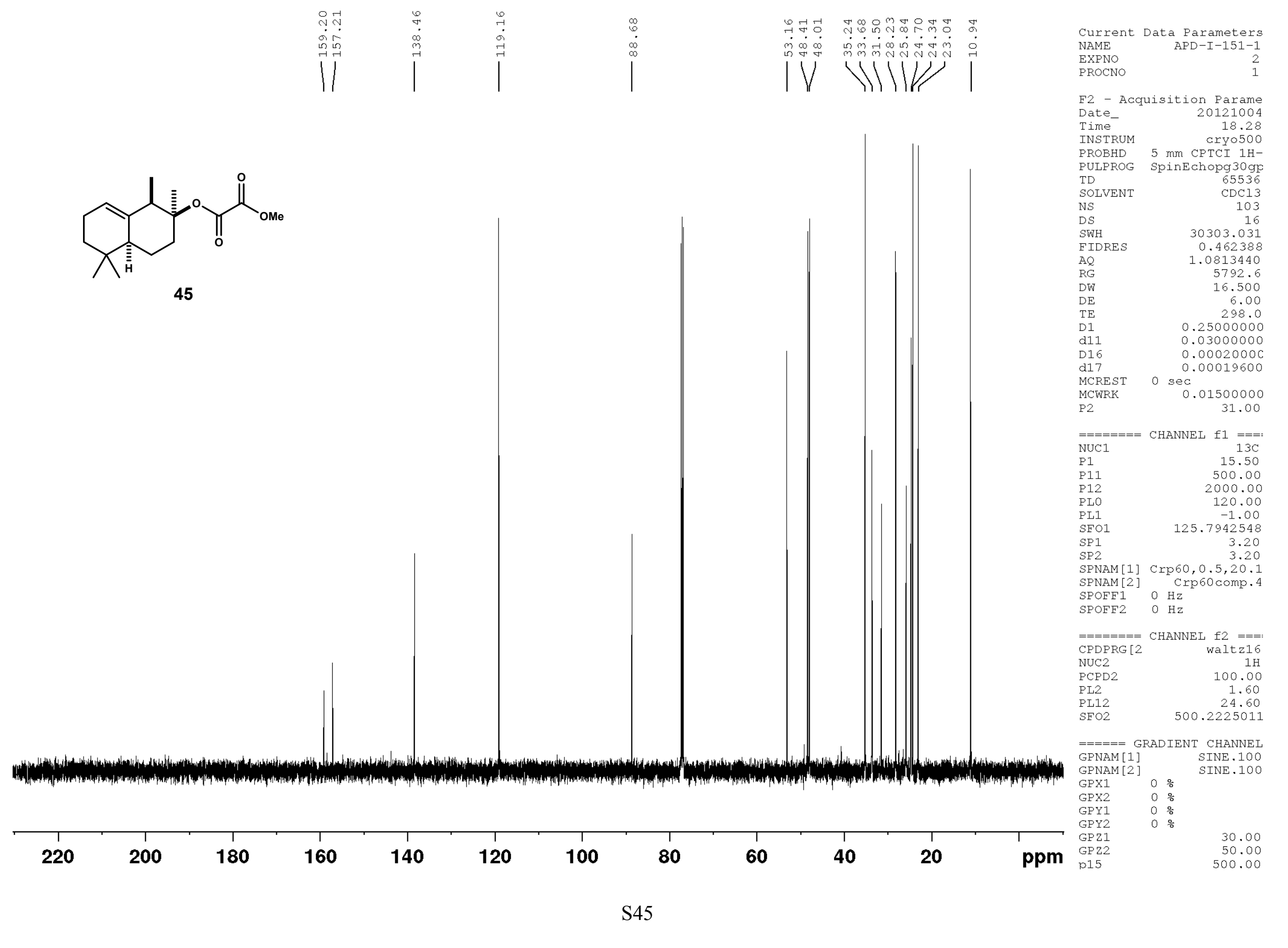




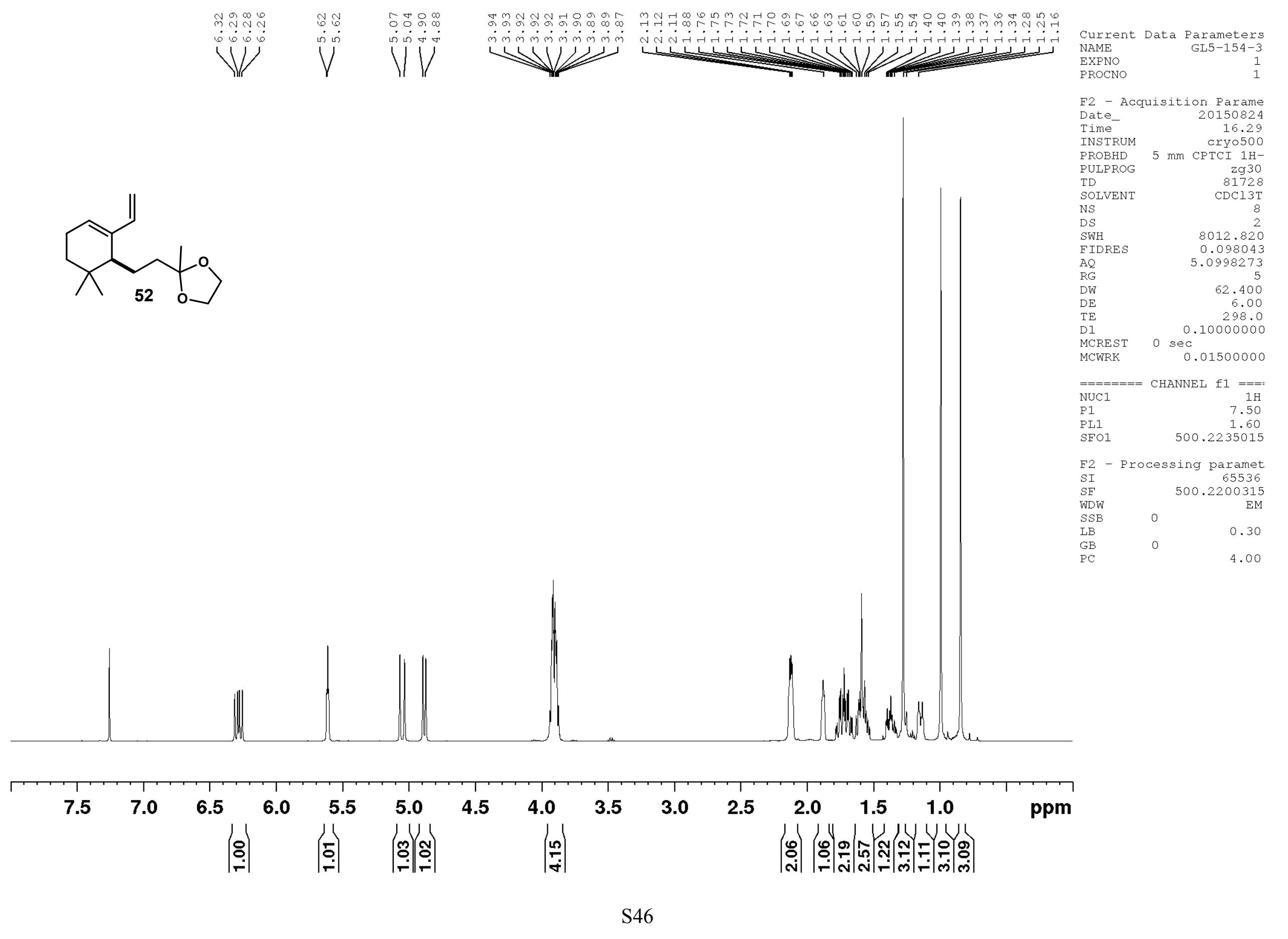




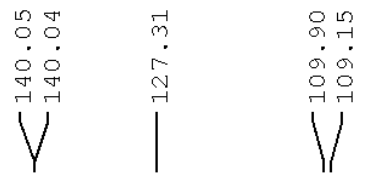
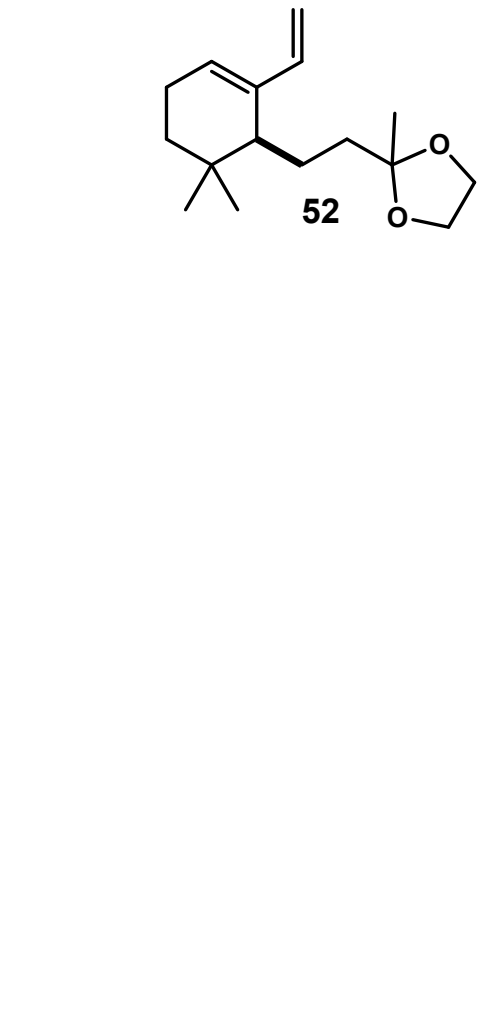

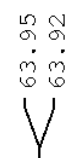

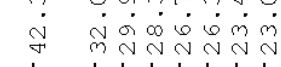

livk

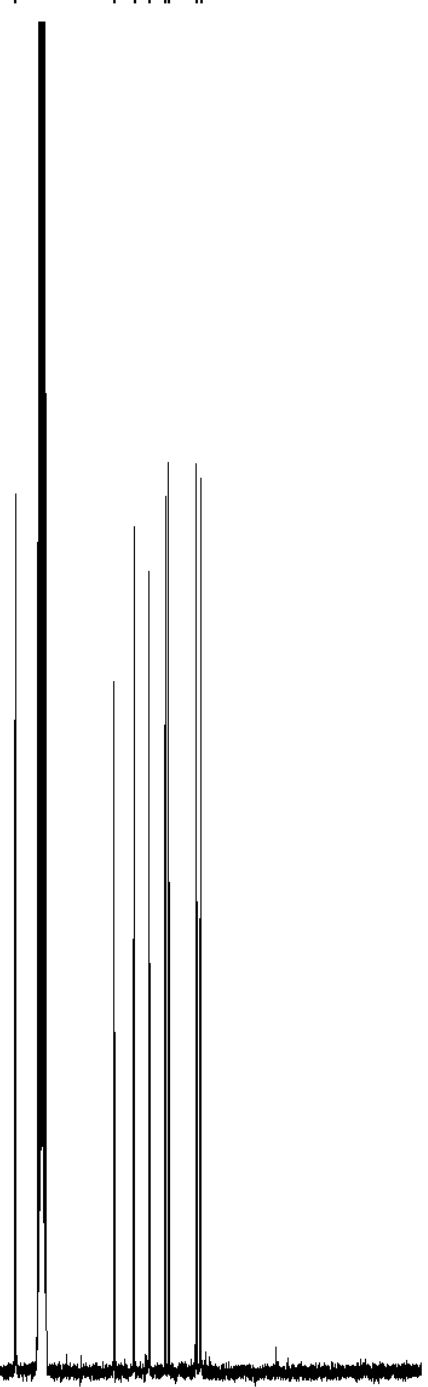

Current Data Par

NAME

EXPNO

F2 - Acquisition Parame Date_

Time

TRSTRM

PROBHD

TD
SOLVENT

NS

DS

FIDRE

AQ
RG
DW

$\mathrm{DE}$

D1

TD11

$====$
SFO1

SFO1

P1

P1 1

$===$

$\mathrm{SFO} 2$

CPDPRG [

PCPD 2

PLT212

F2 - Processing paramet

SF

WDW

SSB

$\mathrm{LB}$
$\mathrm{GB}$

PC
GL5-162-3dmso 19.31
2.600 mm $\mathrm{CPBBO} \mathrm{BB}-$ 20 zgdc 30 DMSO 36231.883 0.552855 0.9043968 2050
13.800 19.65
298.0 0.400000 0.03000000 1 150.9194075 $13 \mathrm{C}$
10.00 64.00000000 CHANNEL $\mathrm{f} 2===$ 600.1330010 $1 \mathrm{H}$
waltz16 $20.0000000 \mathrm{C}$ 36000001 65536 150.9028809 0 1.00 1.00 


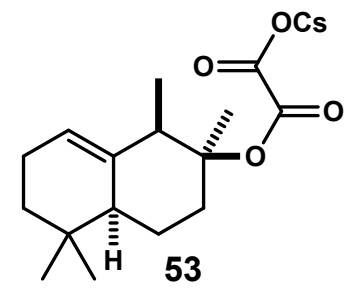

F2 - Acquisition Parame

Date__ 20160428$$
\begin{aligned}
& \text { Time } \\
& \text { INSTRUM }
\end{aligned}
$$

PROBHD

-39
70

$\begin{array}{lr}\text { PG } & \text { Z } 30 \\ \text { TD } & 98074\end{array}$

SOLVENT

NS

SWH

FIDRE
$\mathrm{AQ}$

AQ
RG
$D W$
DF

$\mathrm{DE}$
$\mathrm{TE}$

TE

TDO

$====$
SFO1

$\mathrm{P} 1$

DMSO

PLWT1

9615.385

0.098042

5.0998478

52.000

13.70

$0.1000000 \mathrm{C}$

20.00000000

F2 - Processing paramet

$\begin{array}{lr}\text { SI } & 65536 \\ \text { SF } & 600.1300295\end{array}$

WDW EM

PC

0.30

1.00

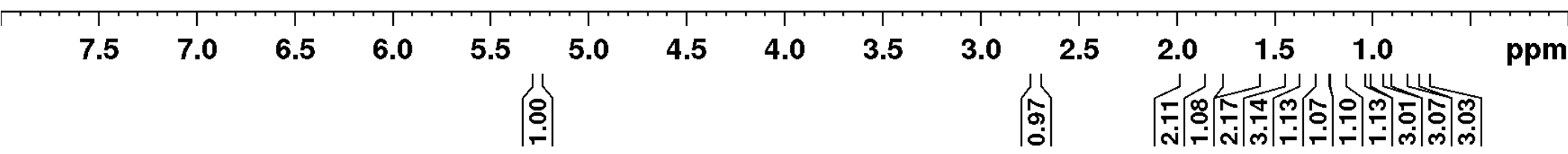




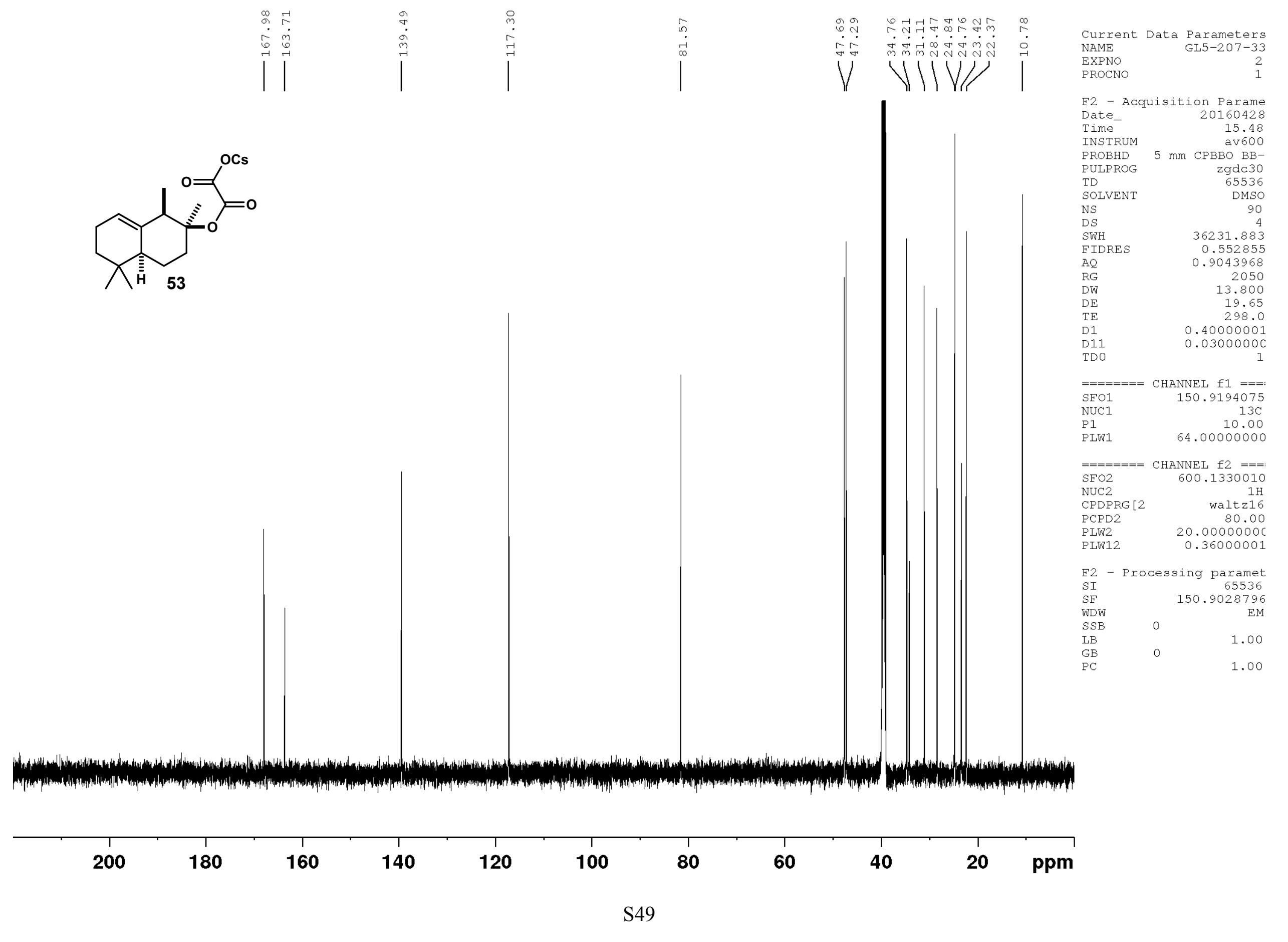




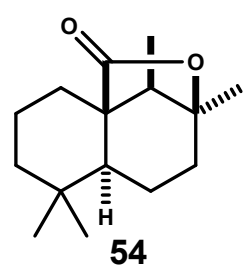
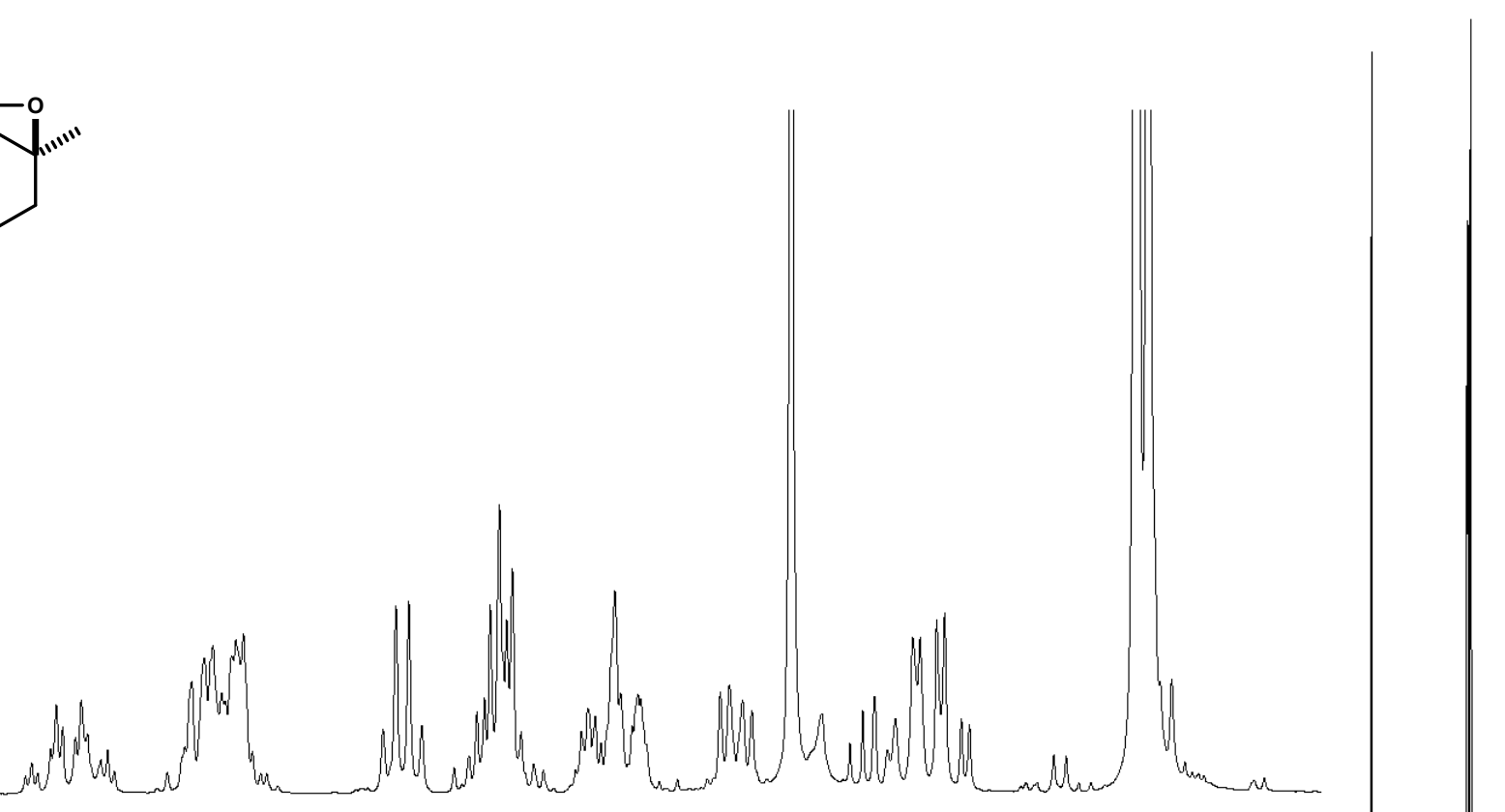

F2 -

Date

INSTRUM

PROBHD

PUIP

TD
SOLVENT

NS

DS

FIDRES

$\mathrm{AQ}$
$\mathrm{RG}$

RW

$\mathrm{DE}$

D1

MCRE

MCWR

on Parame 15.13
aryo 500

N

NUC1

$\mathrm{P} 1$
$\mathrm{PL} 1$

PL1
SFO1

$\mathrm{mm}$ CPTCI $1 \mathrm{H}-$

$\mathrm{zg} 30$
81728

SI

SF $\quad 500.2200313$

WD

$\mathrm{LB}$

GB

$$
0
$$

0.30

4.00

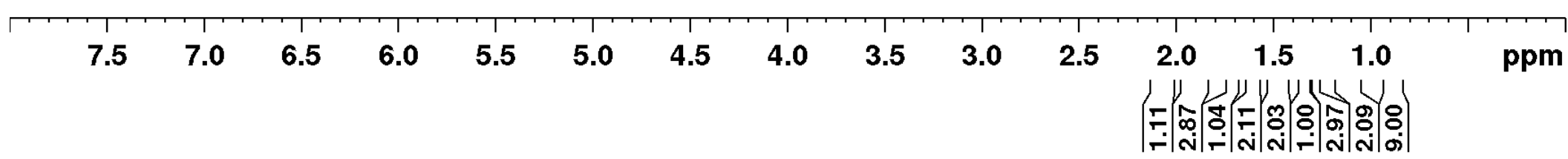




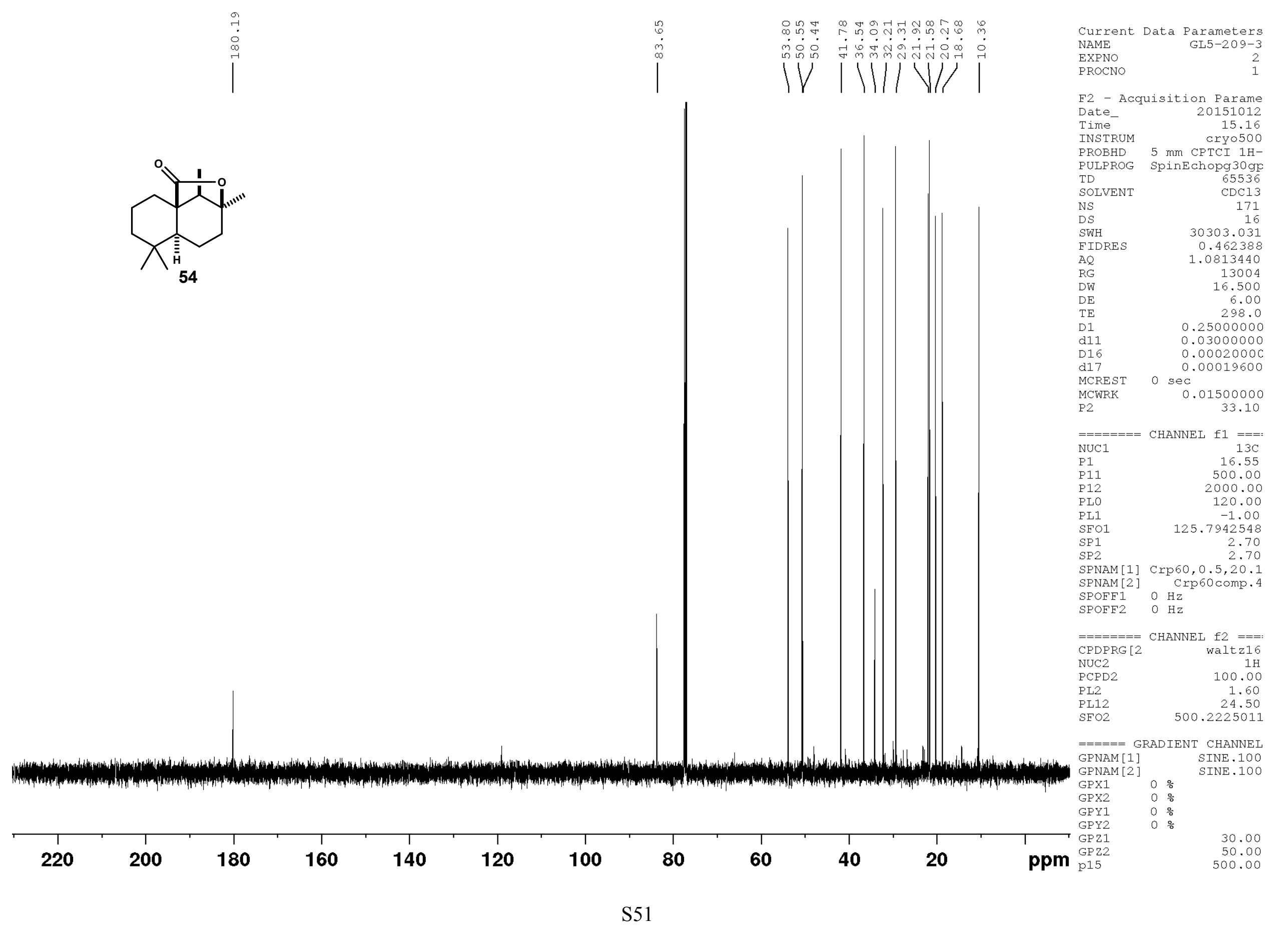




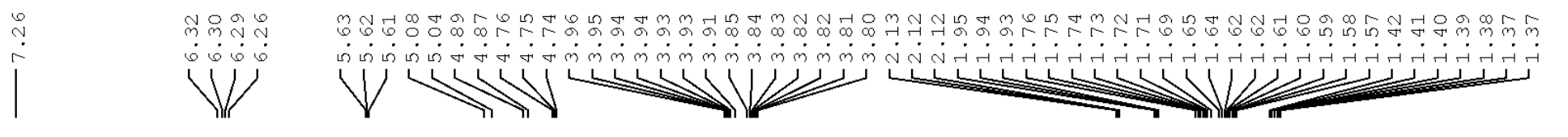

$\begin{array}{lr}\text { Current } & \text { Data } \\ \text { NAME } & \text { GL5-217- } \\ \text { EXPNO } & 1 \\ \text { PROCNO } & 1\end{array}$

F2 - Acquisition Parame

Date__ 20151019

11.32
TinsTRUM

cryo500

CPTCI $1 \mathrm{H}-$

TD

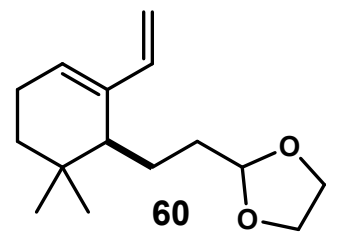

NS

DS

SWH

AQ

RG

DW

$\mathrm{DE}$

D1 0.10000000

MCREST 0 sec

0.01500000

NUC1

P1
PL1
SFO1

$===$
$1 \mathrm{H}$
.50
.60

F2 - Processing paramet

SI 65536

$\begin{array}{ll}\text { SF } & 500.2200312\end{array}$
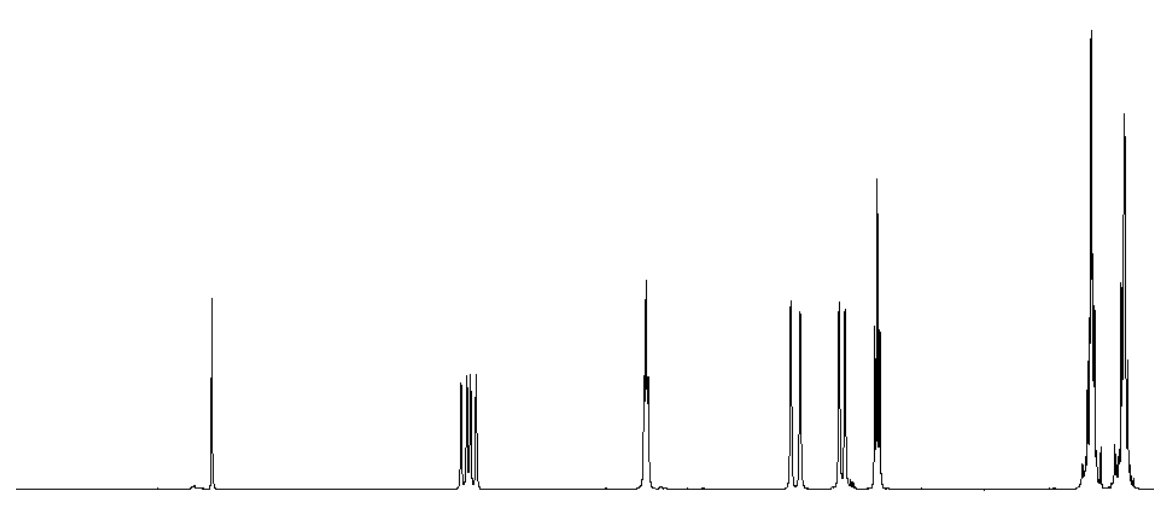

0.30

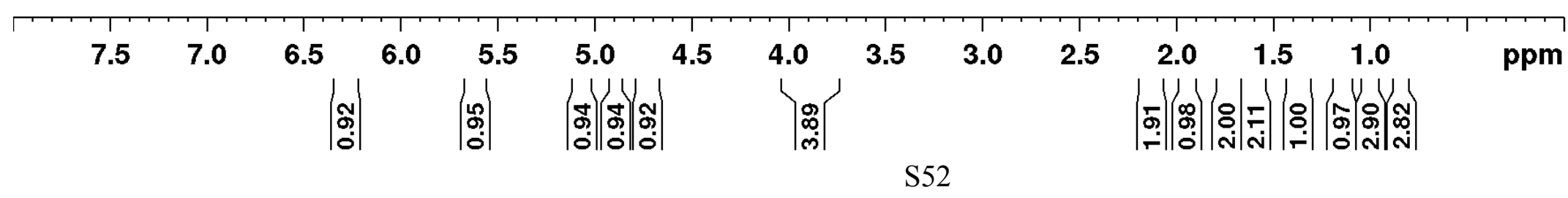



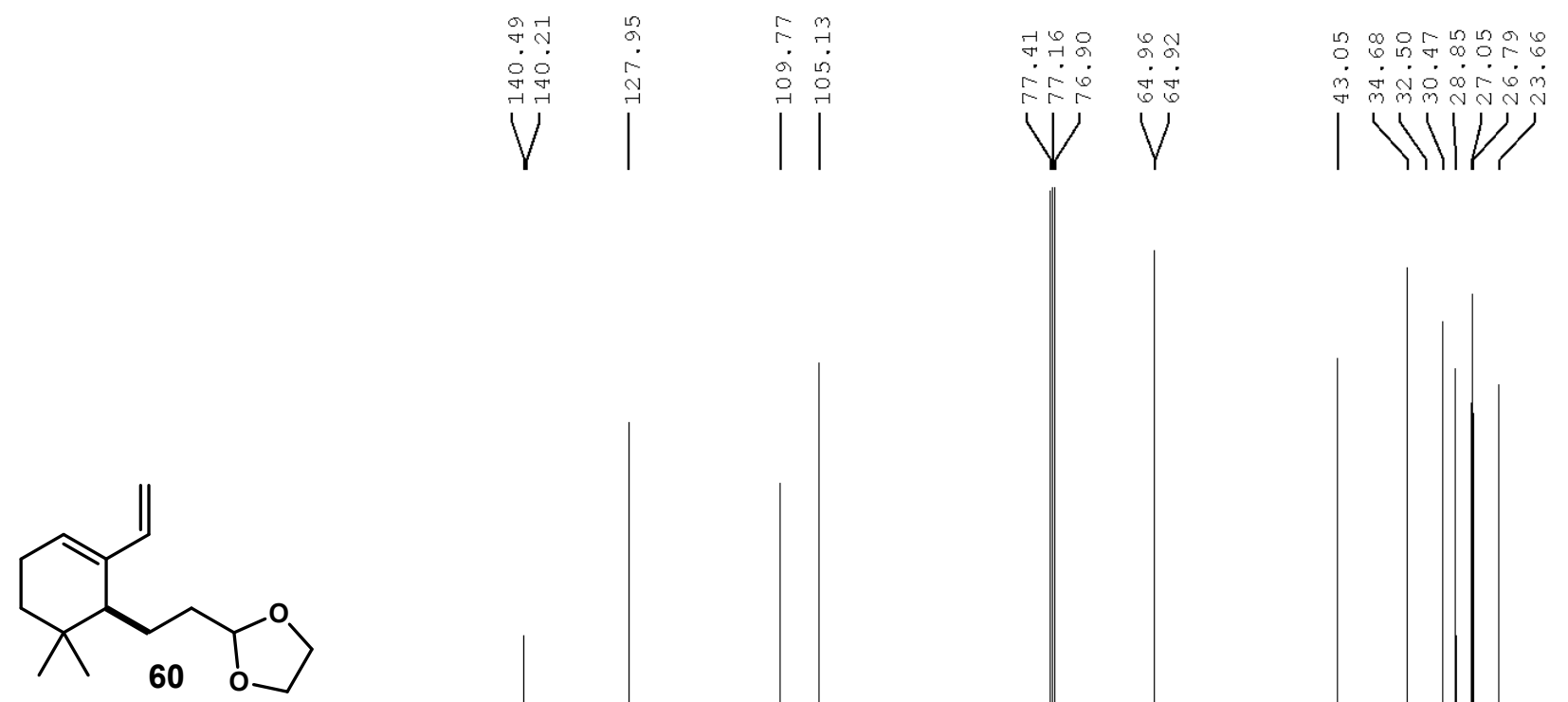

NAME

EXPNO

PROCNO

F2 - Acquisition Parame

Time-

INSTRUM PUTPROG 5 min CPTCI $1 \mathrm{H}-$

SOLVENT

NS

$\begin{array}{lr}\text { DS } & 16 \\ \text { SWH } & 30303.031\end{array}$

30303.031
0.462388

AQ

DW

$\mathrm{DE}$

D1

d11

d17

MCREST

MCWR

P2

1.0813440

10321.3

16.500

298.0

0.25000000

0.03000000

$0.0002000 \mathrm{C}$

0.00019600

$=====$
NUC1

P1

P11
P12
PLO

PL1

SP1

SPNAM [1] Crp60, 0.5,20.1 SPNAM [2] Crp60comp.4

$\begin{array}{ll}\text { SPOFF1 } & 0 \mathrm{~Hz} \\ \text { SPOFF2 } & 0 \mathrm{~Hz}\end{array}$

$=======$ CHANNEL $\mathrm{f} 2 \quad===:$
CPDPRG 2 waltzl6 $\begin{array}{lr}\text { NUC2 } & 1 \mathrm{H} \\ & 100.00\end{array}$ $\begin{array}{lr}\text { PCPD2 } & 100.00 \\ & 1.60\end{array}$

PL2

$\mathrm{PL} 12$

24.50 GPNAM [1] SINE. 100 $\begin{array}{ll}0 & \% \\ 0 & 0 \\ 0 & 0 \\ 0 & 0\end{array}$ SINE. 100

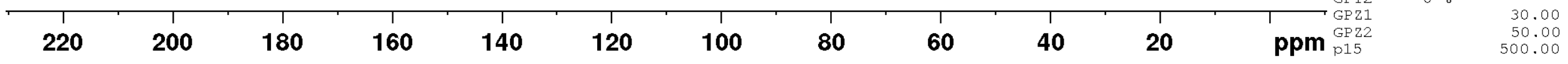



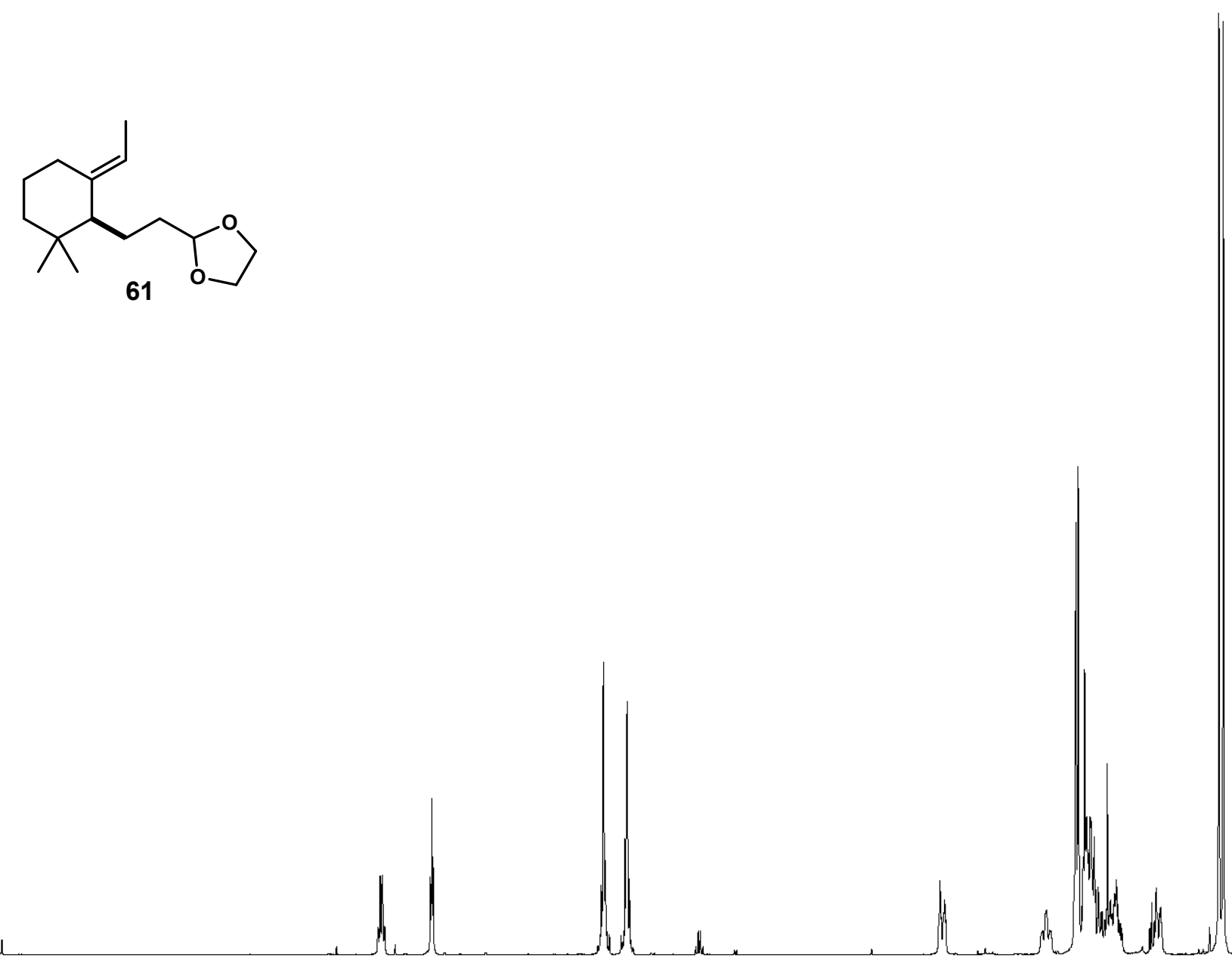

F2 - Acquisition Parame

$$
\text { Date }
$$

INSTRUM

PROBHD

PULPROG

TD

NS

DS

SWH
FIDRE
AQ

AQ
RG

RG
DW

DE

$\mathrm{TE}$
$\mathrm{D} 1$

$====$
NUC1

P1
PLW1

PLW1

F2 - Processing paramet

ramet

SF 600.1300341

WE

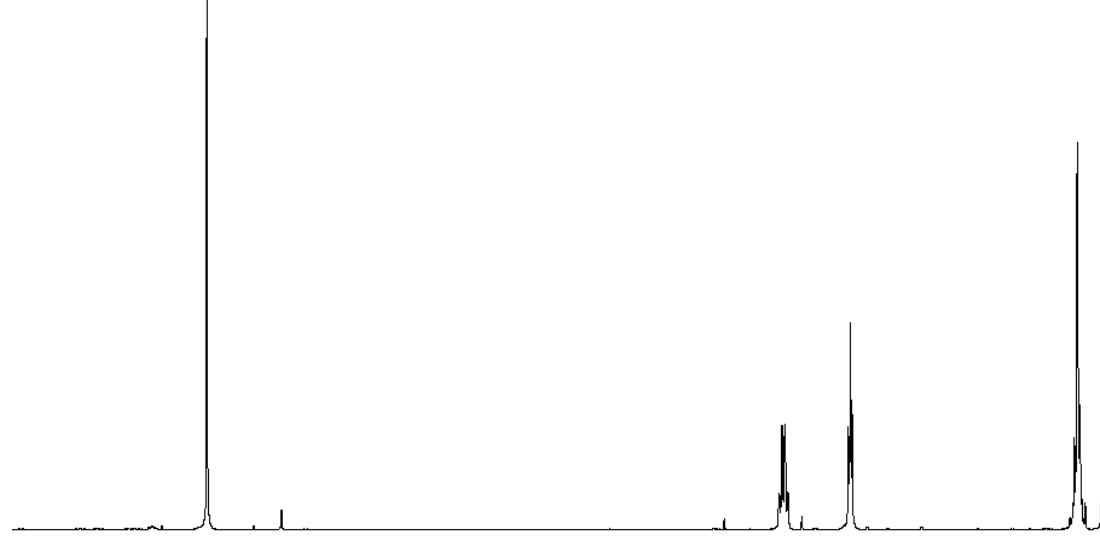
U

1.00

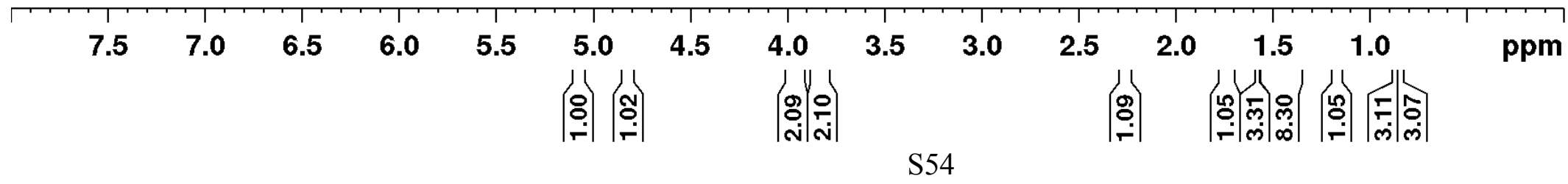




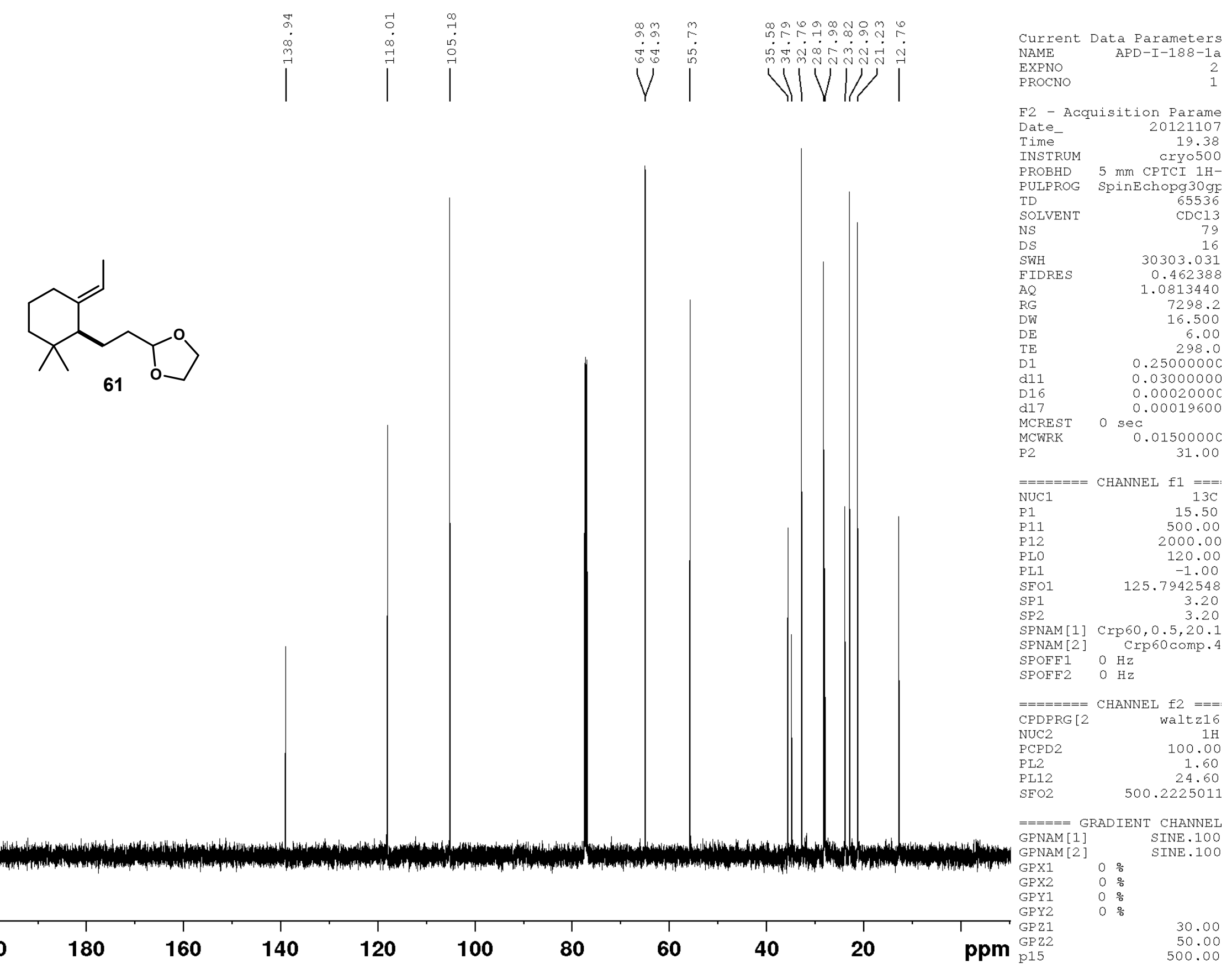




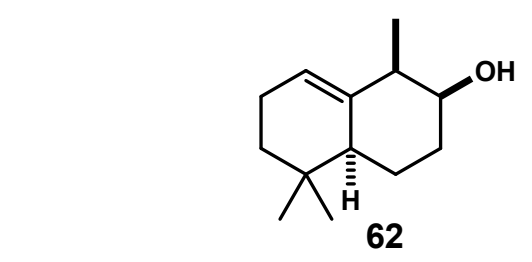

F2 - Acquisition Parame Date

Time

INSTRUM

PROBHD

PROBHD

PU

SOLVENT

NS

SWH

FIDRE

RG

DW

$\mathrm{DE}$

D1

SFO

SFO1
NUC1

20190809

15.07

$\mathrm{zg} 30$

$\mathrm{zg} 30$
98074

$\mathrm{DCl} 3$

P1
PLW1

9615.385

0.098042

5.0998478

18
52.000

13.70

$0.1000000 \mathrm{C}$

CHANNEL $\mathrm{f} 1===$

12.00
30.00000000

F2 - Processing paramet

SI

$\begin{array}{ll}\text { SF } & 600.1300349\end{array}$

0.30
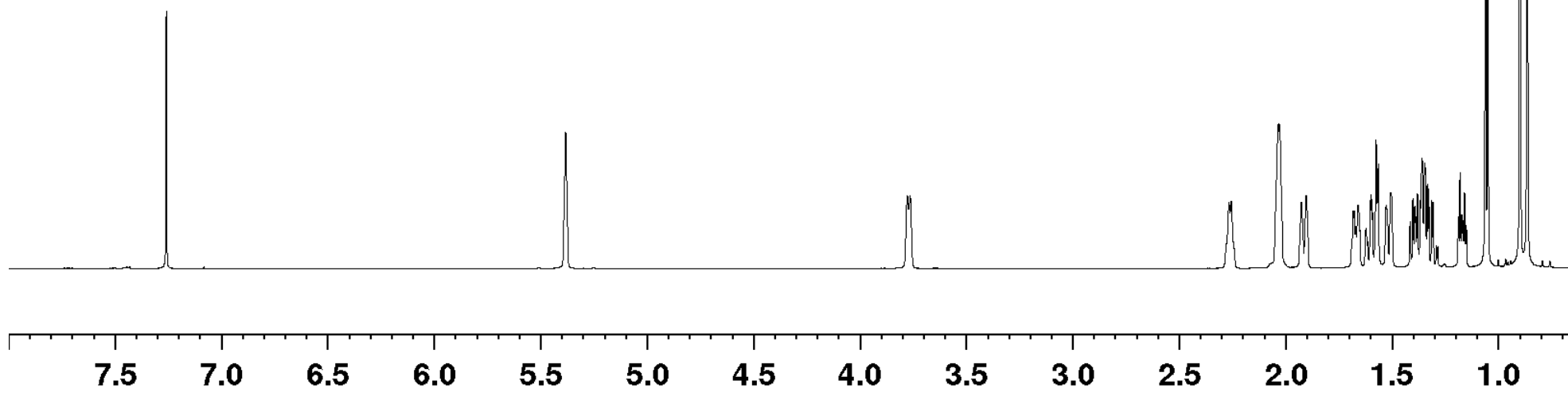

5.5
$\left|\begin{array}{l}8 \\ 0\end{array}\right|$
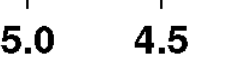

4.0
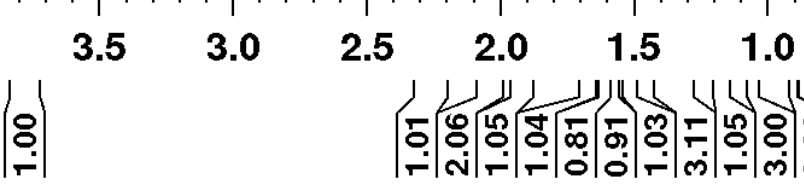

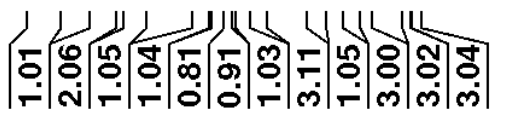

ppm

$\left|\begin{array}{c}0 \\ 0 \\ \hdashline\end{array}\right|$ 

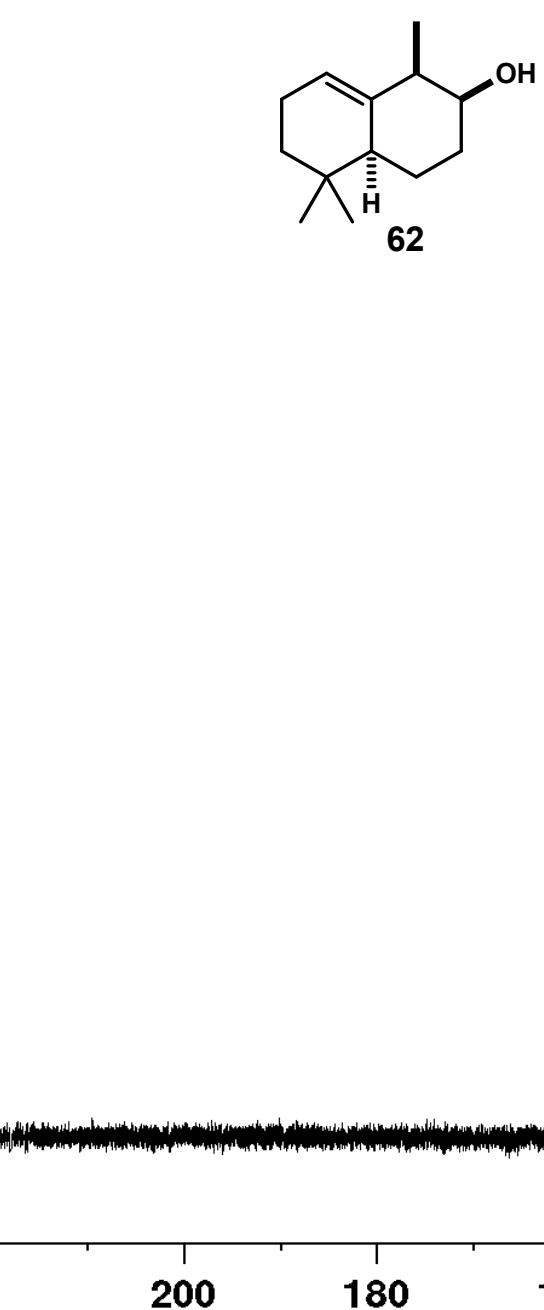

200 $\begin{array}{ll}0 & \vec{\sigma} \\ \dot{0} & \dot{\sigma} \\ \dot{0} & \overrightarrow{7} \\ \vec{r} & \mid\end{array}$

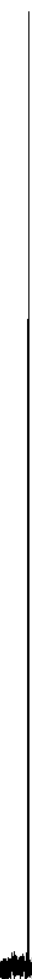

|

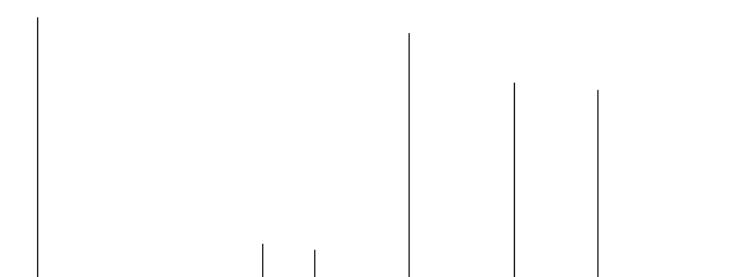$$
\text { (n) }
$$
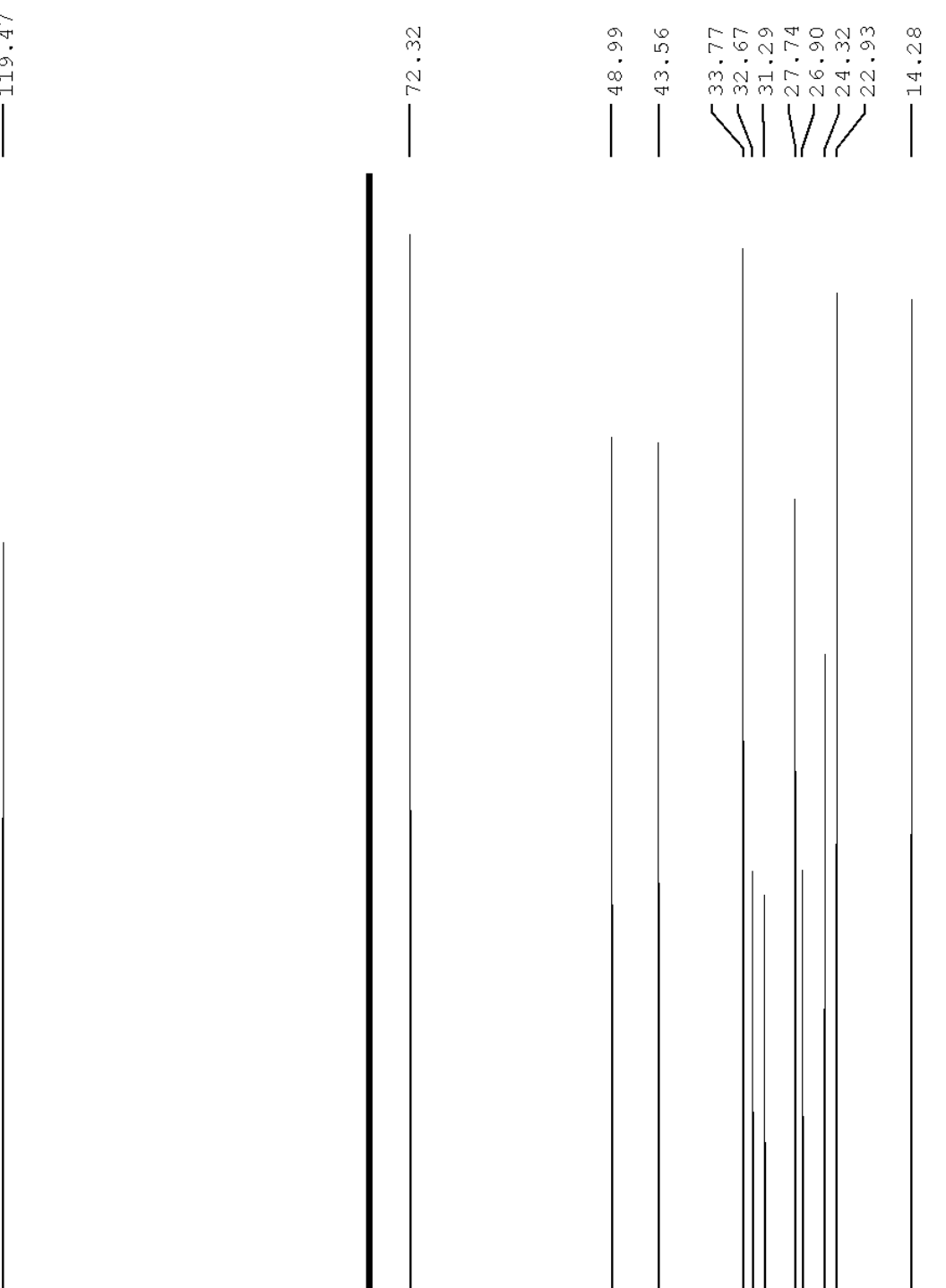

Current Data Parameters NAME 2-decalin alco

PROCNO

F2 - Acquisition Parame Date

Time

INSTRUM

PROBHD

TD

SOLVENT

NS

SWH

$\mathrm{AQ}$
$\mathrm{RG}$

DW

TE

D1

D11
TDO

$====$

SFO1

NUC1
P1

P1
PLW1

$==$

$\mathrm{SFO} 2$

CPDPRG [2

PCPD2

PLW 2

PIT 12

F2 - Processing paramet

SI 150.9025085

WD

LB

GB

PC

150.9028085

1.00

1.00 

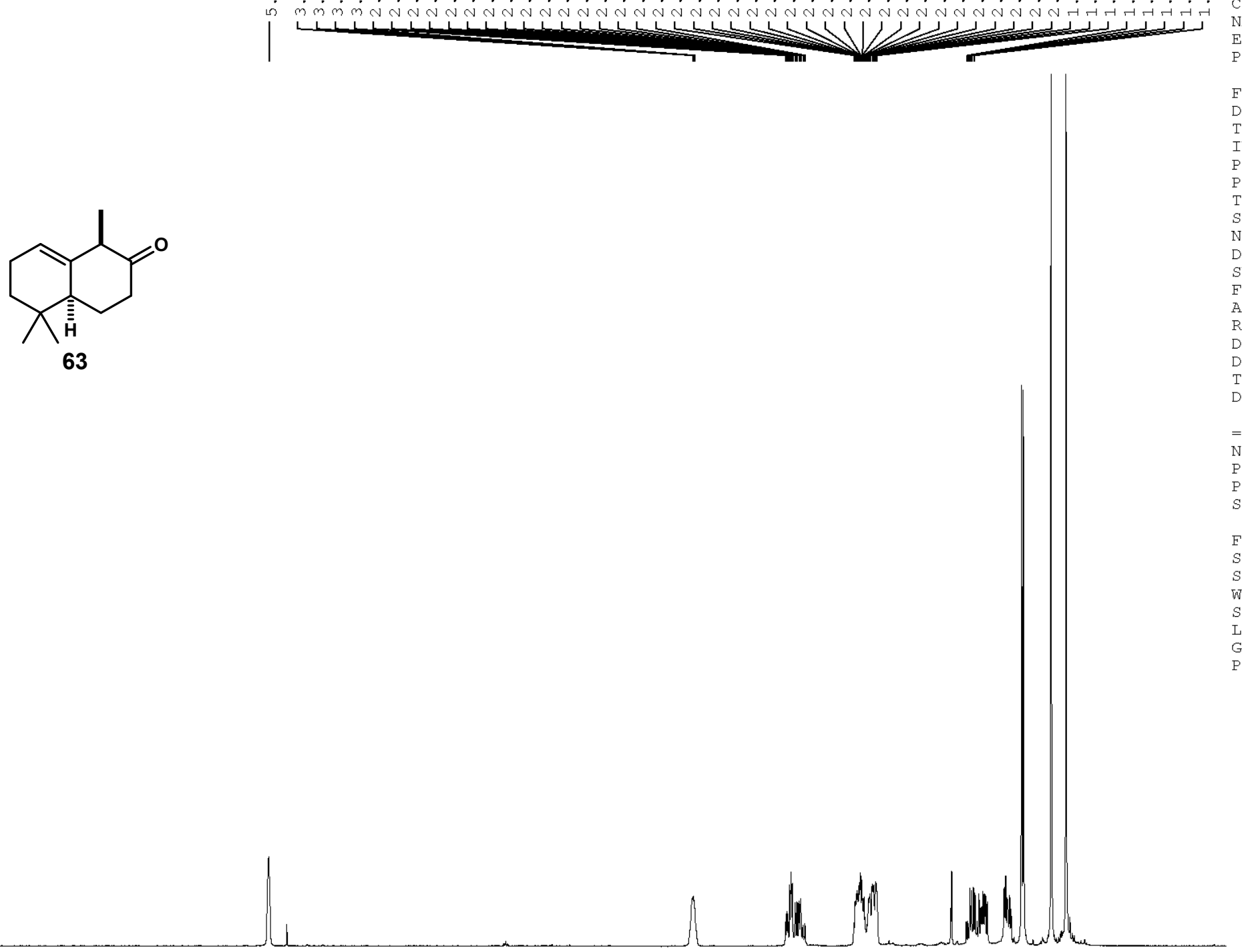

F2 - Acquisition Parame Date_ 20121214

98074

LVN

DS

2
2
9615.385

$\begin{array}{ll} & 9615.385 \\ & 0.098042\end{array}$

5.0998478

WW

63
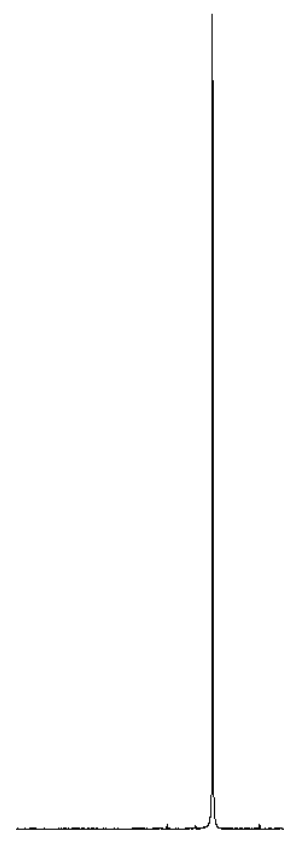

D1

812
52.000

6.00
298.1

$======$ CHANnEL fl $====$
NUC1
$1 \mathrm{H}$

8.00

23.01441956

F2 - Processing paramet

$\begin{array}{lr}\text { SI } & 65536 \\ \text { SF } & 600.1300347\end{array}$

0.30

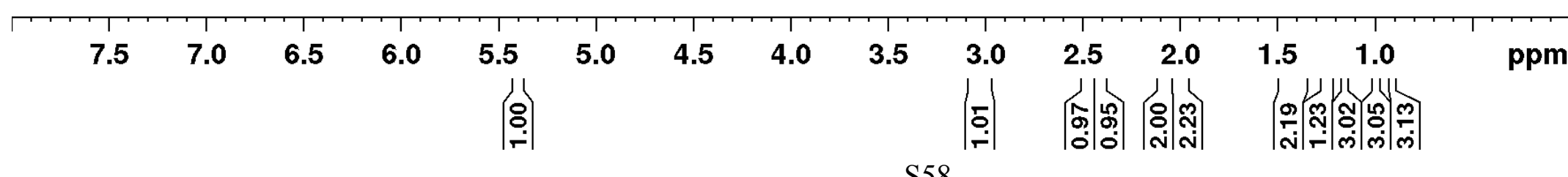




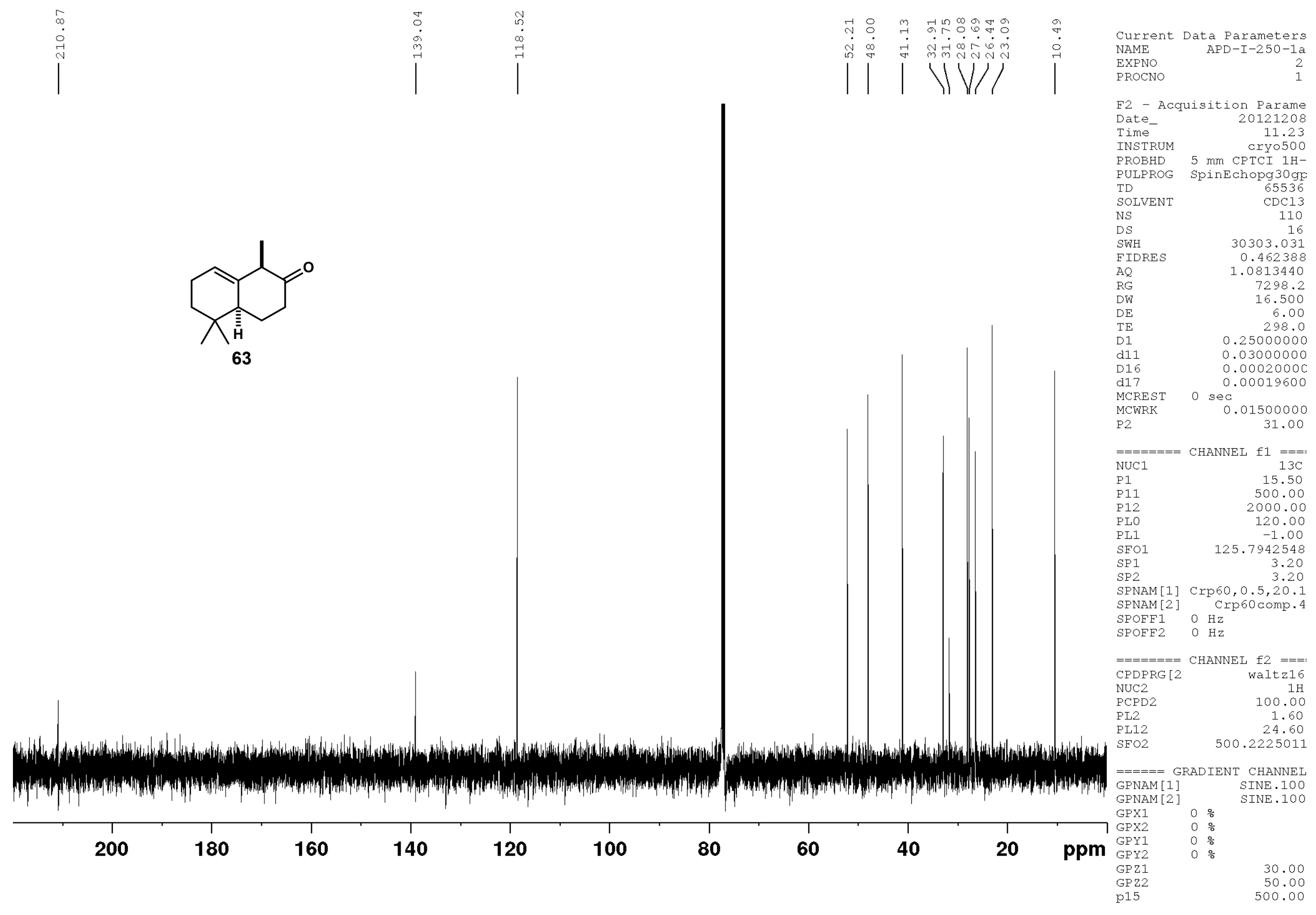



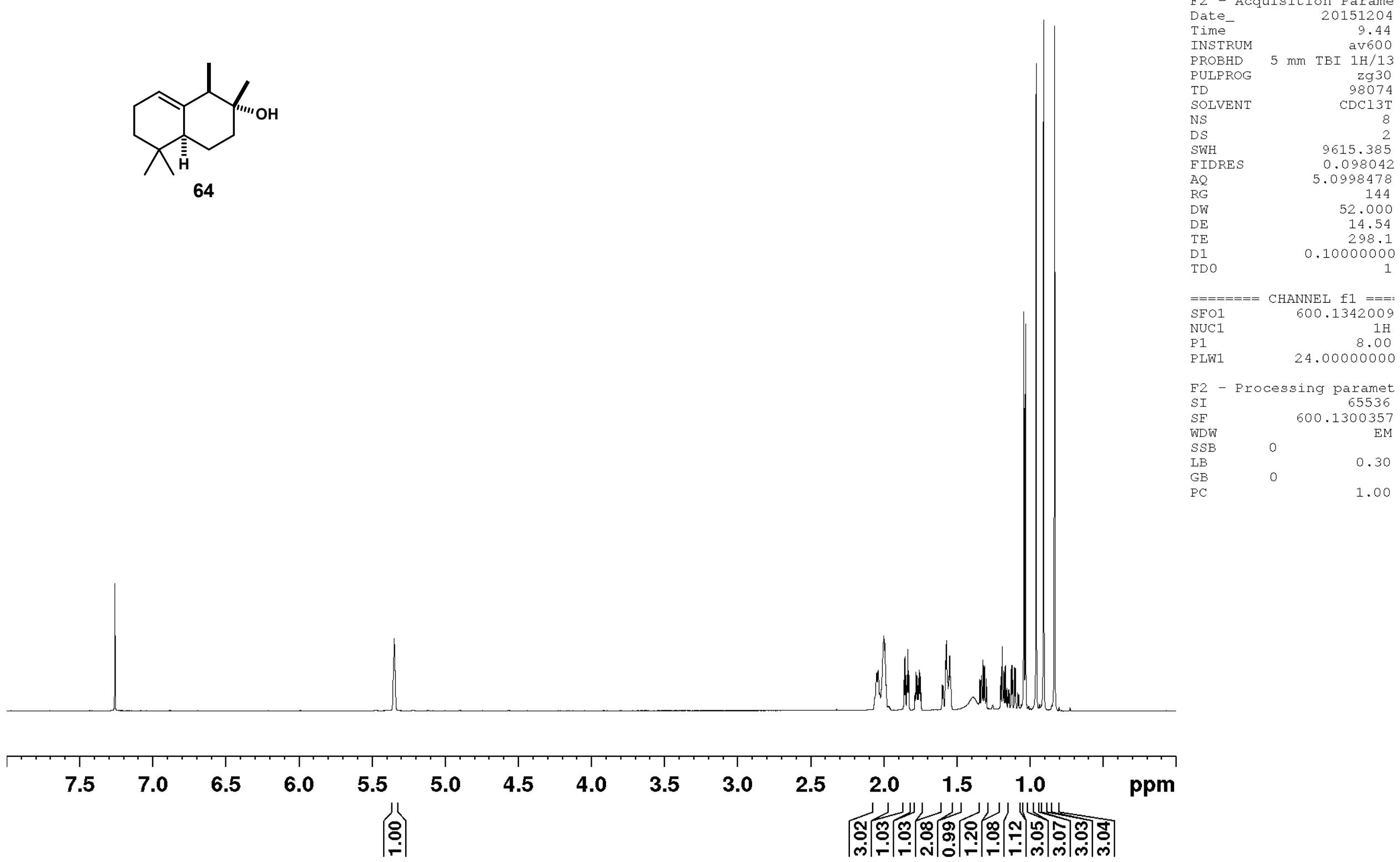


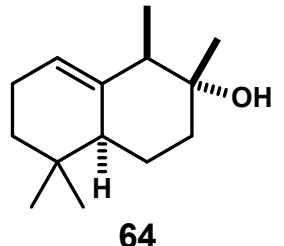

64
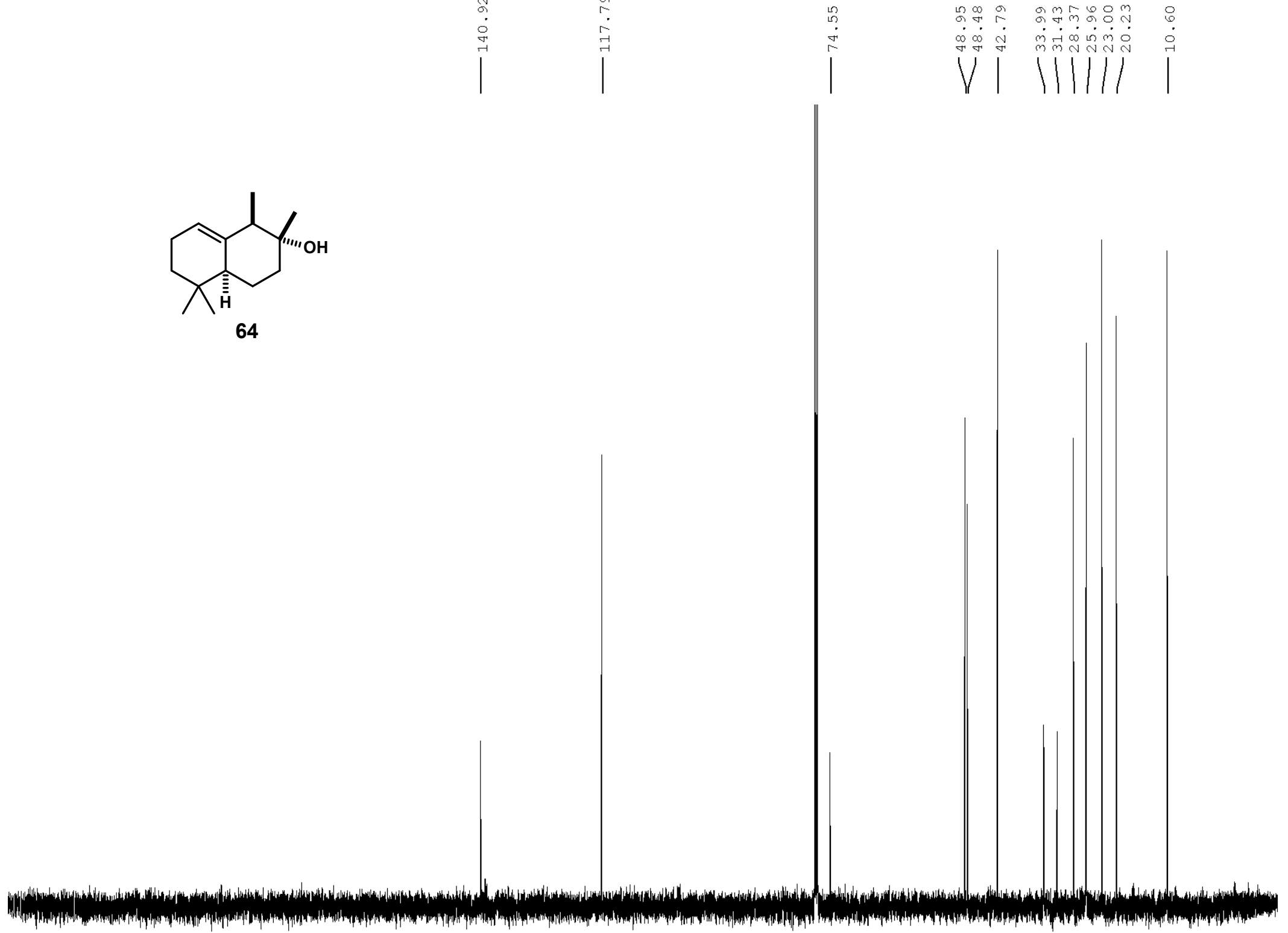

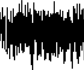

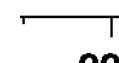

220
180

160

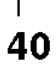

120
60

40 


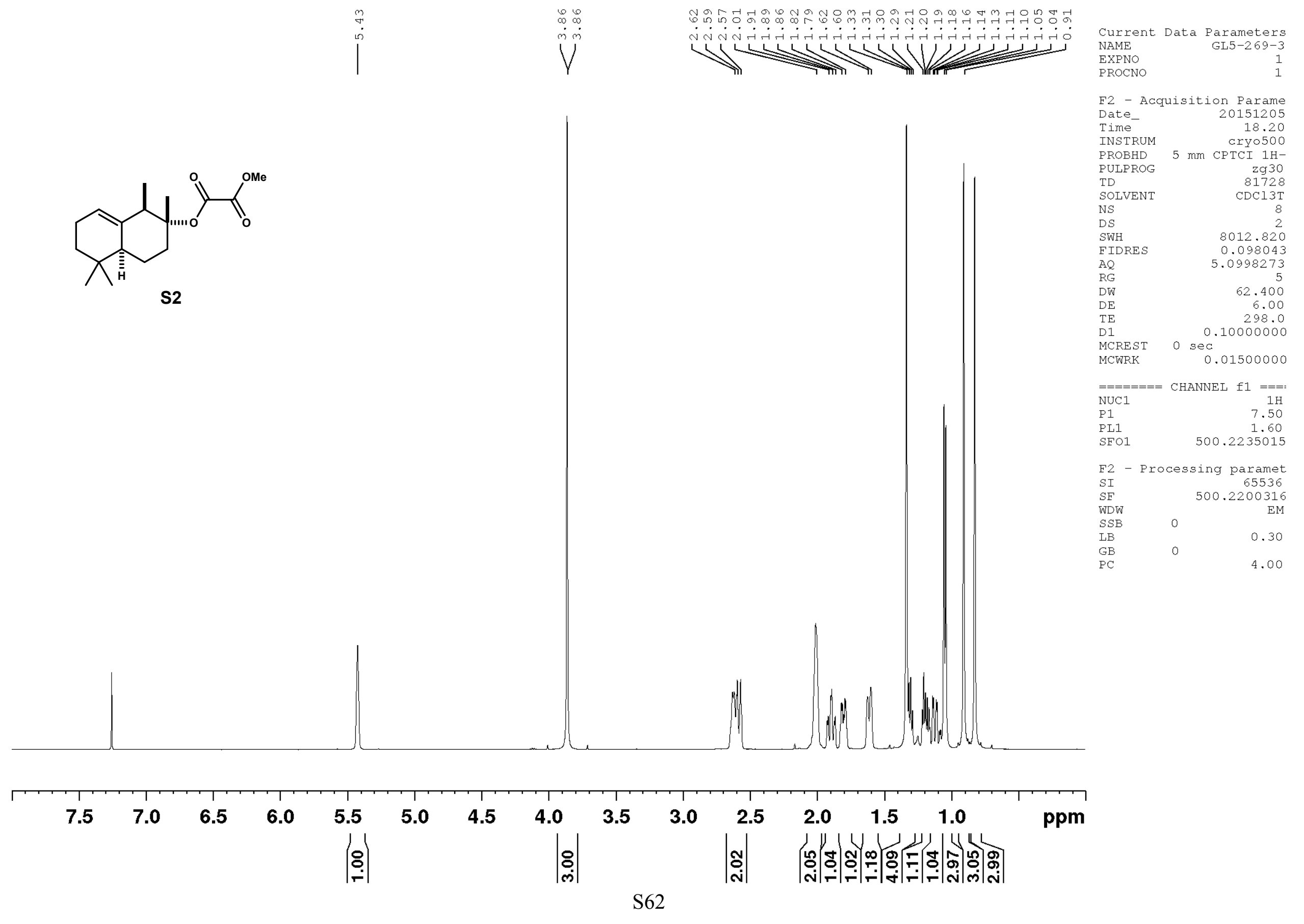




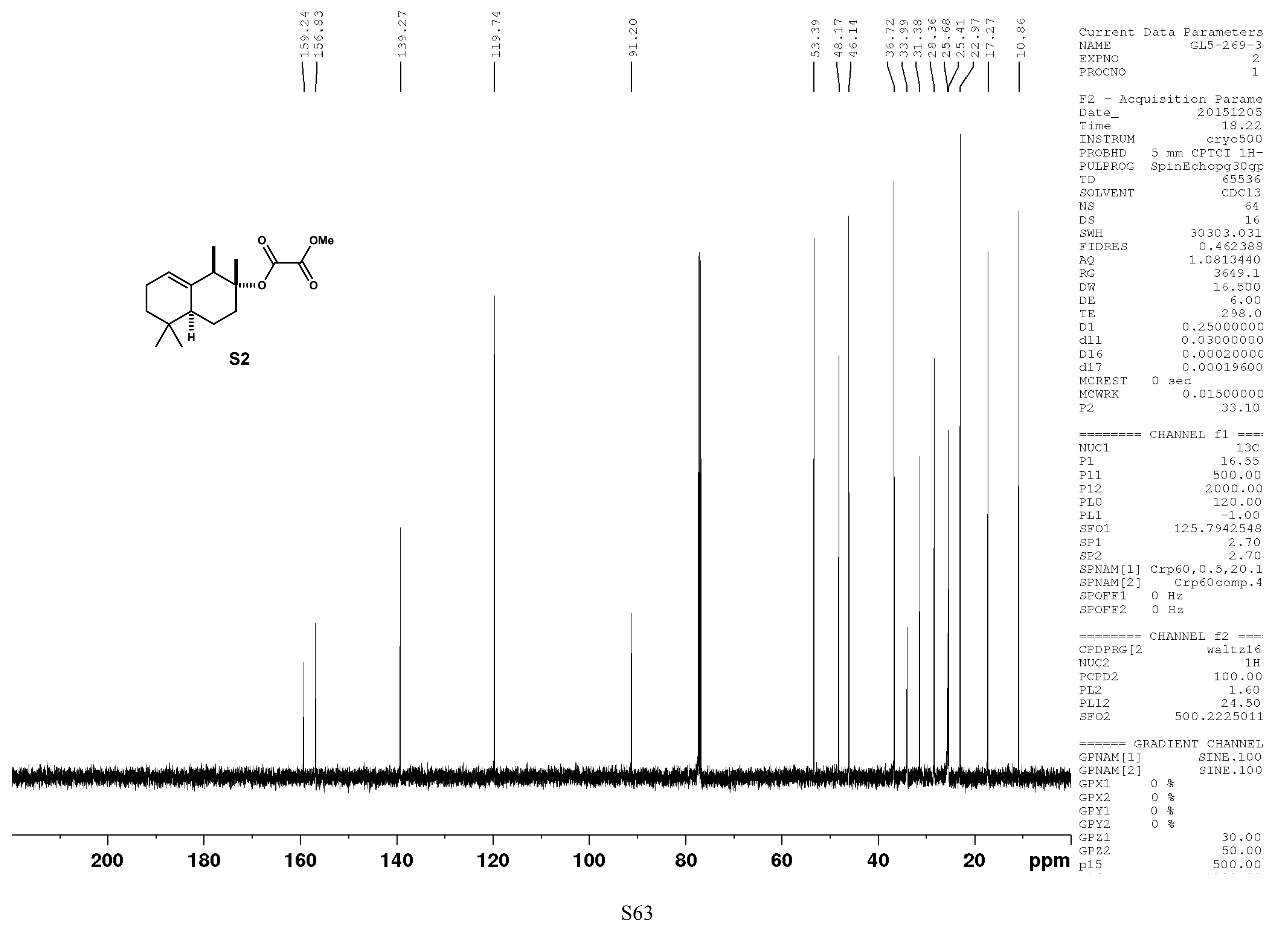




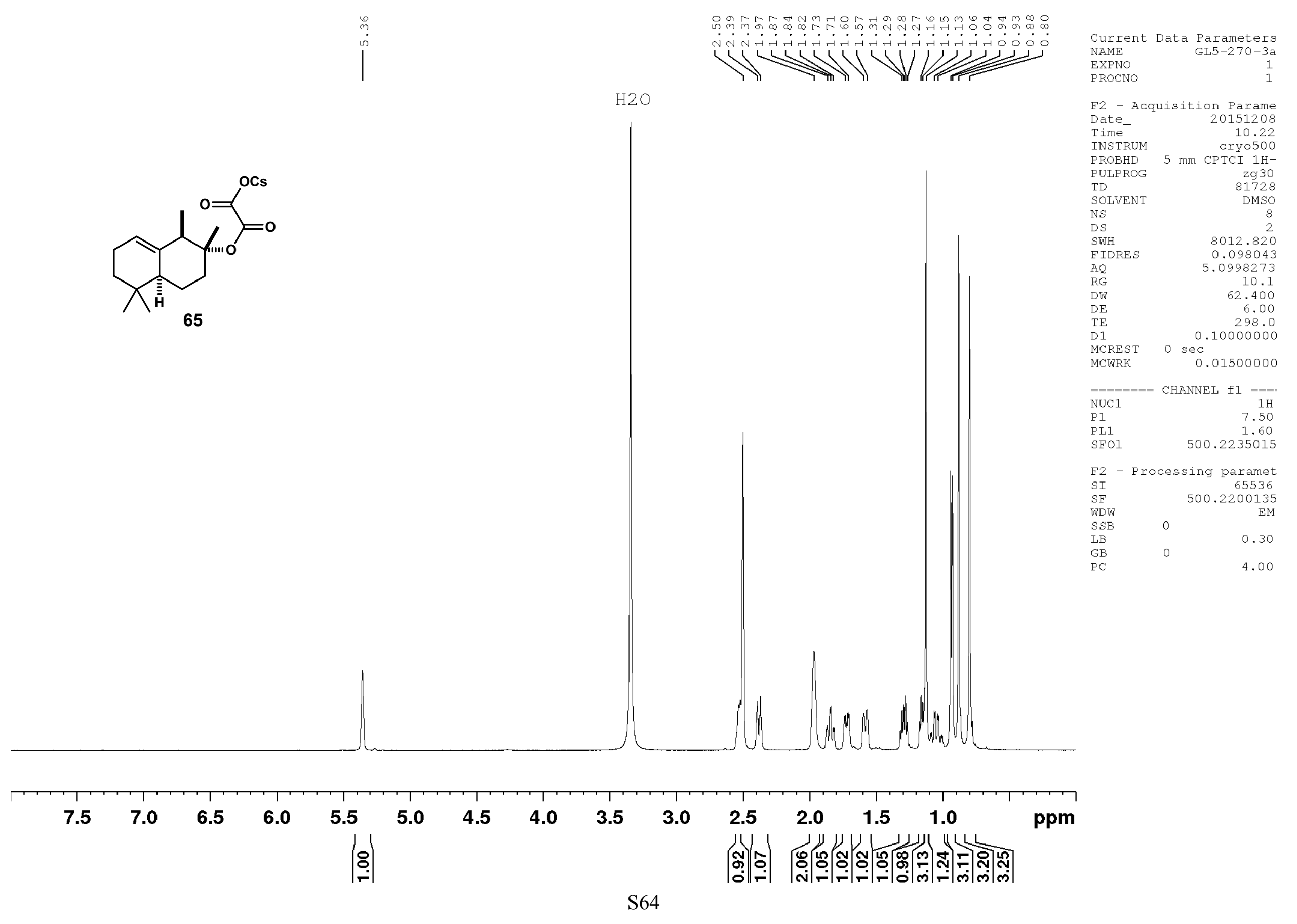




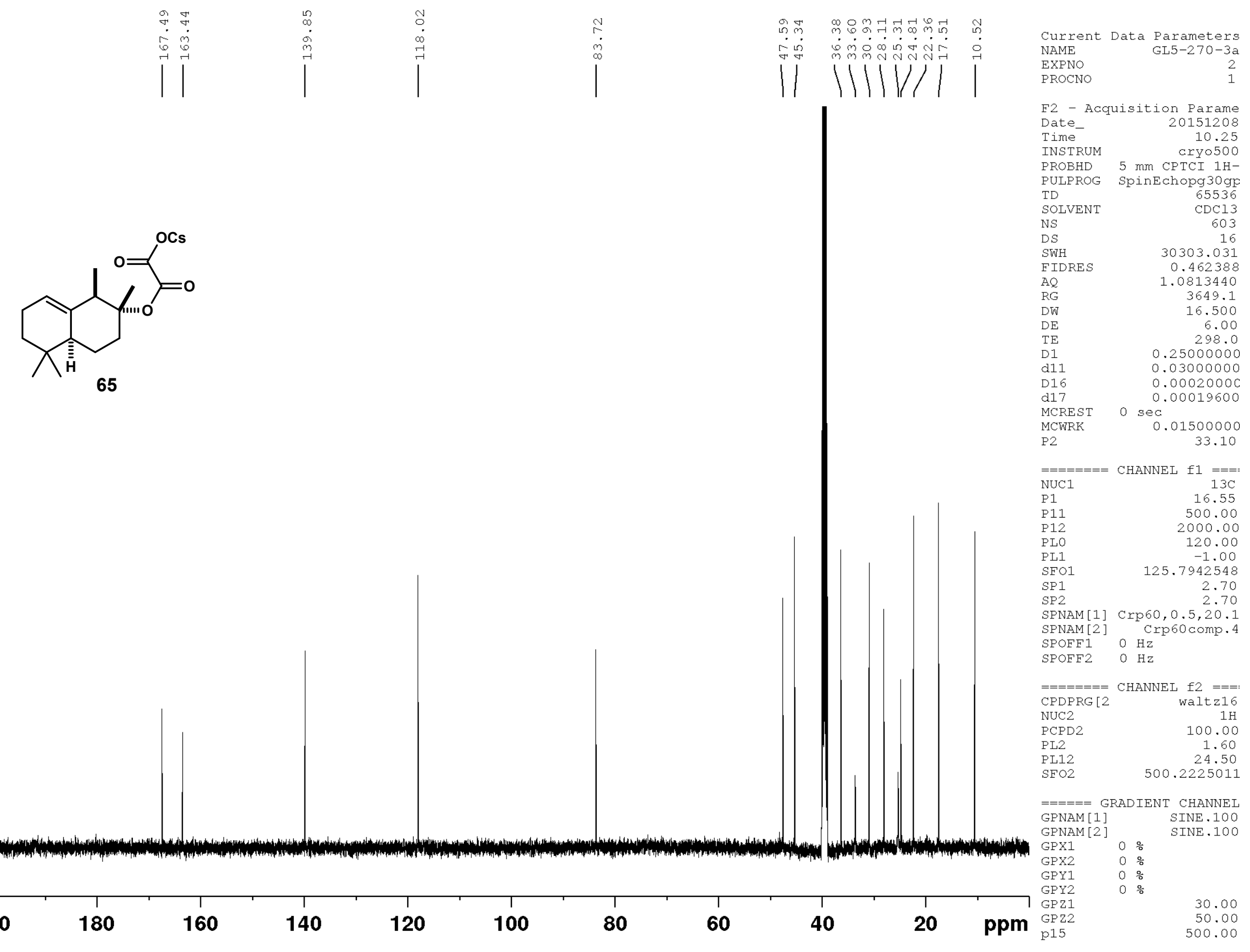




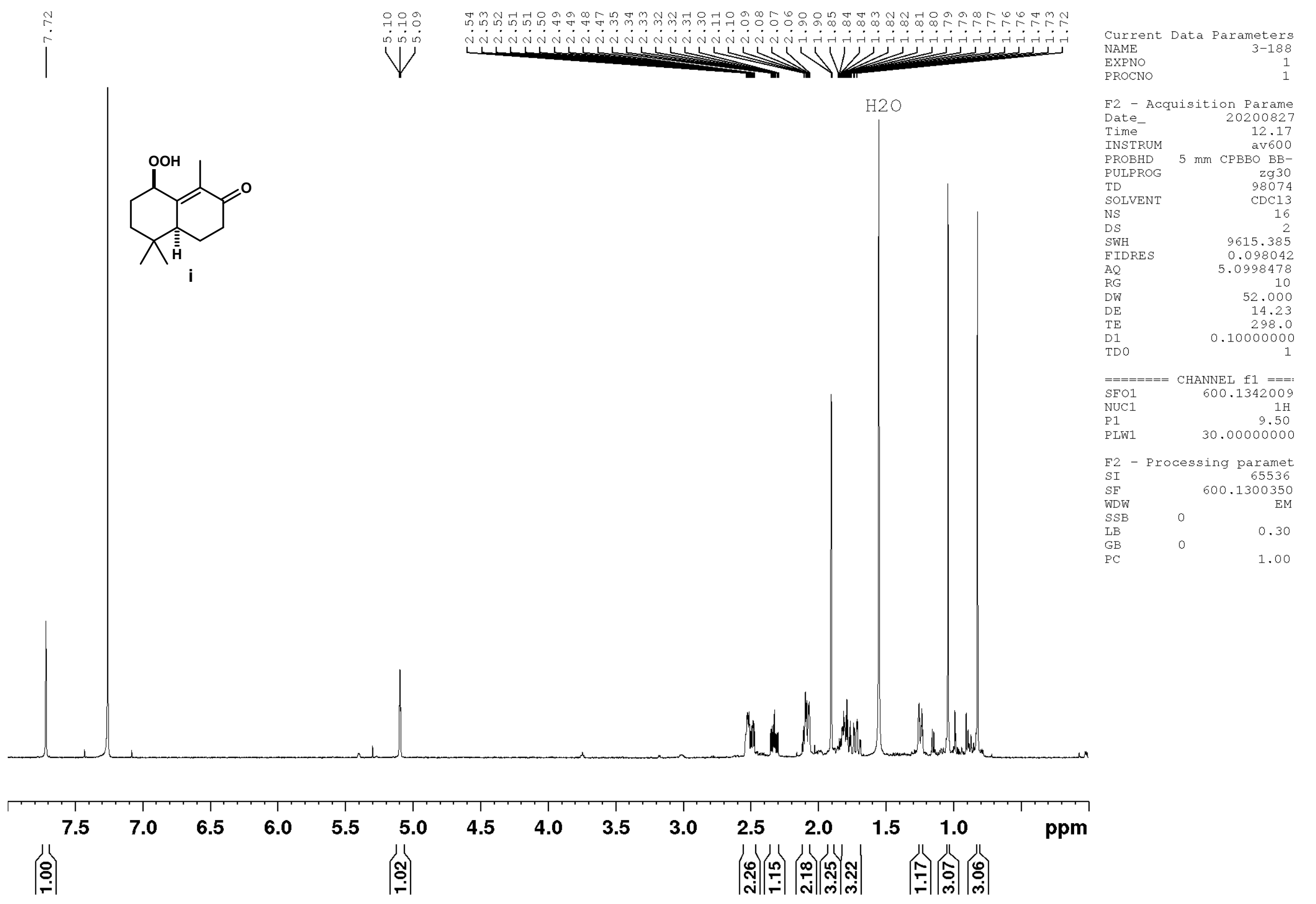




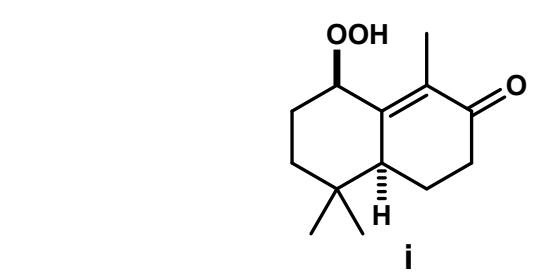

PULPROG SpinEchopg 30 ge

SOLVE

NS

$\begin{array}{lr}\text { DS } & 16 \\ \text { SWH } & 30303.031\end{array}$

FIDRES $\quad 0.462388$

$\begin{array}{lr}\text { RG } & 14596.5 \\ \text { DWT } & 16.500\end{array}$

16.500
$D E$

$\begin{array}{lr}\mathrm{TE} & 298.0 \\ \mathrm{D} 1 & 0.25000000\end{array}$

d11 $\quad 0.03000000$

D16 0.000

d17 $\quad 0.00019600$

MCREST

MCWR

sec

0.01500000

31.00

$=======$ CHANNEL f1 $====$
NUC1 $13 \mathrm{C}$
P1

$\begin{array}{lr}\text { P1 } & 15.50 \\ P 11 & 500.00\end{array}$

500.00

PLO $\quad 120.00$

PL1 125.7942548

$\begin{array}{ll}\mathrm{SFO} & 125.7942548\end{array}$

$\begin{array}{lr}\text { SP1 } & 125.7942548 \\ \text { SP2 } & 3.20 \\ \text { SPEM [1] CrP60 } & 3.20\end{array}$

SPNAM [1] Crp60, 0.5,20.1 SPNAM [2] Crp60comp.4

$\begin{array}{lll}\text { SPOFF1 } & 0 & \mathrm{~Hz} \\ \text { SPOFF2 } & 0 & \mathrm{~Hz}\end{array}$

$=======$ CHANNEL $f 2===$
CPDPRG $[2 \quad$ waltz16 NUC2 PCPD2 100.00

$\mathrm{PL} 12$

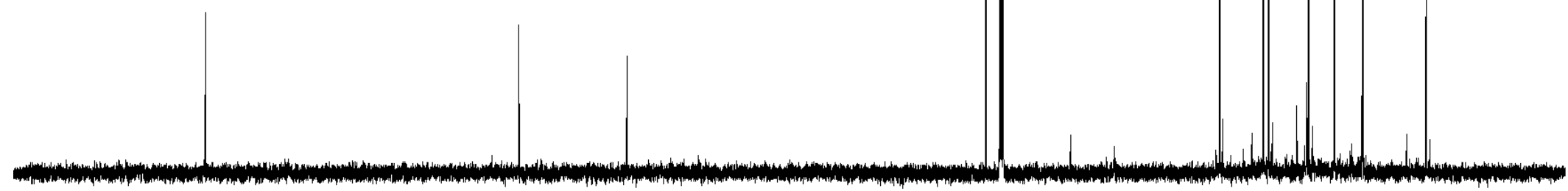
GPNAM [1] SINE. 100

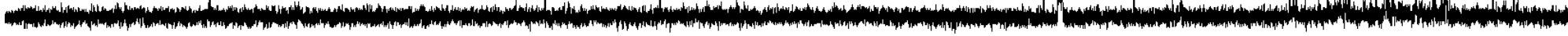
GPX2

GPY1 


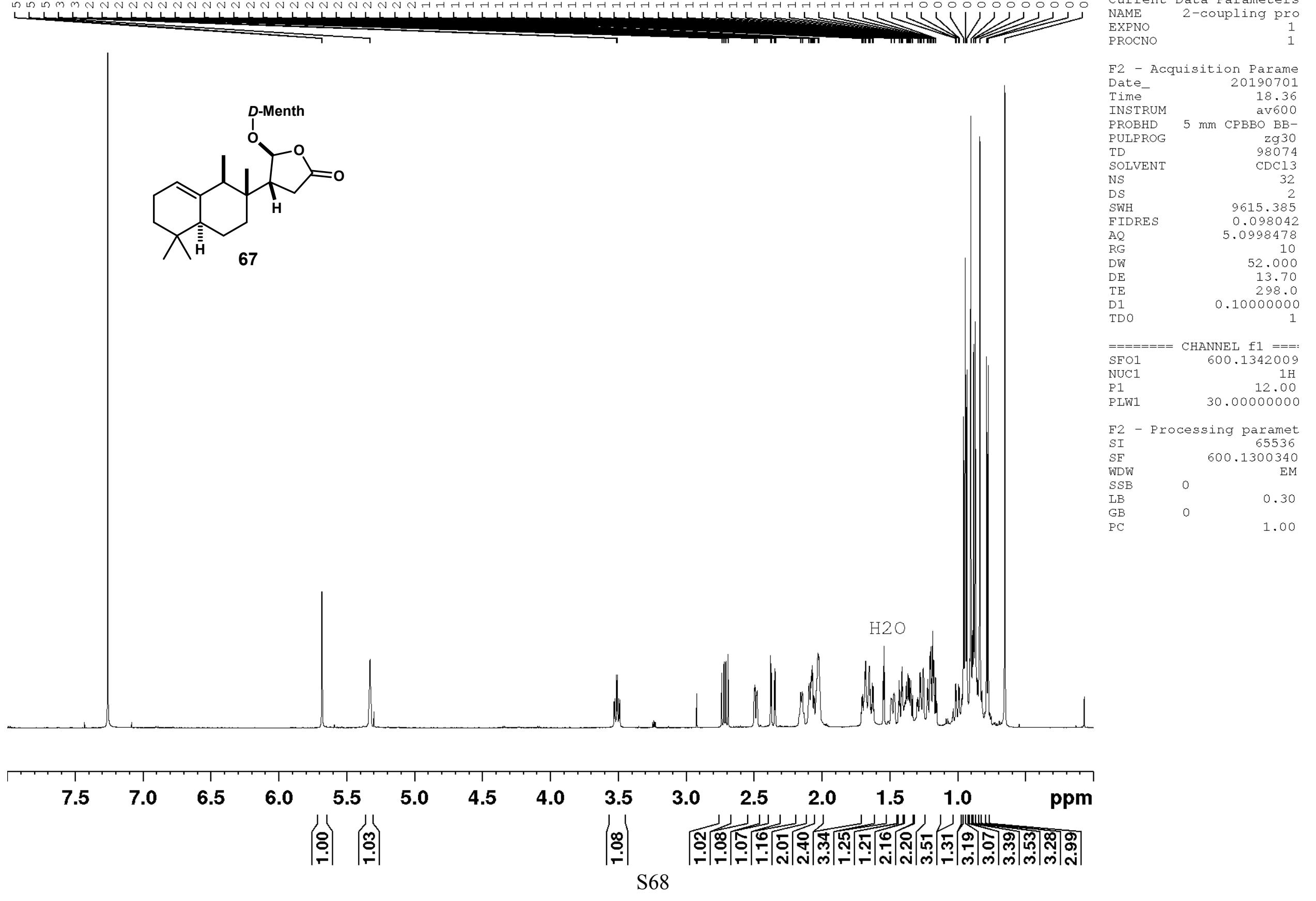




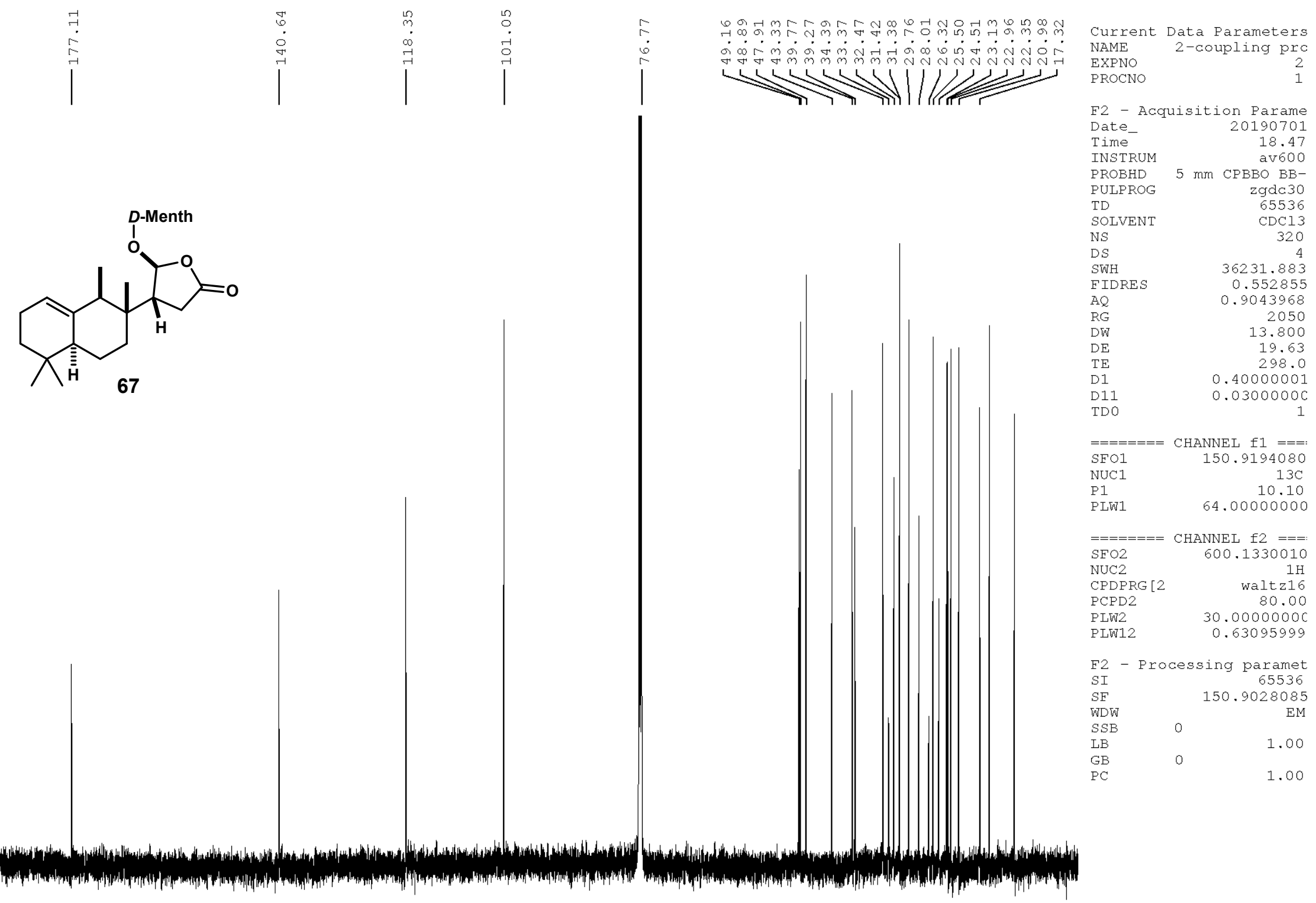

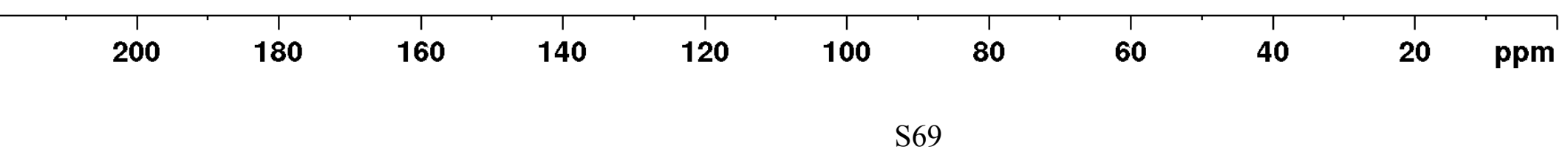




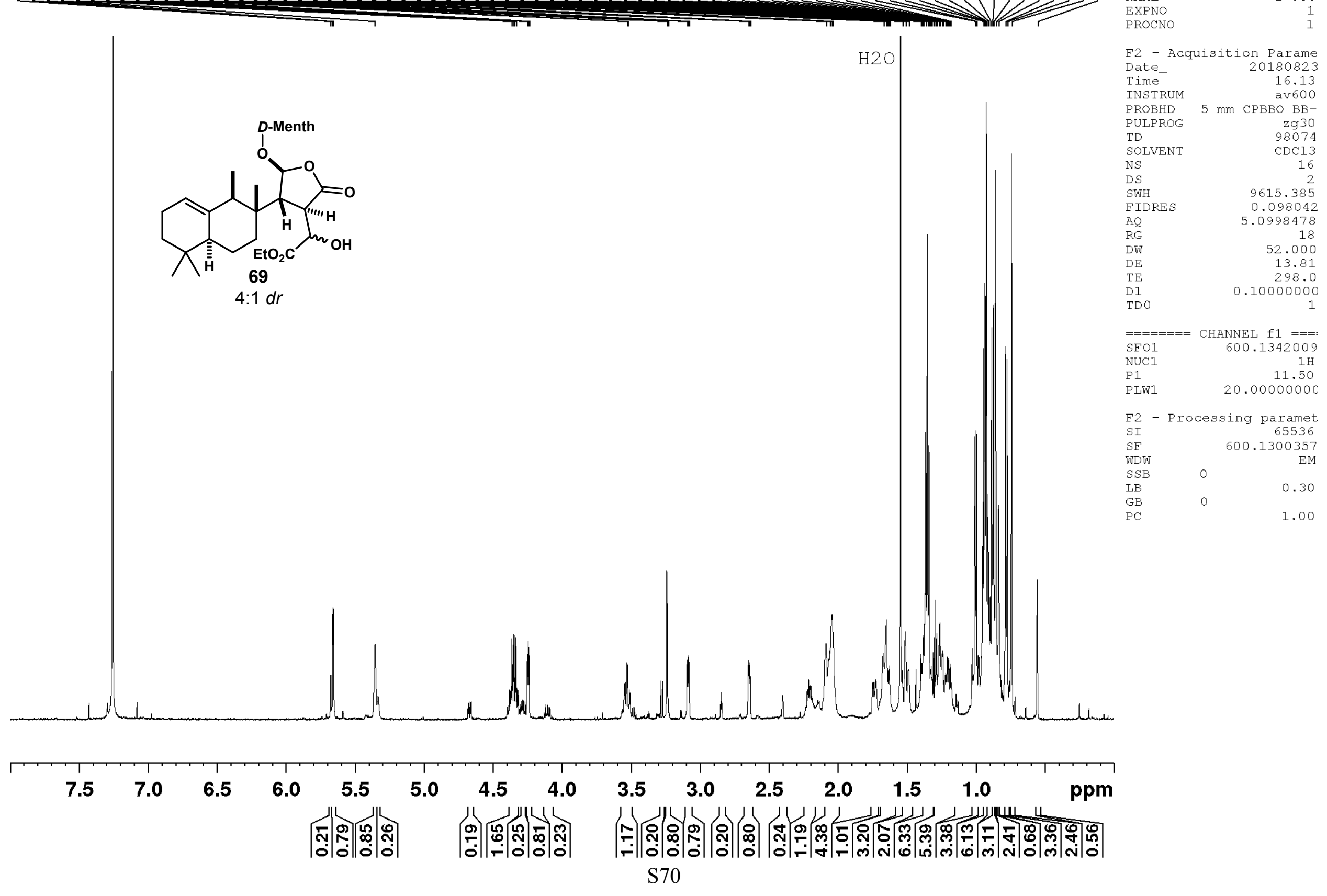




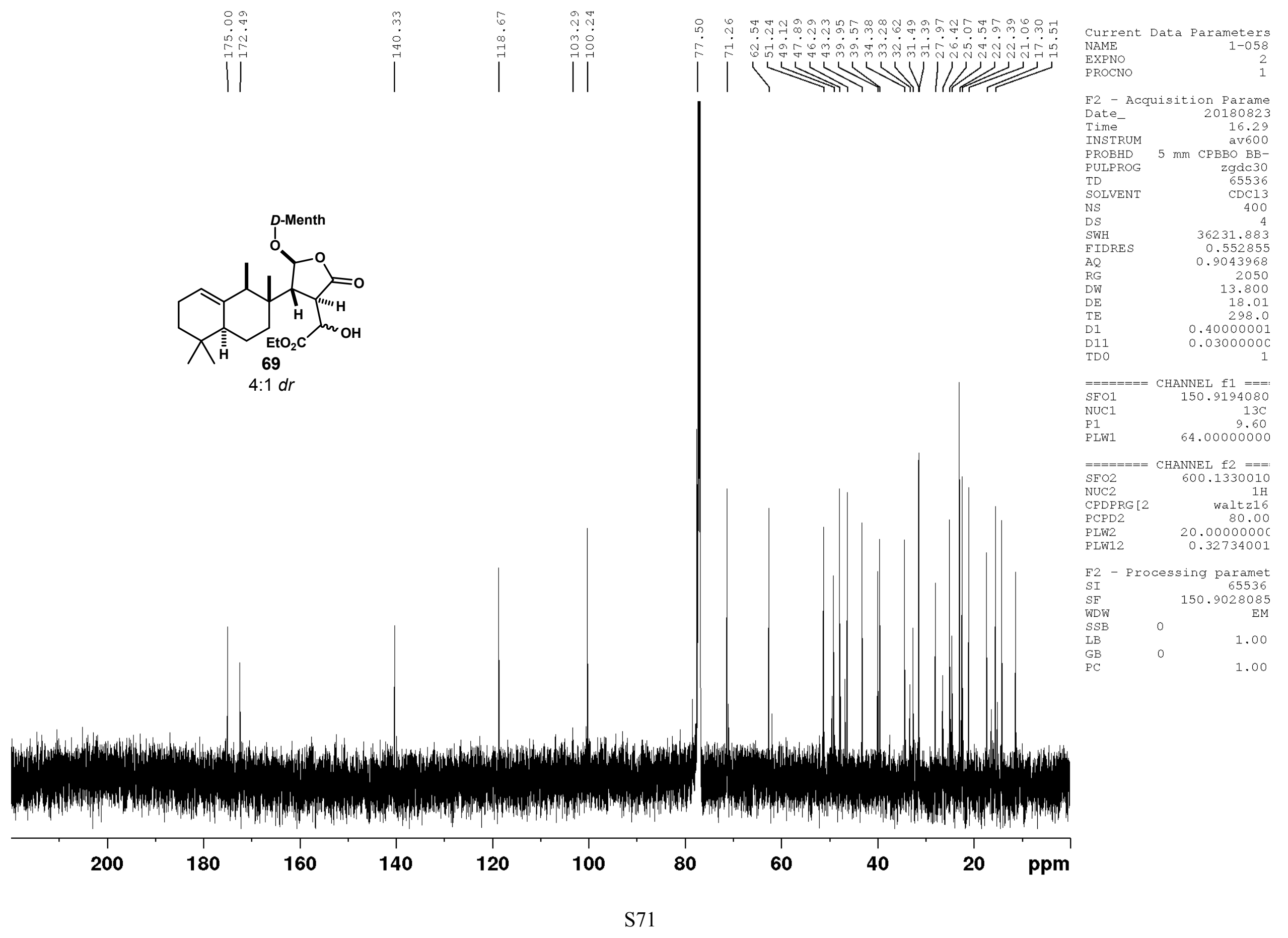



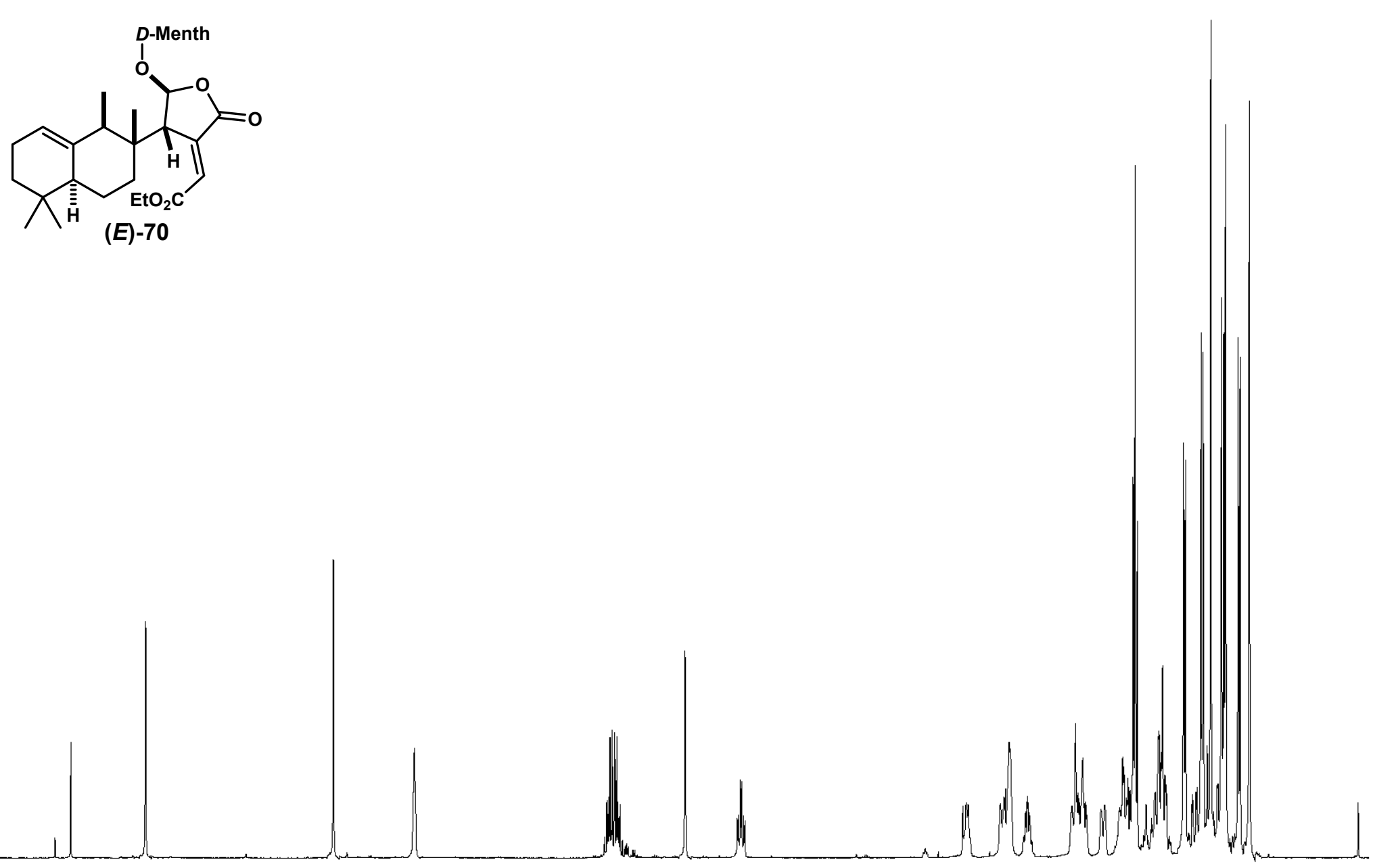

F2 - Acquisition Parame

Date__ 20180601

INSTRUM

PROBHD

PULPROG

TD

SOLVENT

DS

SWH

FID

$(E)-70$

DW

$\mathrm{DE}$

D1

13.49
av600

mm $\mathrm{CPBBO}$ BB-

zg30

$\mathrm{CDCl} 3$

$=======$ CHANNEL $\mathrm{f} 1===$ :

$\begin{array}{rr}\text { SFO1 } & 600.1342009 \\ \text { NUC1 } & 1 \mathrm{H}\end{array}$

PLW1 $20.0000000 \mathrm{C}$

F2 - Processing paramet

SI 65536

$\begin{array}{ll}\text { SF } & 600.1300342\end{array}$

0.30

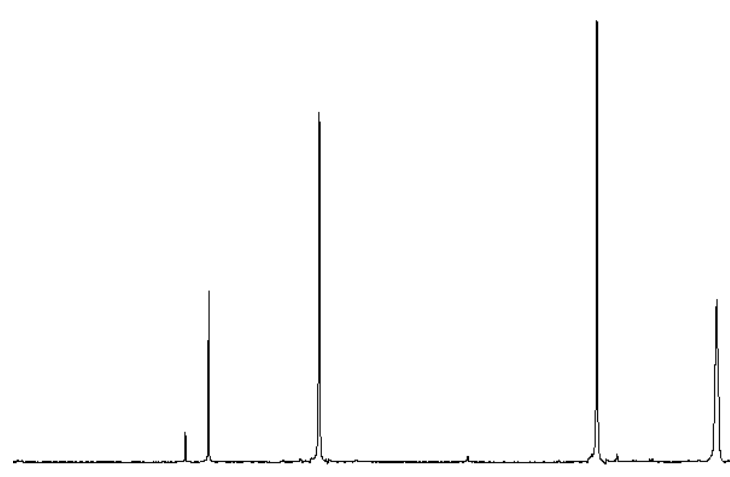

$\mathrm{PC}$

1.00

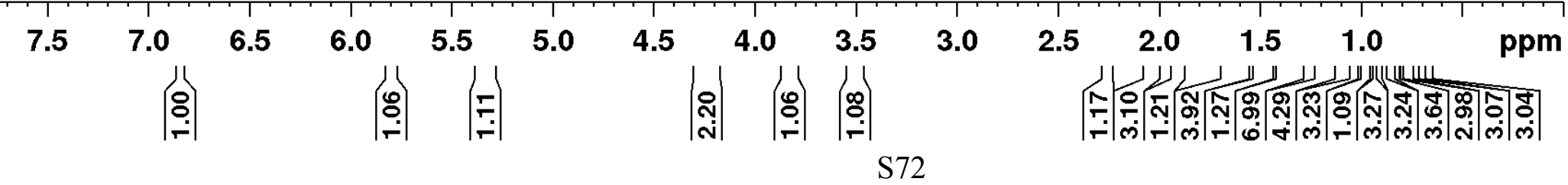




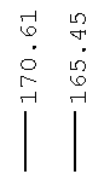 \\ |}

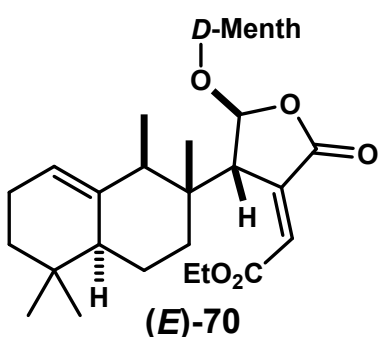

$(E)-70$

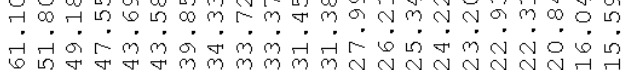

Yw W W

NAME

EXPNO
PROCNO

F2 - Acquisition Parame$$
\text { Date }
$$

INSTRUM

PROBHD

PULPROG

TD

SOLVENT

NS

SWH

FIDRES

$\mathrm{AQ}$

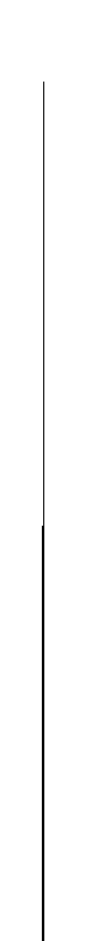

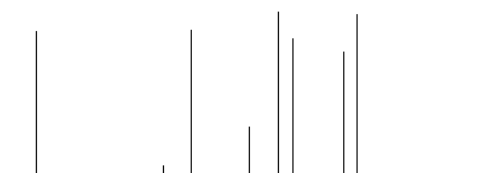

$\mathrm{RW}$
$\mathrm{DE}$

DE

D1

D11

SFO

P1

PLW1

$====$
SFO2
NUC2

CPDPRG [

PCPD2
PLW2

PLW12

F2 - Processing paramet

SI

SF

WDW

$\mathrm{LB}$
$\mathrm{GB}$ $1 \mathrm{H}$
600.1330010
Parameters 2 20180601 zgdc 30 65536 $\mathrm{CDCl} 3$ 36231.883
0.552855 0.552855 0.9043968 2050
13.800 18.01 0.40000001 .03000000 CHANNEI $f 1===$ 150.9194080
$13 \mathrm{C}$ 64.00000000 CHANNEL $\mathrm{f2}===$ waltz16 $20.0000000 \mathrm{C}$ .32734001 65536 150.9028085
EM 0 1.00 1.00 

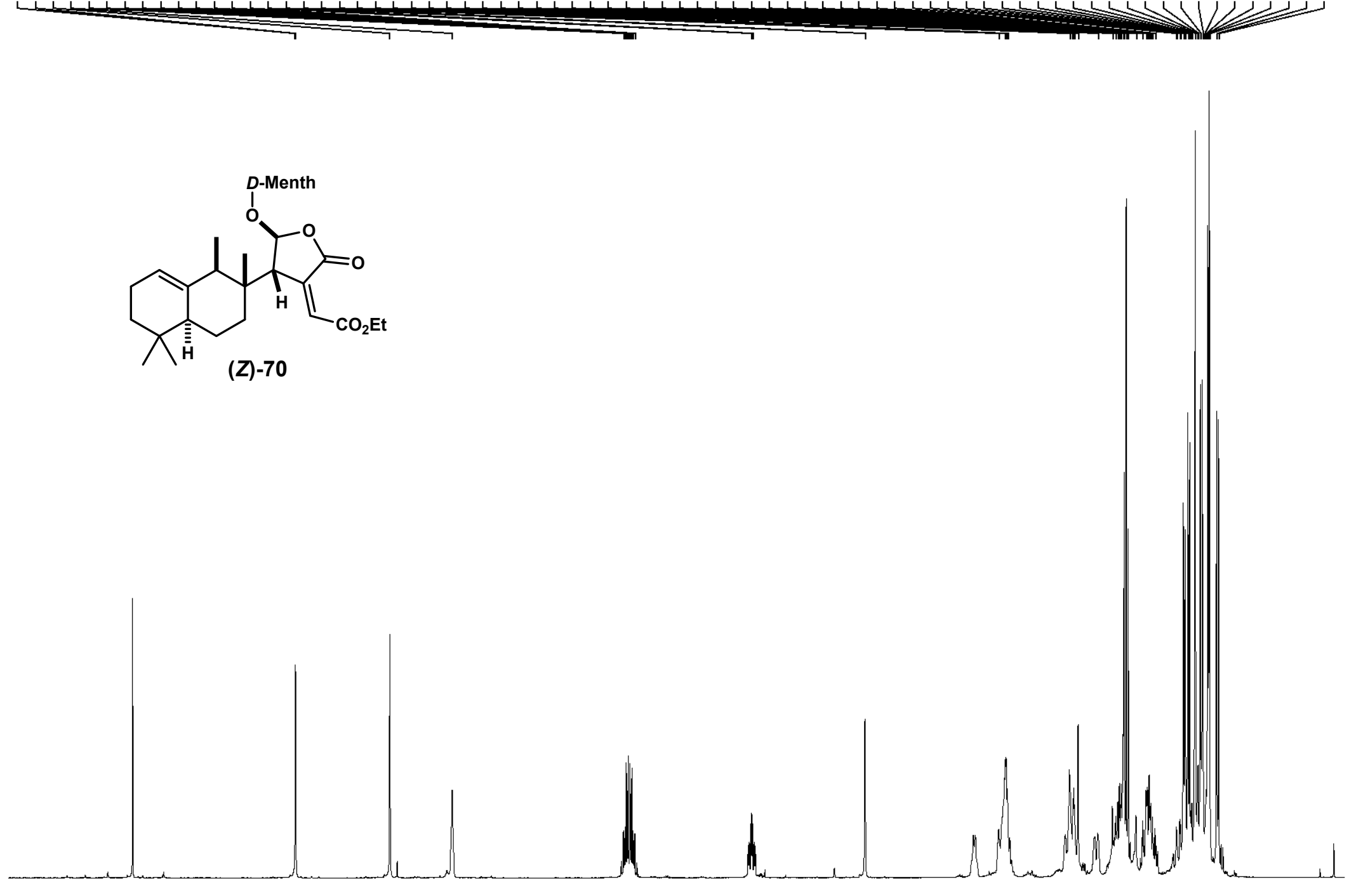

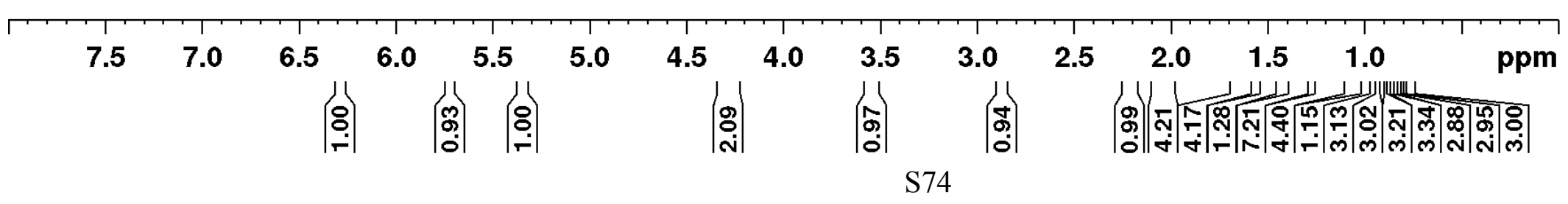




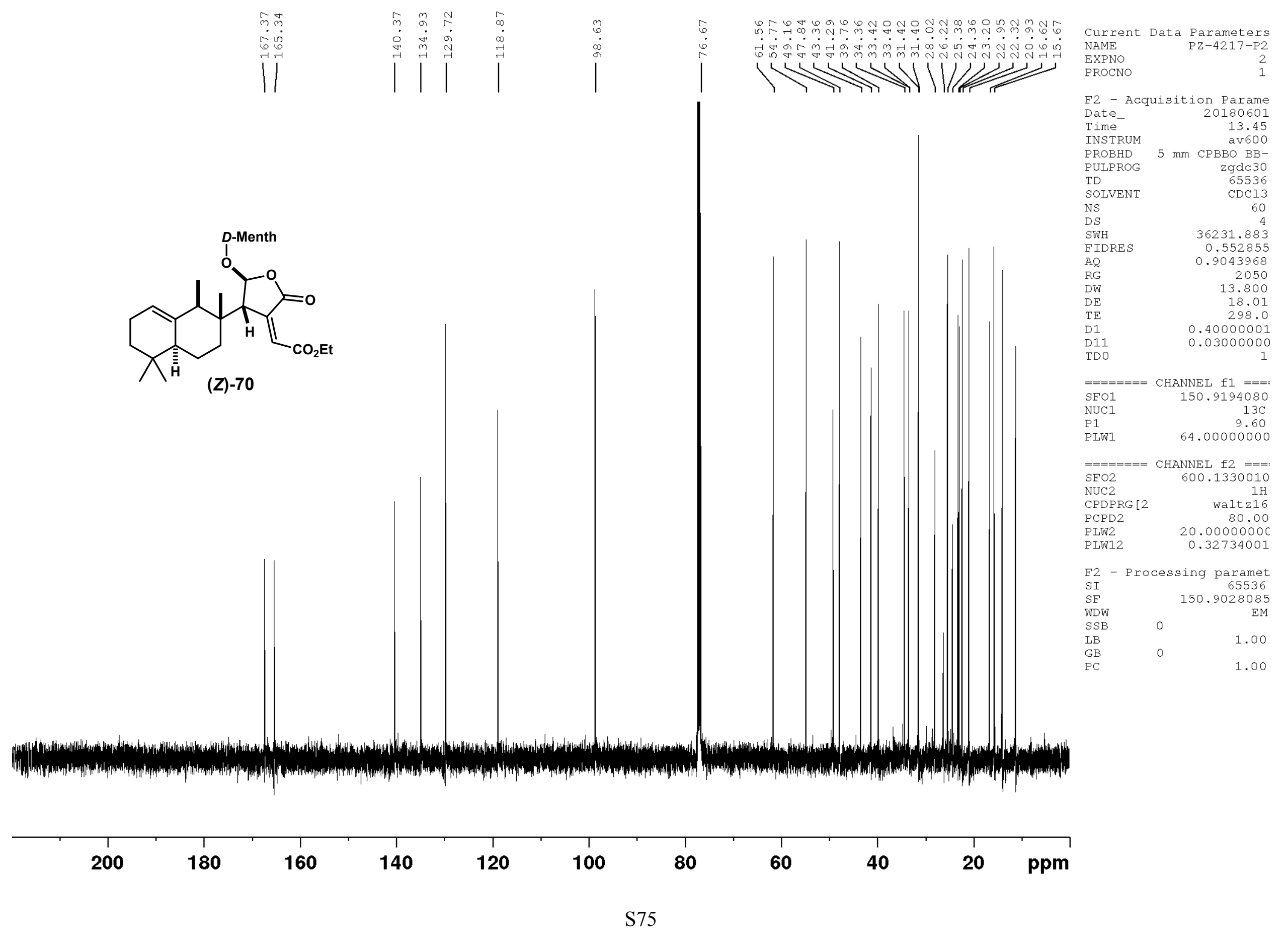




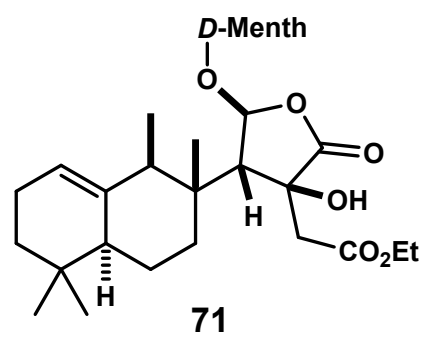

F2 - Acquisition Parame Date__ 20190625 Time INSTRUM PROBHD $5 \mathrm{~mm}$ CPBBO BBPULPROG TD

SOLVENT

NS

DS

SWH
FIDRES

$\mathrm{AQ}$

RG

$\mathrm{DW}$

$\mathrm{TE}$
$\mathrm{D} 1$

D1
TDO

98074

$\mathrm{CDCl} 3$
48
2

9615.385 5.0998478 10 13.70 0.10000000
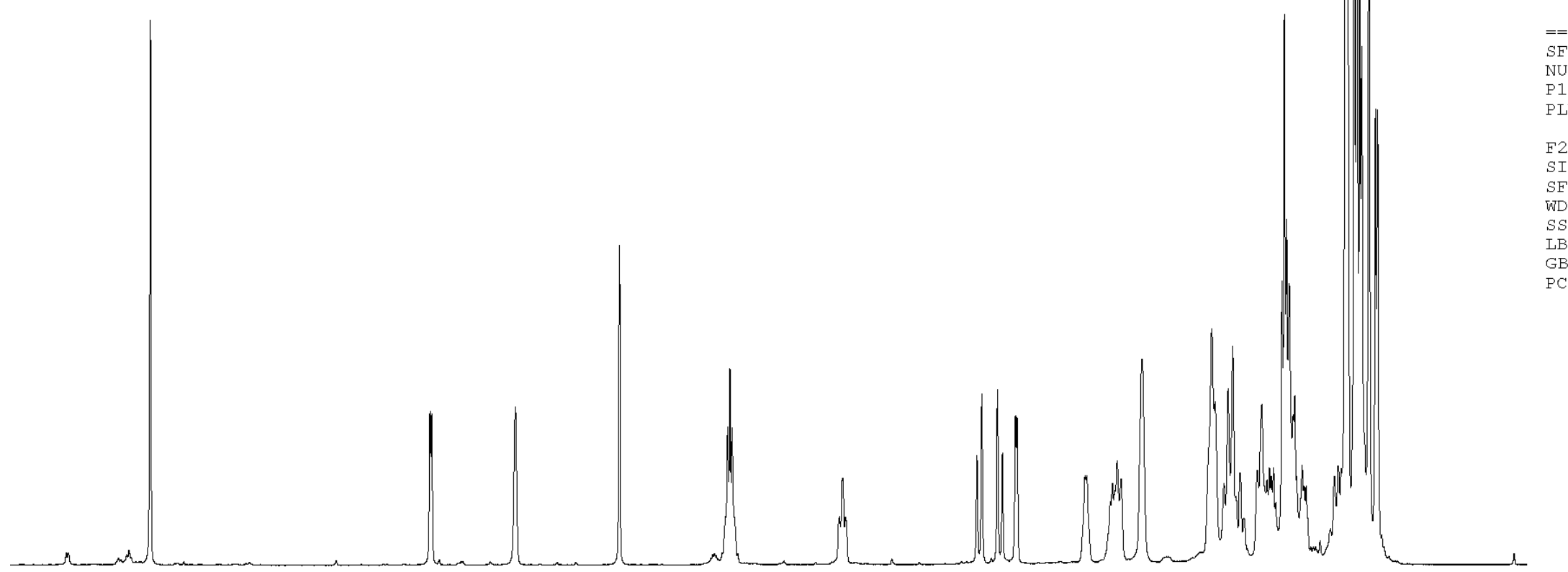

SFO

NUC1

1

$\mathrm{P}$

PLW1

600.1342009

F2 - Processing paramet SI $\quad 65536$ $\begin{array}{lr}\text { SF } & 600.1300345 \\ \text { WDW } & \end{array}$

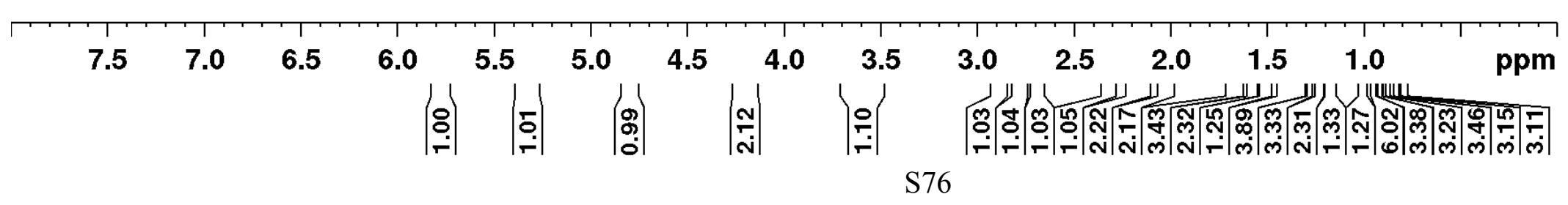




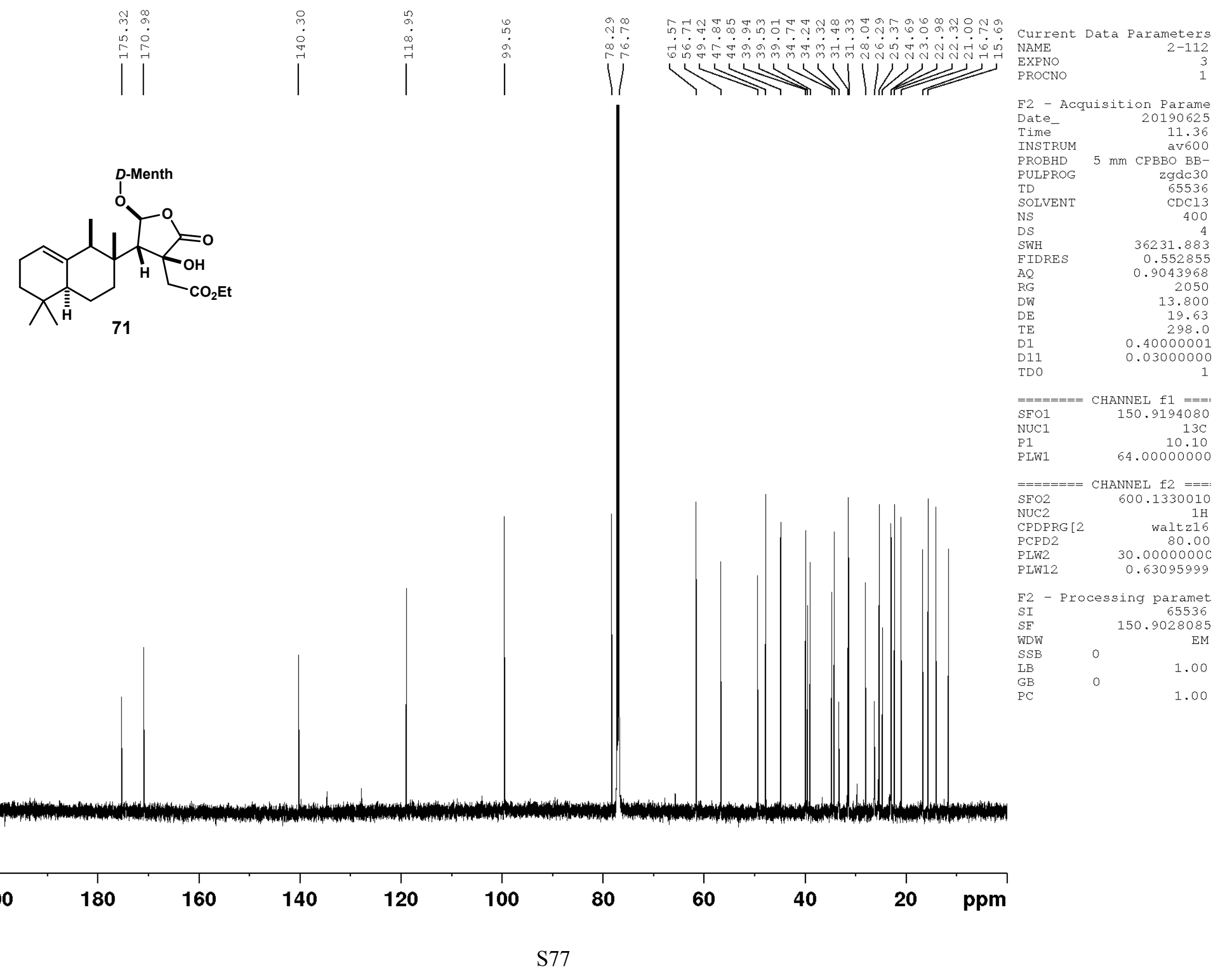




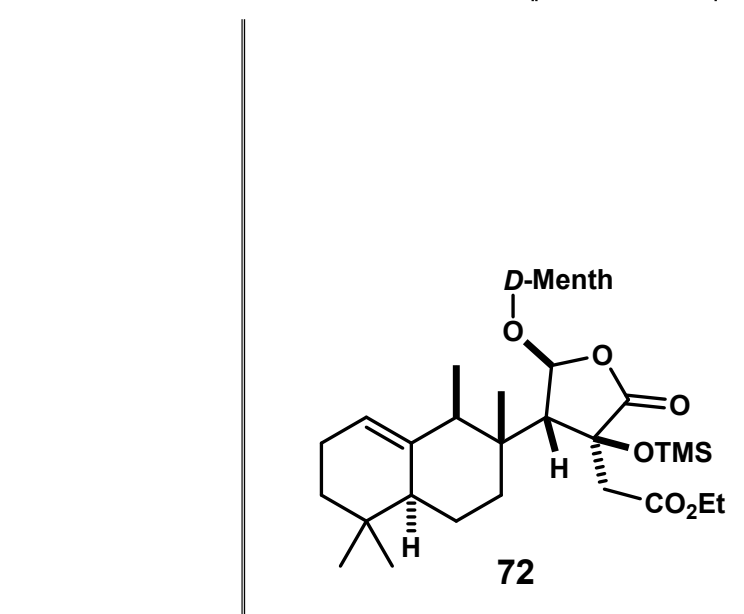

F2 - Acquisition Parame
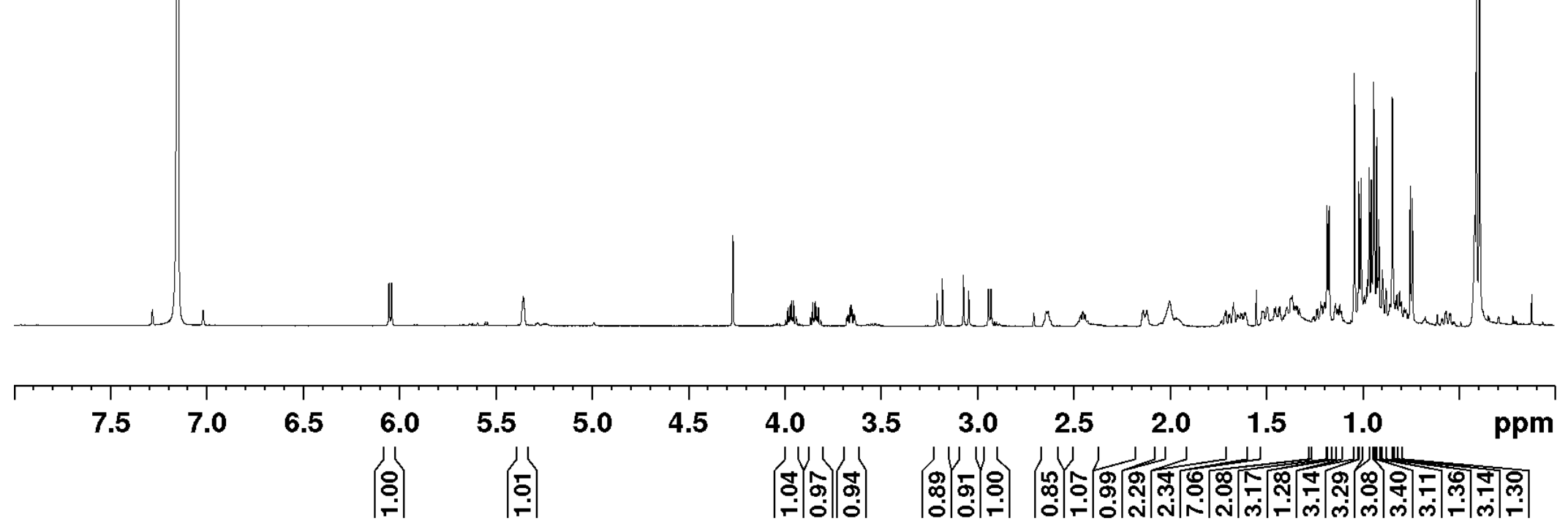


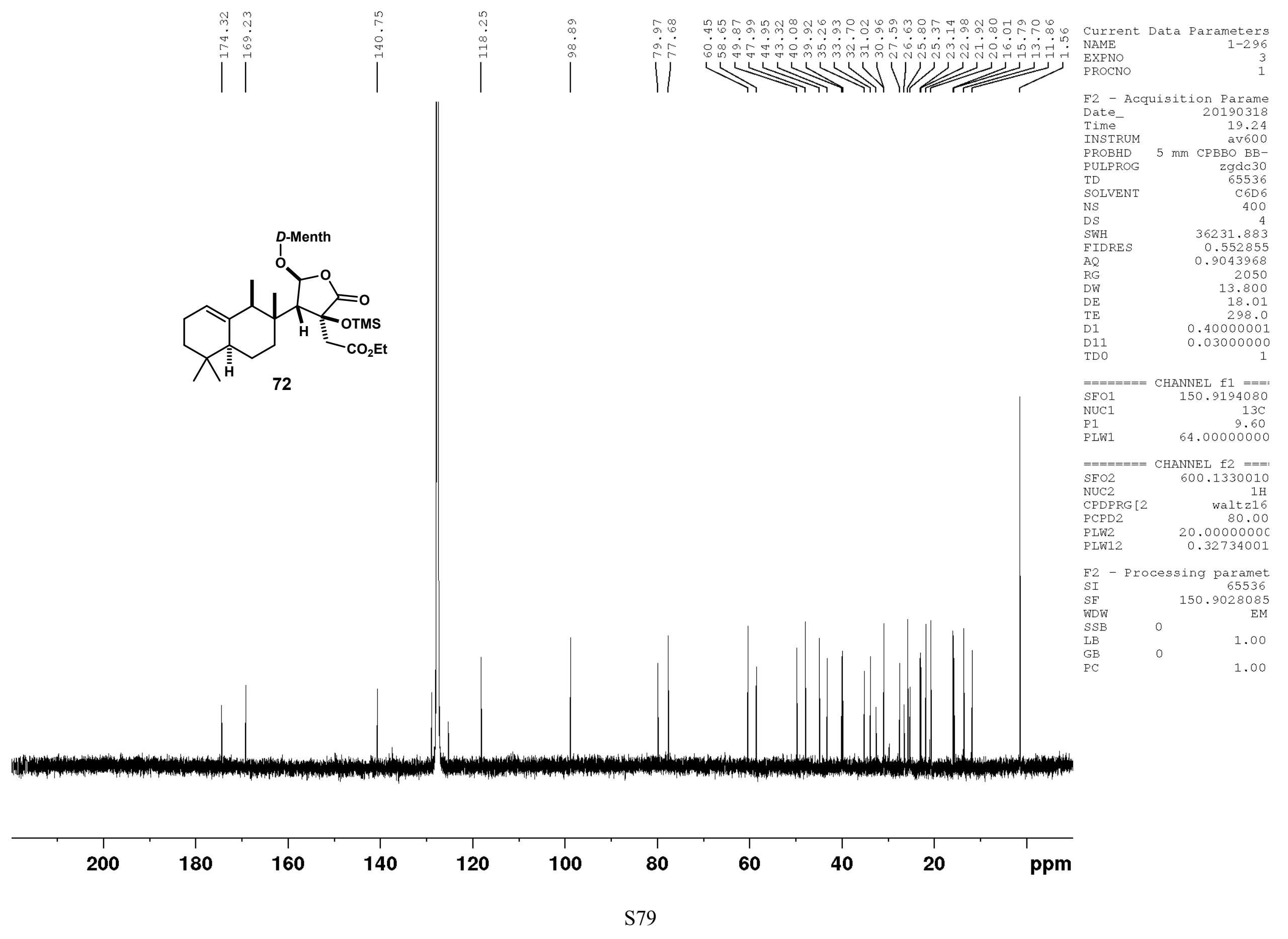



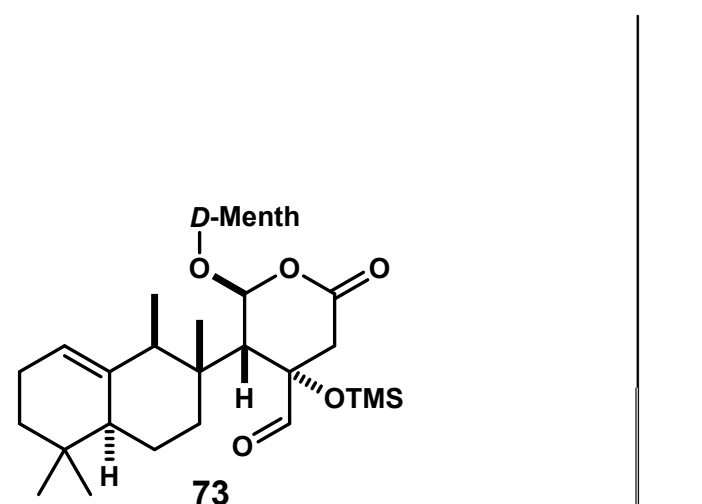

F2 - Acquisition Parame Date 20190321
10.08 TINTRUM - 5 m CPBBO PROBHD $5 \mathrm{~mm}$ CPBBO BBPROBHD zg30
98074 TD $\quad 98074$ NS DS SWH $\quad 9615.385$ FIDRES $\quad 0.098042$ $\begin{array}{lr}A Q & 5.0998478 \\ R G & 10\end{array}$ DW $\quad 52.000$ DE $\quad 13.81$ $\begin{array}{lr}\text { TE } & 298.0 \\ \text { D1 } & 0.10000000\end{array}$ TD

$========$ CHANNEL $f 1===$ $\begin{array}{lr}\text { SFO1 } & 600.1342009 \\ \text { NUC1 } & 1 \mathrm{H}\end{array}$ $\begin{array}{lr}\text { NuC1 } & 1 \mathrm{H} \\ \mathrm{P} 1 & 11.50\end{array}$

PLW1 $20.0000000 \mathrm{C}$

F2 - Processing paramet SI 65536 $\begin{array}{ll}\text { SF } & 600.1300000\end{array}$
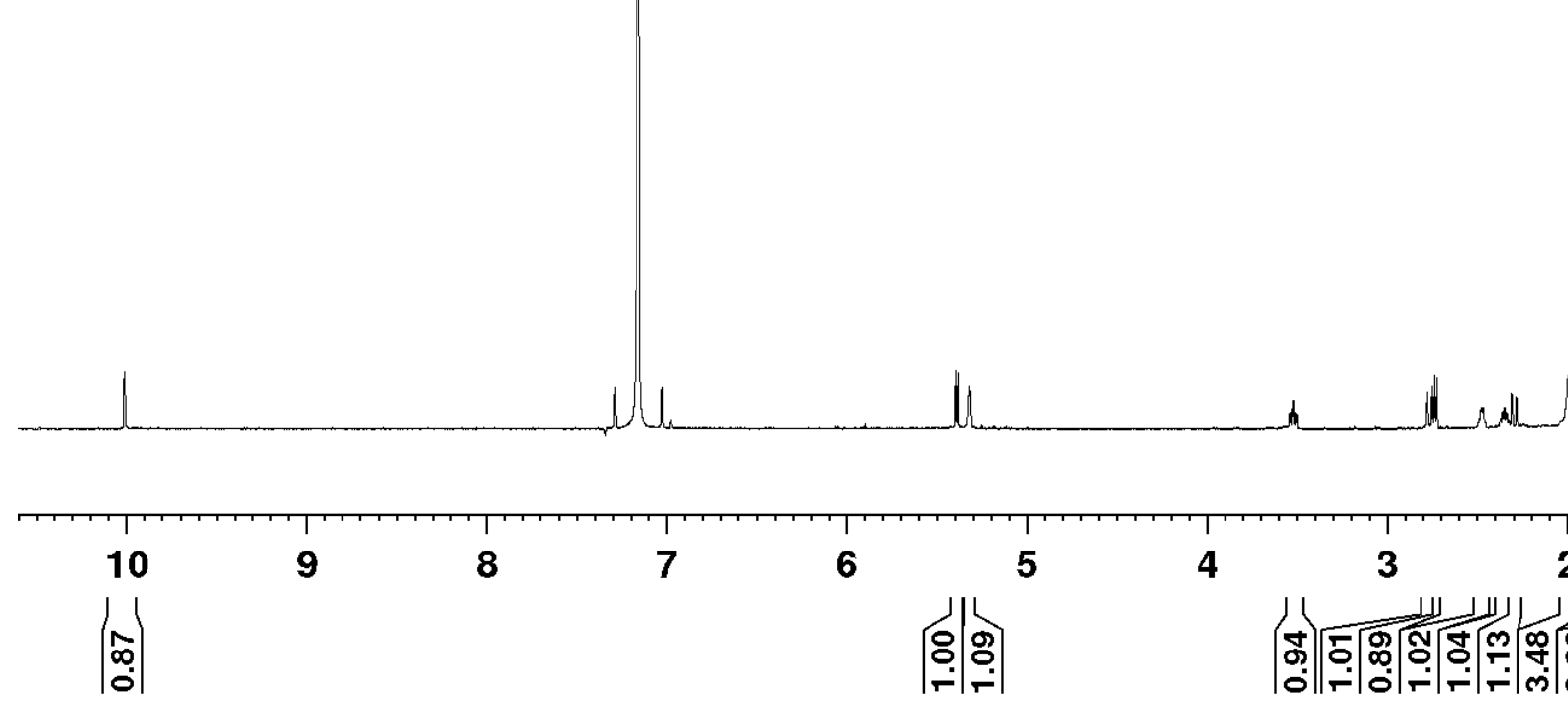

5

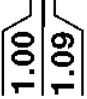

3

2

1

0

ppm

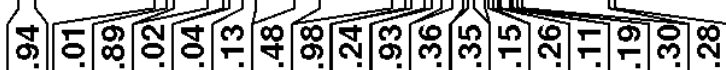

| 


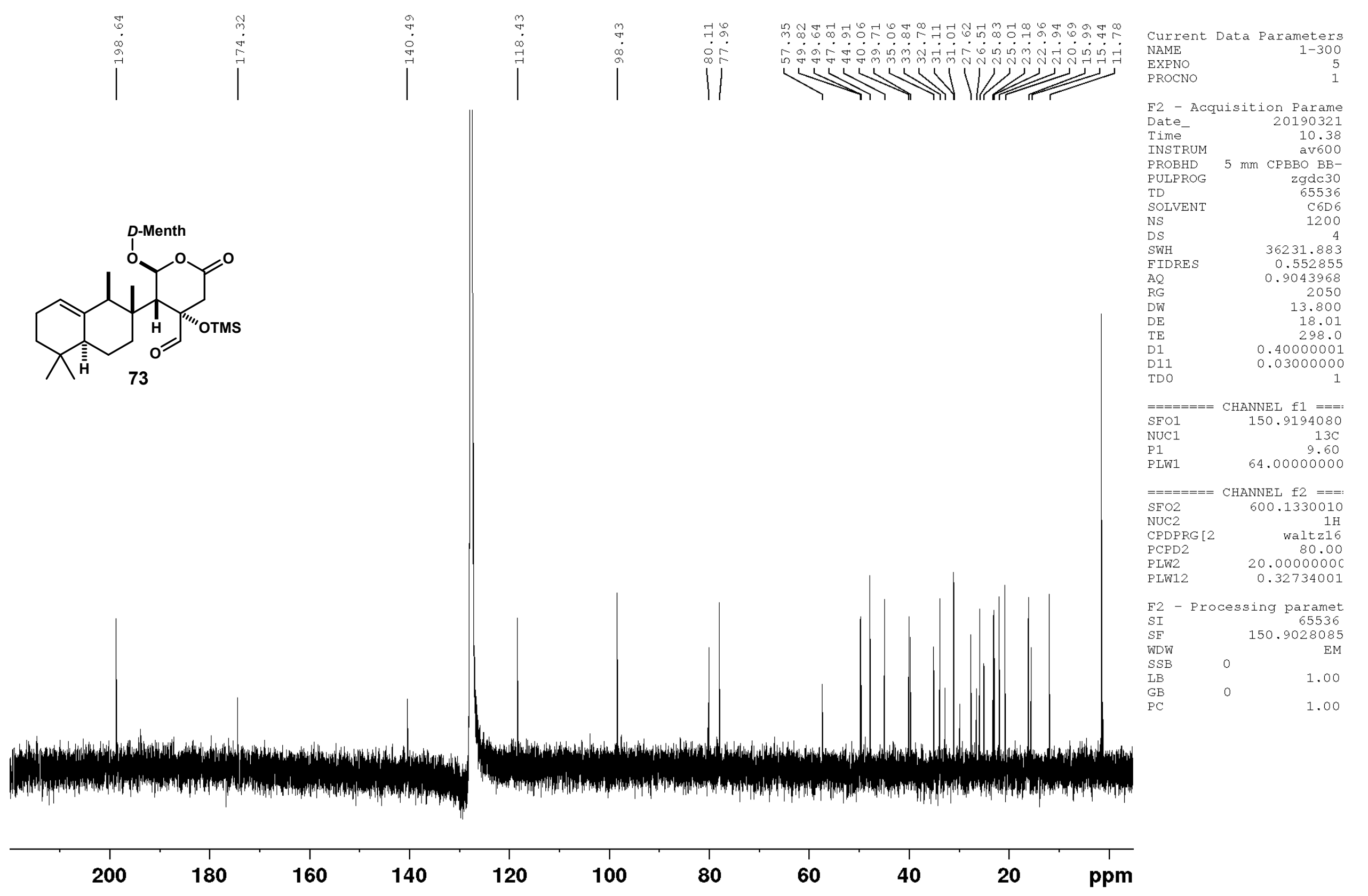




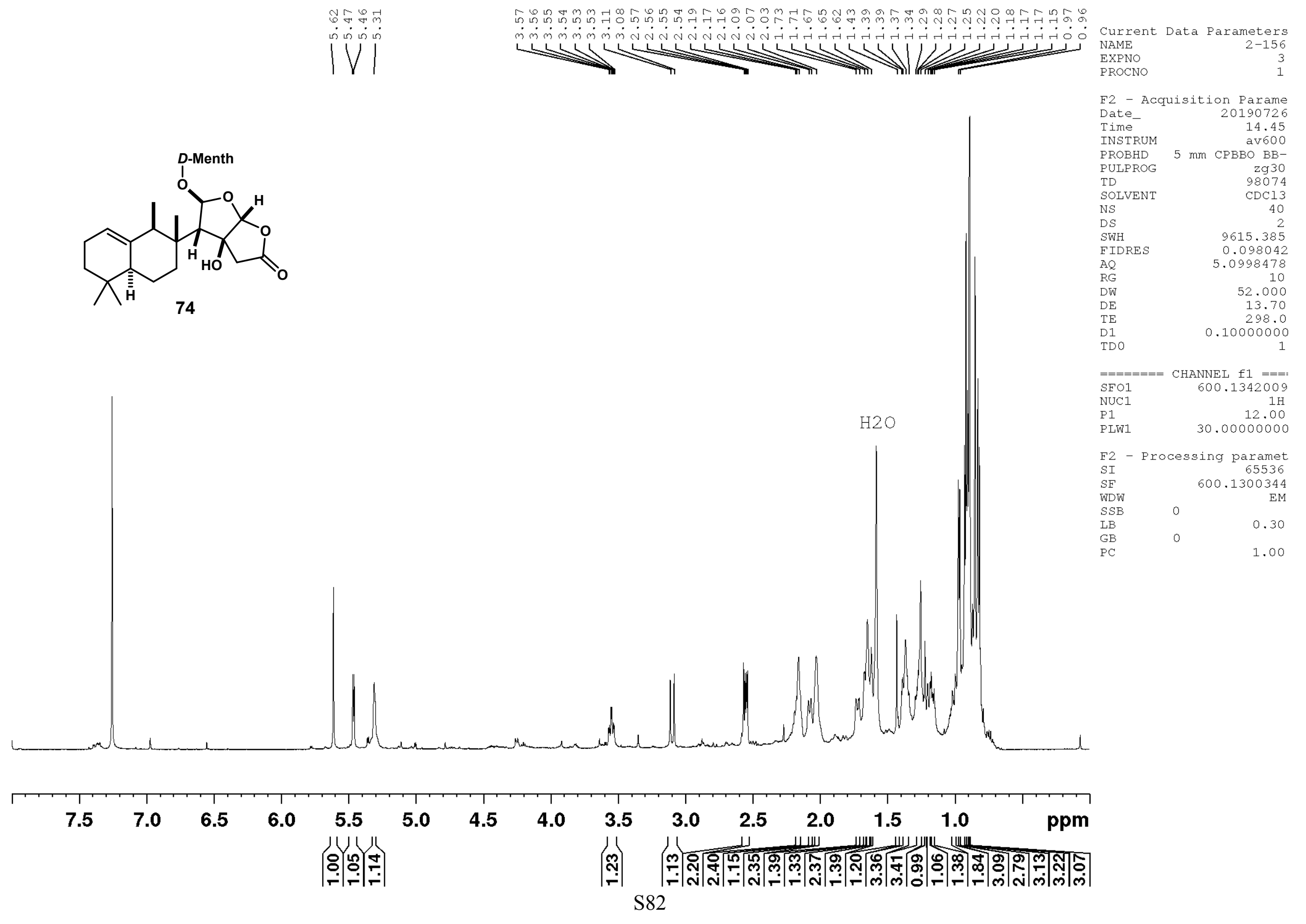




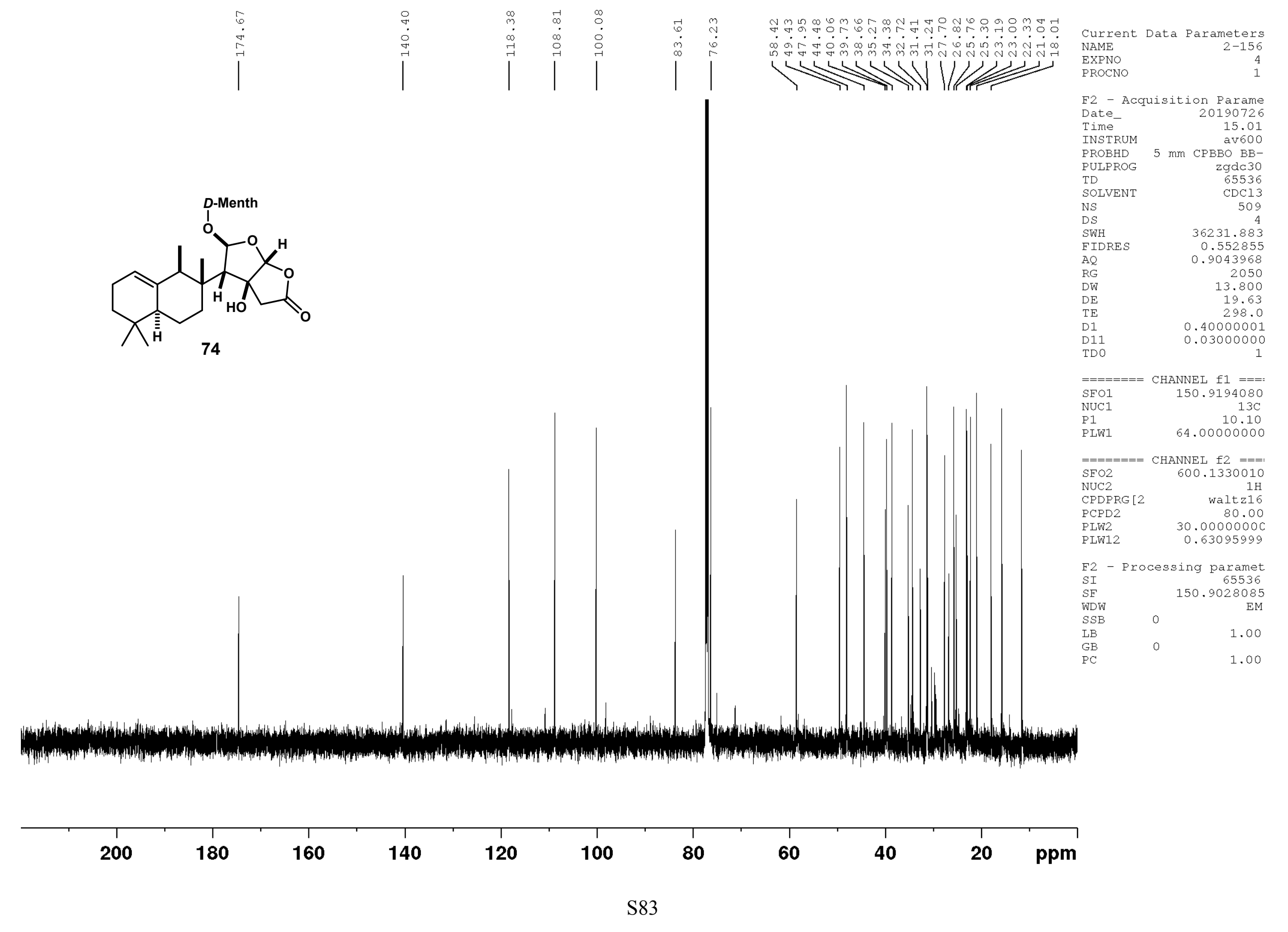




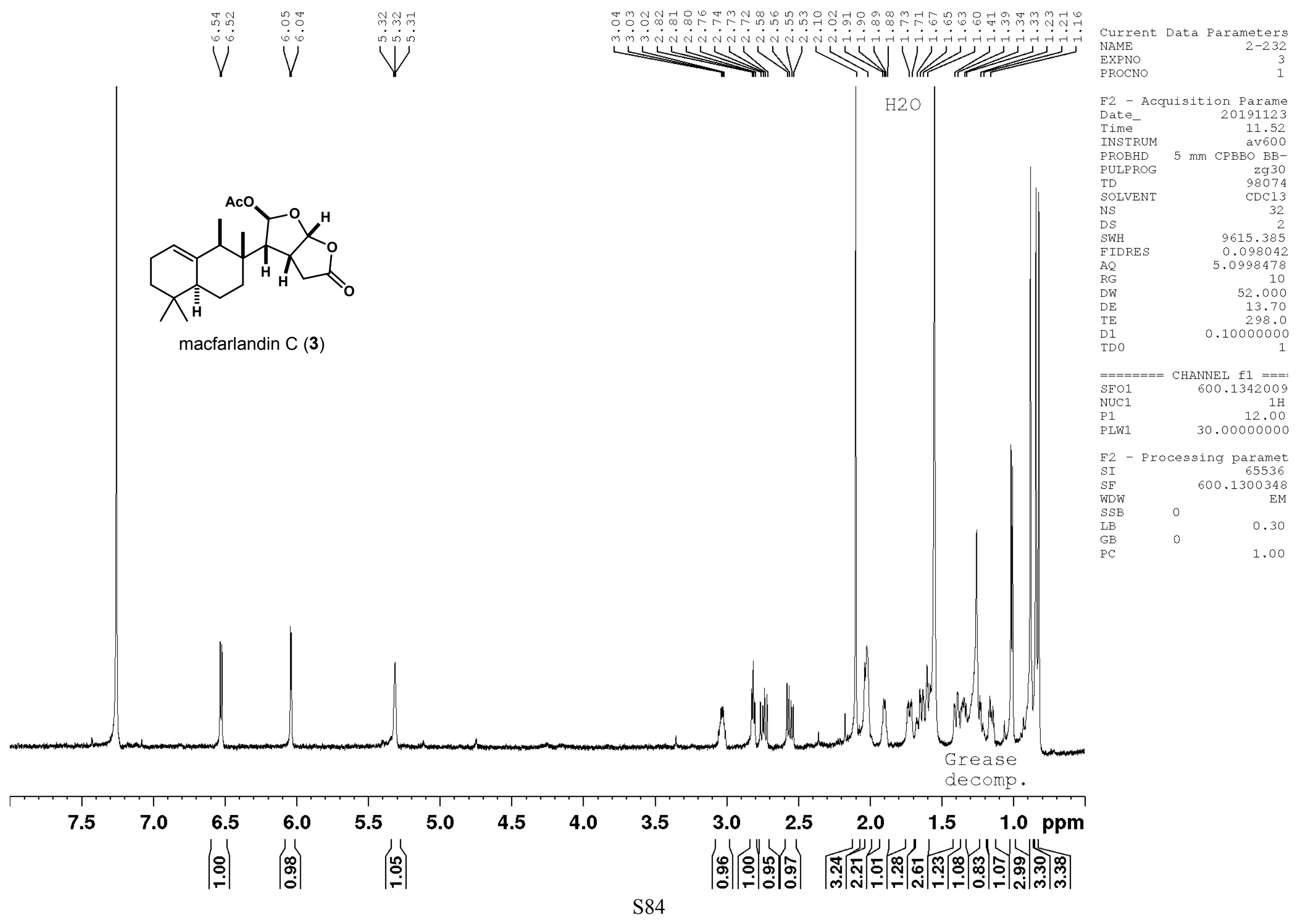




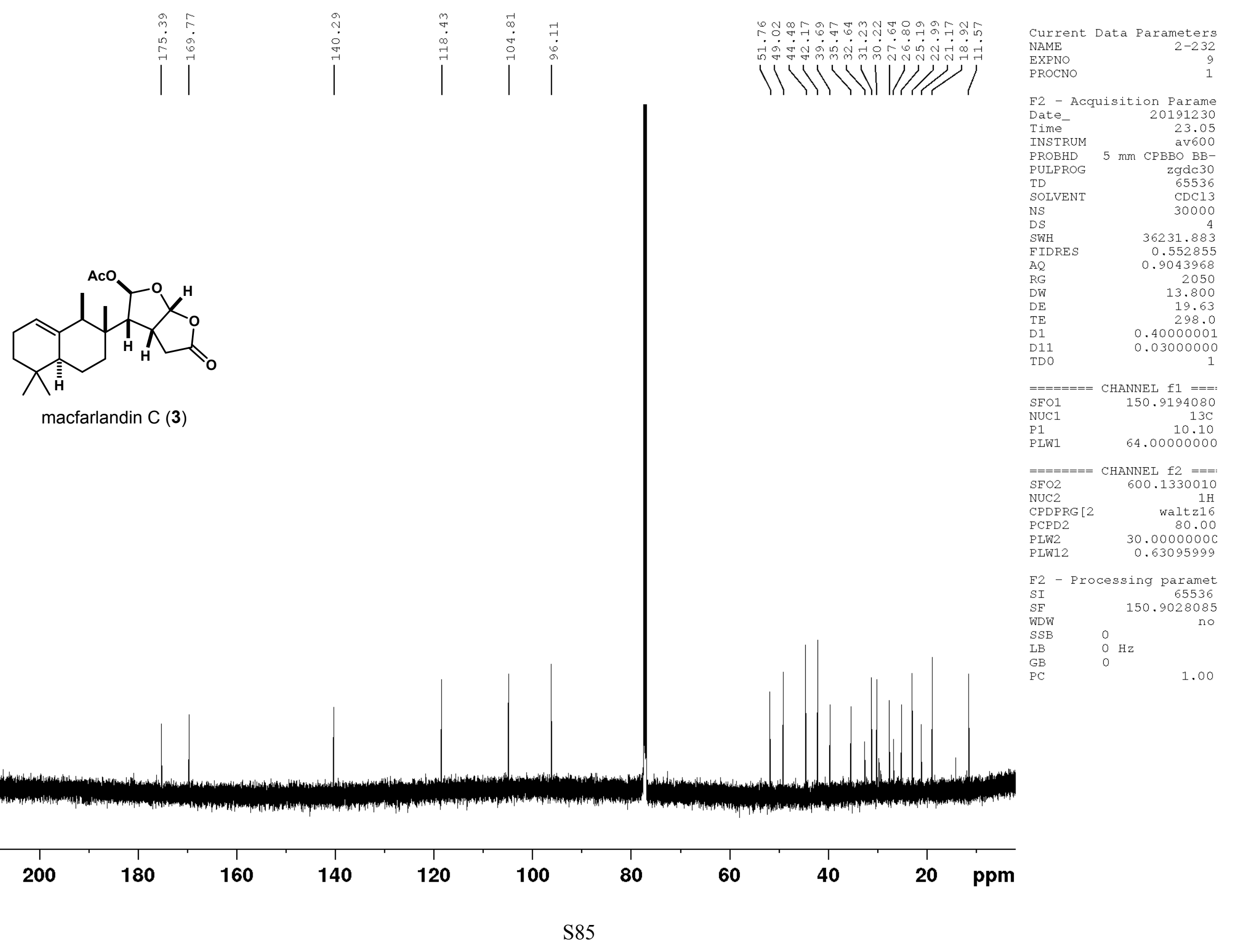




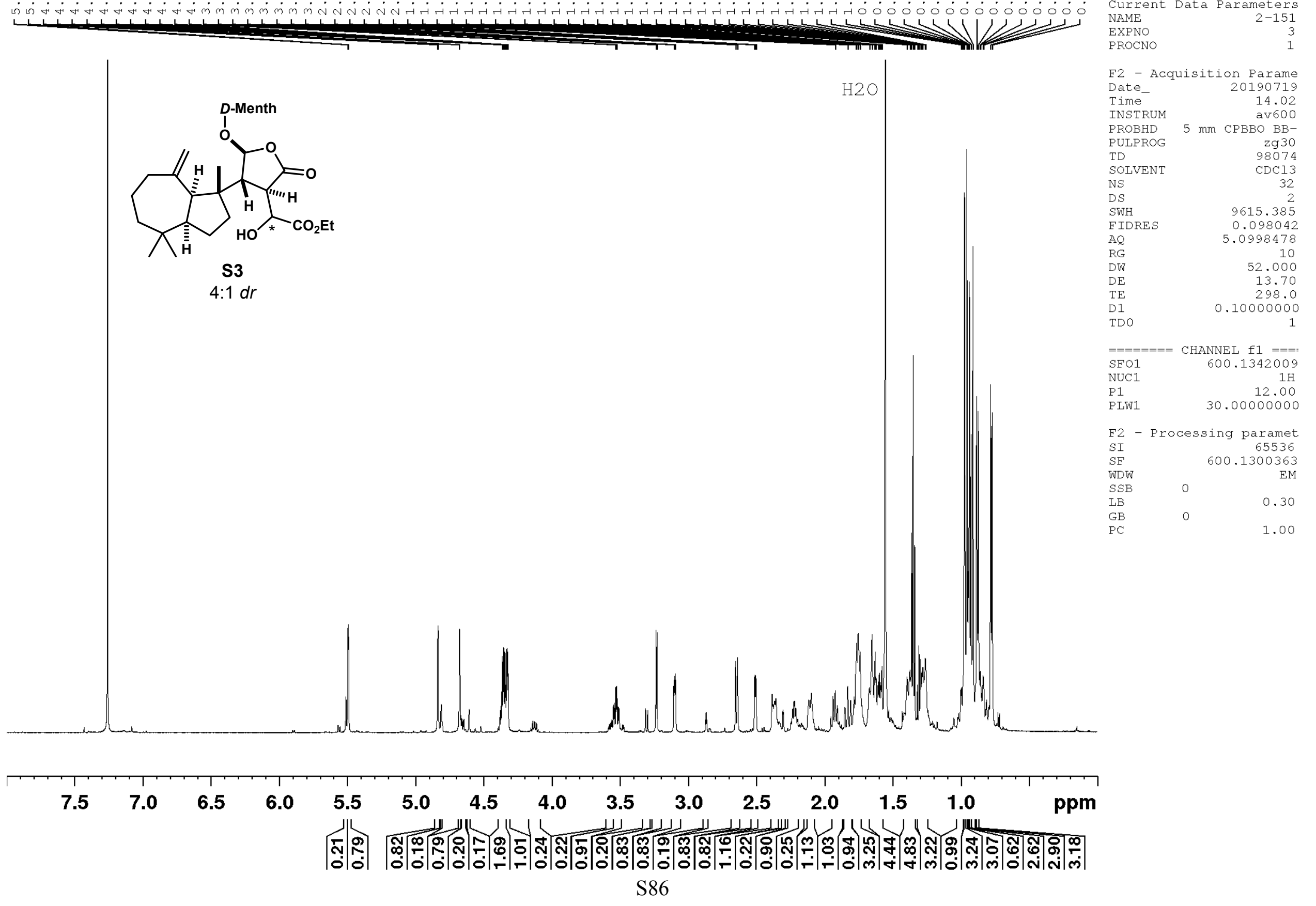




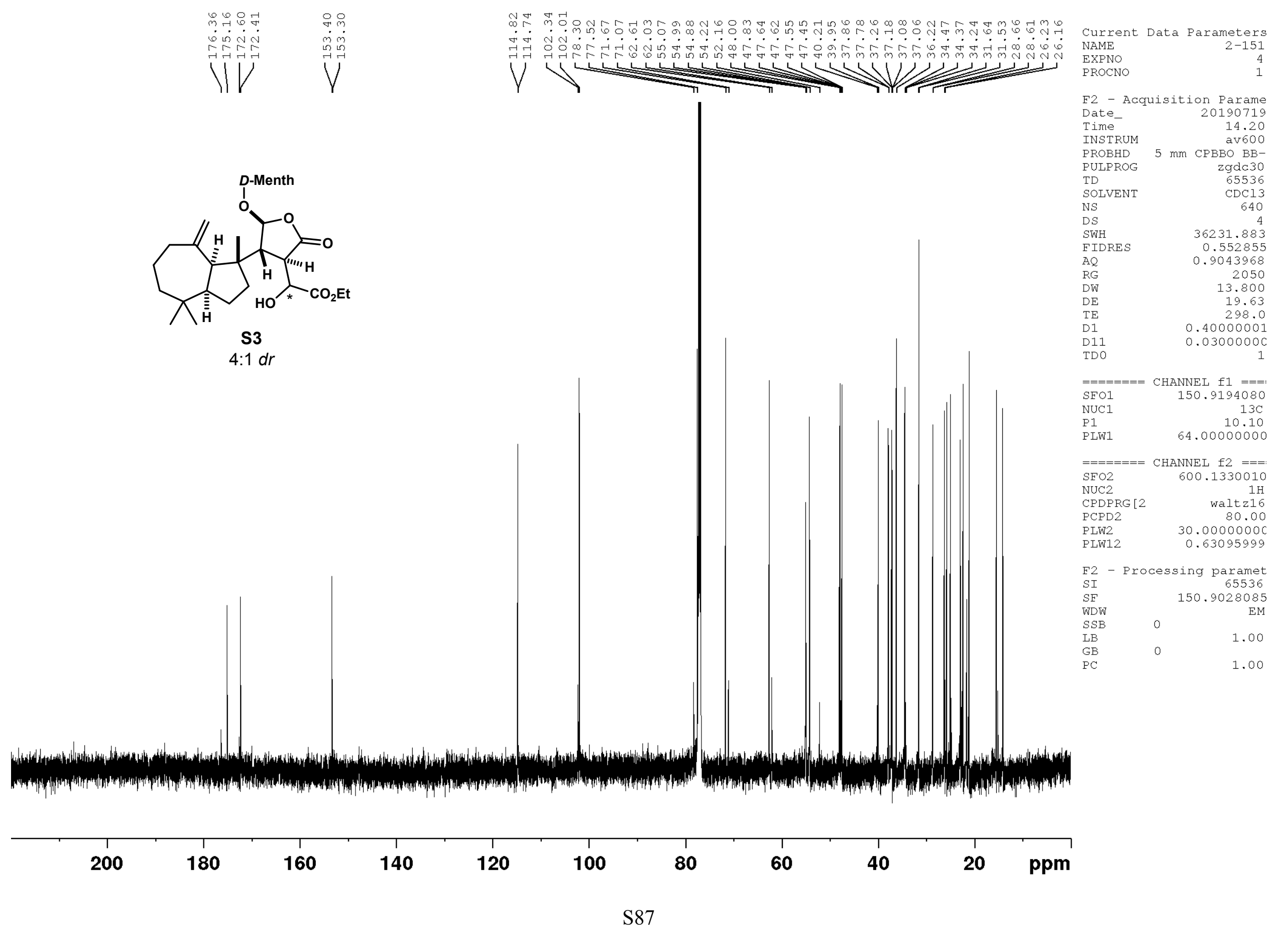




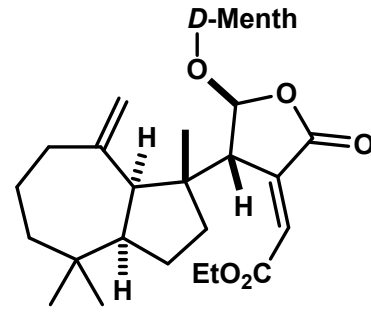

(E)-78

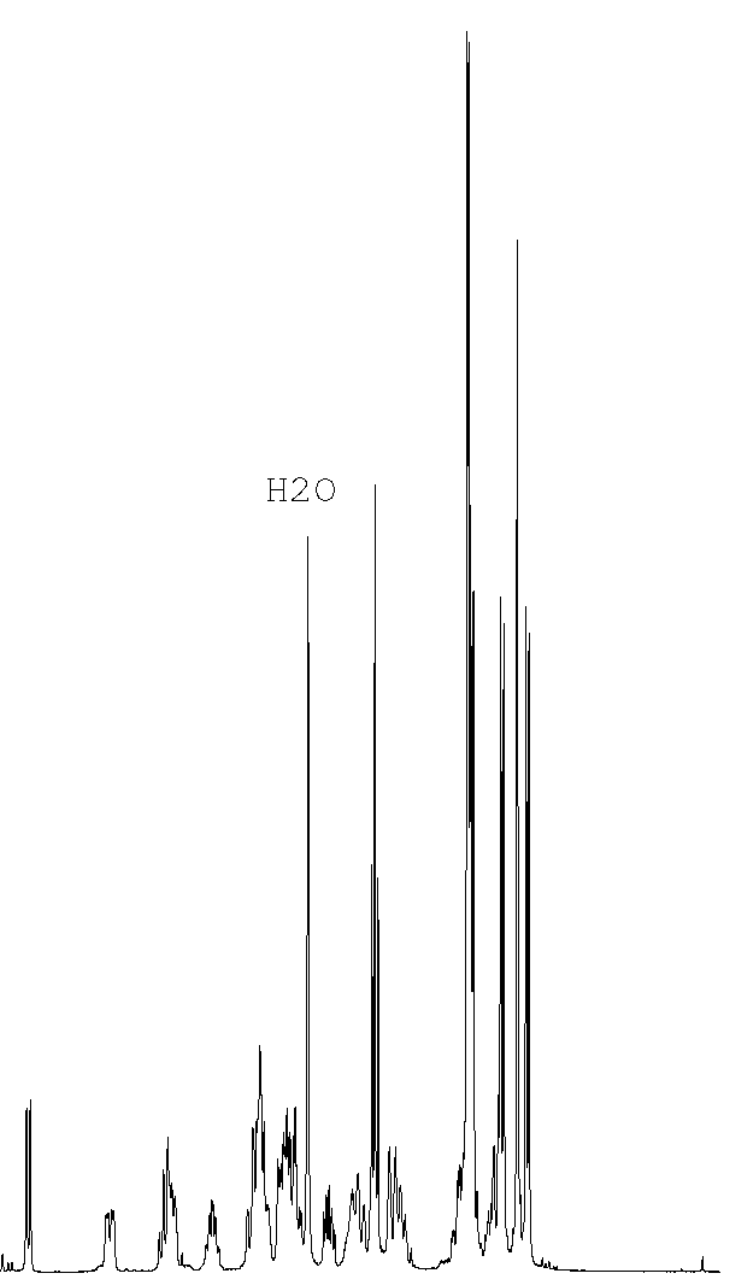

Current Data Parameters NAME

EXPNO

F2 - Acquisition Parame Date__ 20190722 Time

STRU

SOLVEN

NS

DS

SWH
FIDRES

$\mathrm{AQ}$

RG

DW

TE

TDO

SFO

SFO1

P1

PLW1

F2

$S I$

SF
WDW

SSB

$\mathrm{LB}$
$\mathrm{GB}$

$\mathrm{PC}$

$$
\begin{array}{r}
\mathrm{zg} 30 \\
98074 \\
\mathrm{CDCl} \\
32 \\
2 \\
9615.385 \\
0.098042 \\
5.0998478 \\
10 \\
52.000 \\
13.70 \\
298.0 \\
0.10000000
\end{array}
$$

1

CHANNEL $\mathrm{f} 1===$

$1 \mathrm{H}$
12.00

30.00000000

Processing paramet 600.1300363

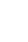

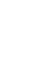

0.30

1.00

\section{$7.5 \quad 7.0$ 7.0

$6.5 \quad 6.0$

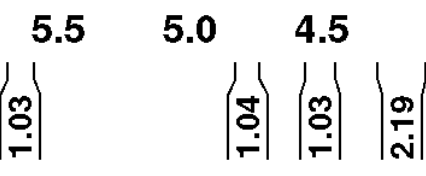 \\ $4.0 \quad 3.5$ \\ 3.0 \\ 2.52 .0 \\ $1.5 \quad 1.0$ \\ ppm \\ $\mid$ \\ |

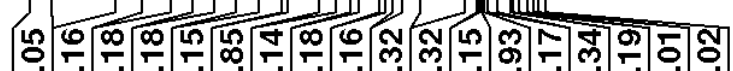

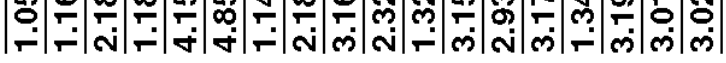




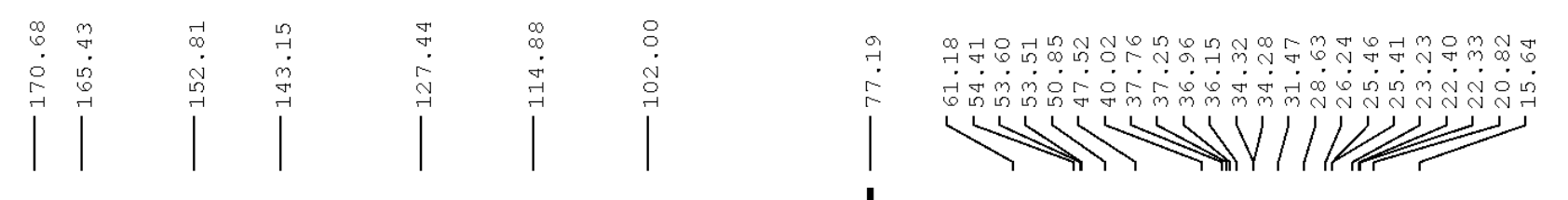

Current Data Parameters

NAME

EXPNO
PROCNO

F2 - Acquisition Parame

Date_

Iime

20190722

15.29
av 600

$5 \mathrm{~mm} \mathrm{CPBBO} \mathrm{BB}-$

zgda 30

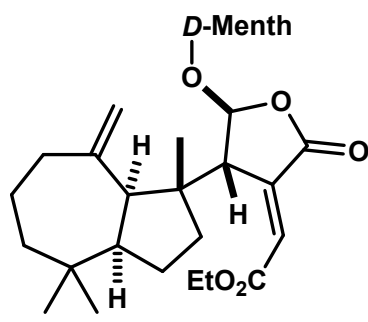

(E)-78

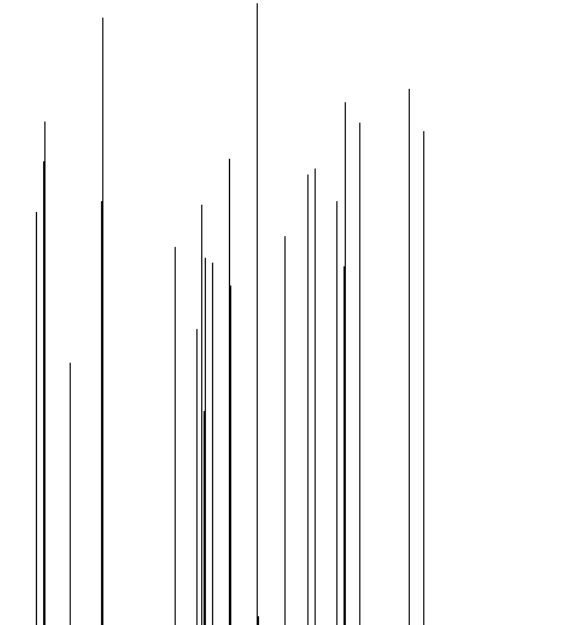

SOLVENT

NS

DS

RG

DW

TE

D1
D11

TDO

SFOI

SFO1
NUC1

295536
6553

PLW1

640
4
36231.883
0.552855 0.552855
0.9043968

2050
13.800

19.63

0.40000001

0.03000000

CHANNEL $\mathrm{f} 1===$

150.9194080
$13 \mathrm{C}$

64.00000000

SFO2 $====$ CHANNEL $\mathrm{f} 2===$

$\mathrm{SFO} 2$ 600.1330010
$1 \mathrm{H}$

CPDPRG [2 waltz16

PLW2 30.00000000

PLW12

F2 - Processing paramet

SI 65536

$\begin{array}{lr}\text { SF } & 150.9028085 \\ \text { WDD } & \text { FM }\end{array}$

WDW

LB

$G B$

1.00

1.00 


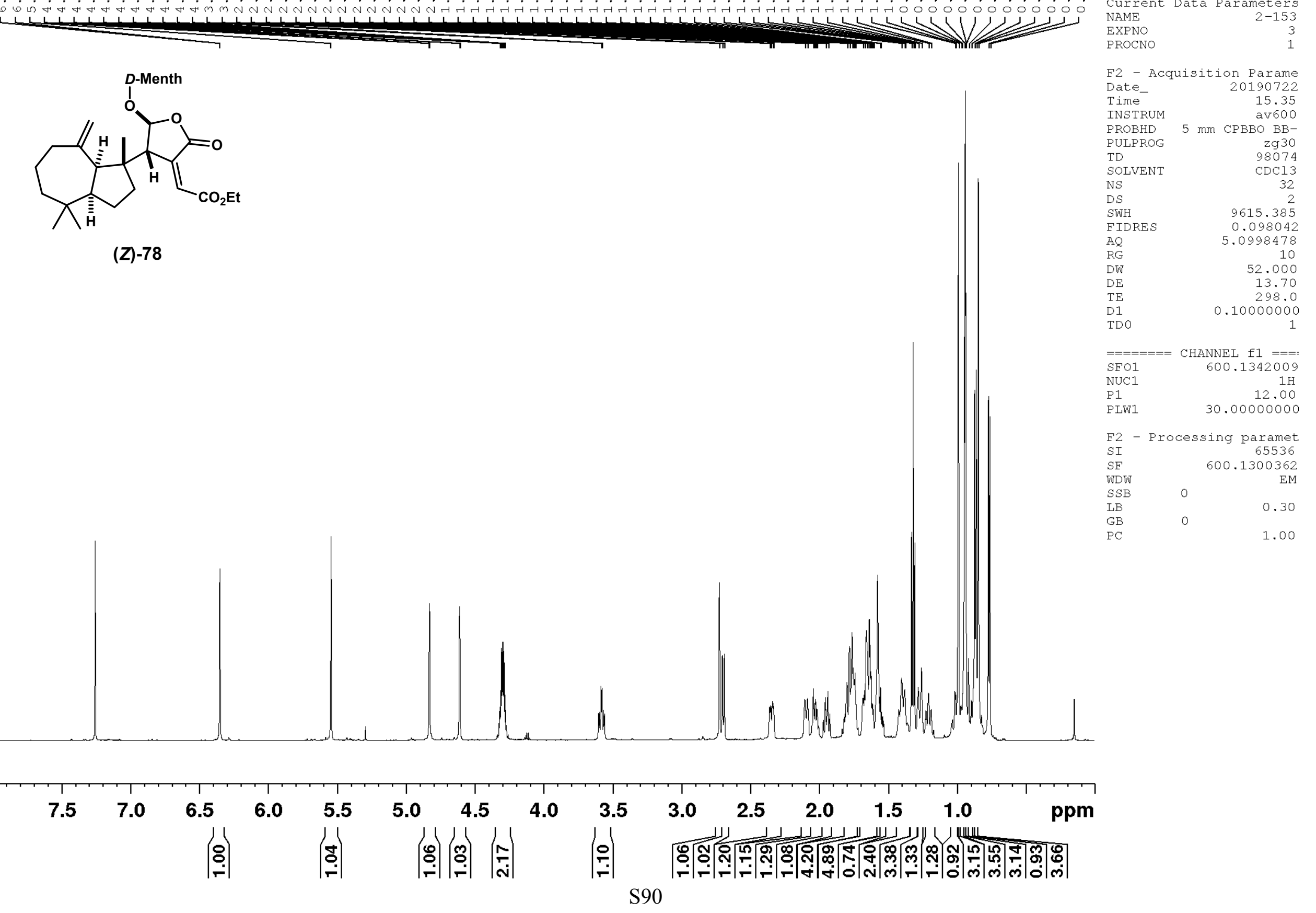




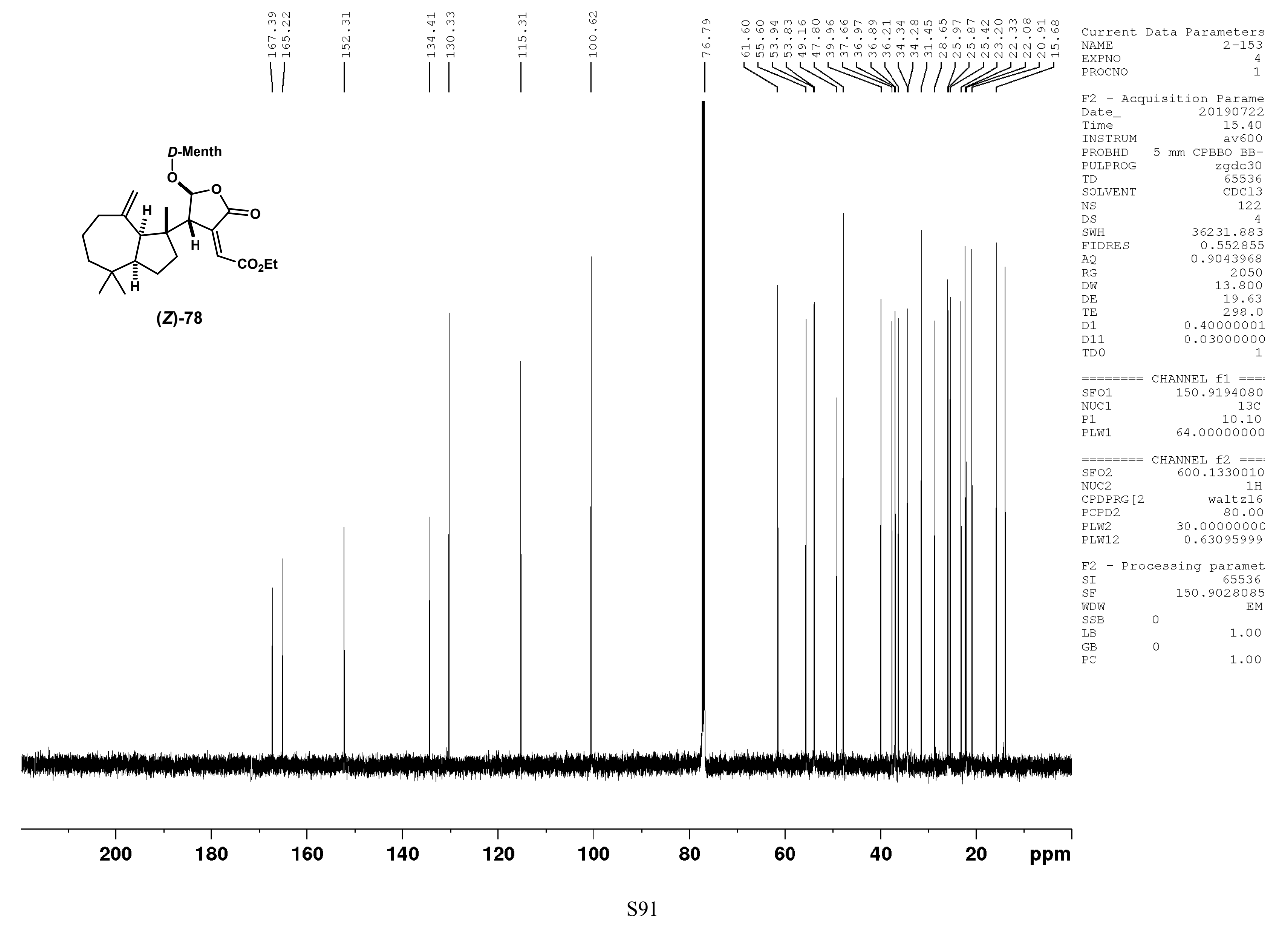




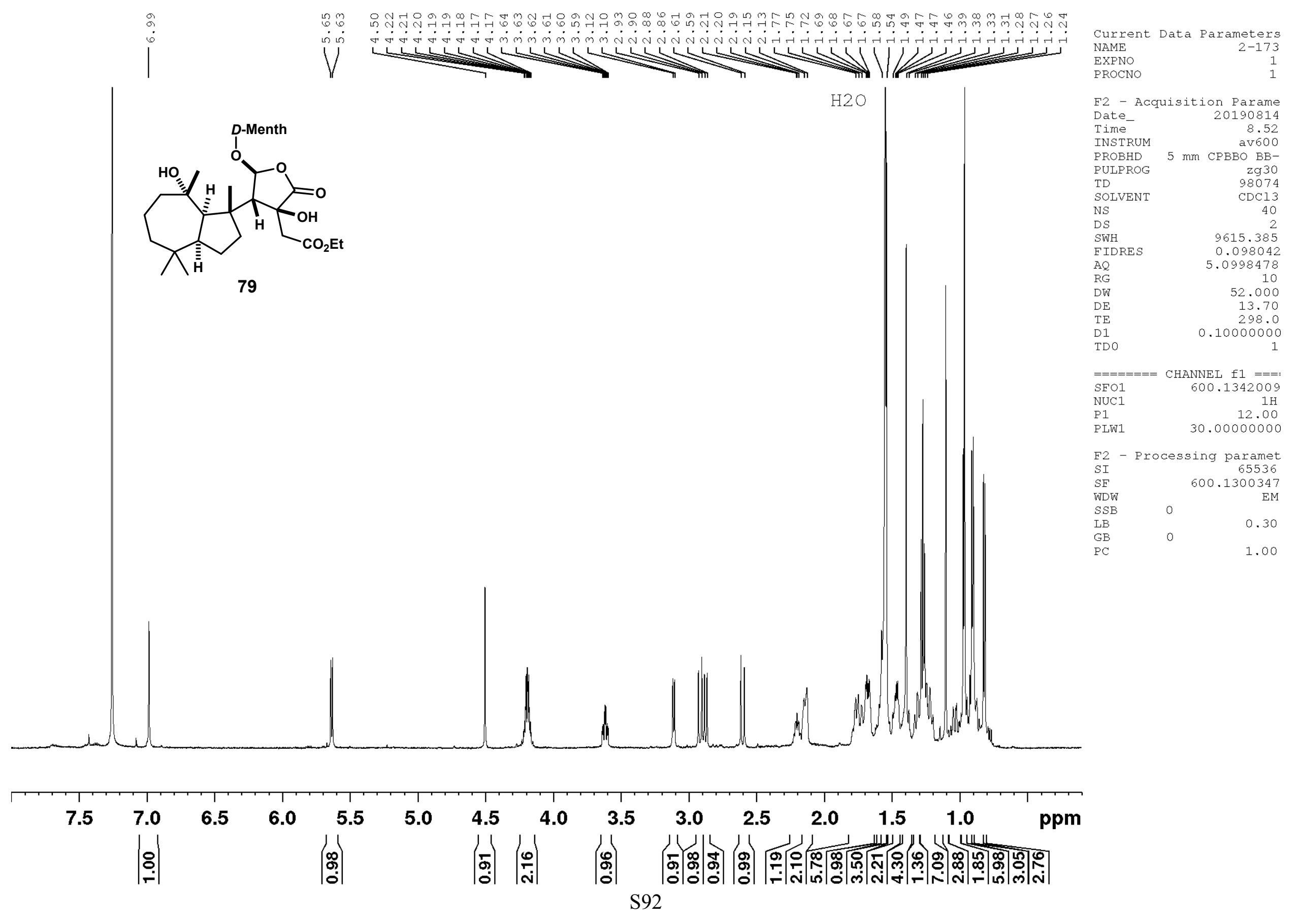




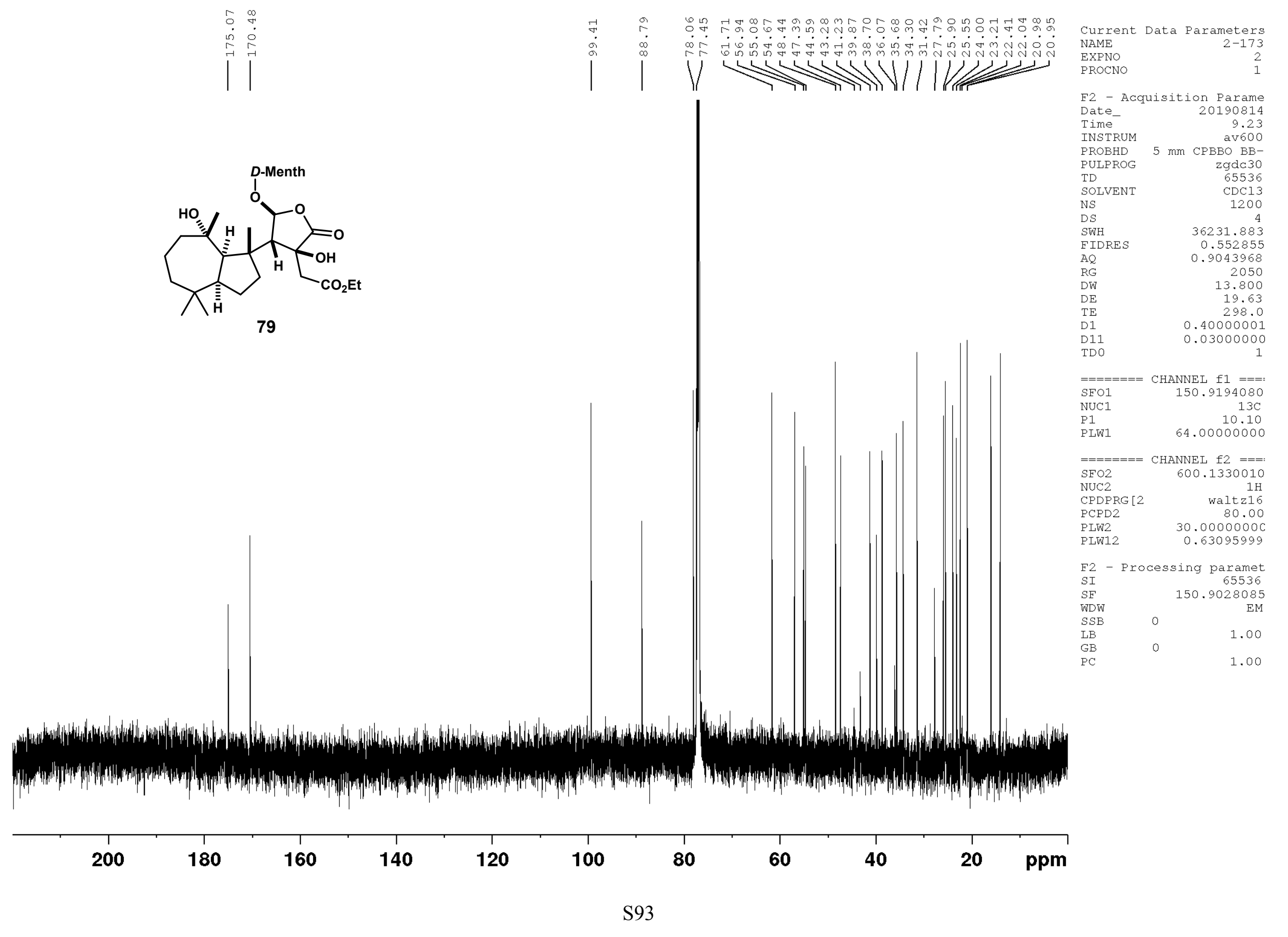




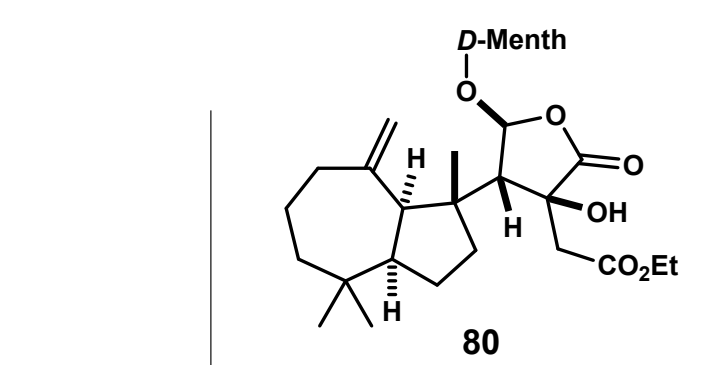

F2 - Acquisition Parame

Processing paramet 65536
600.1300351
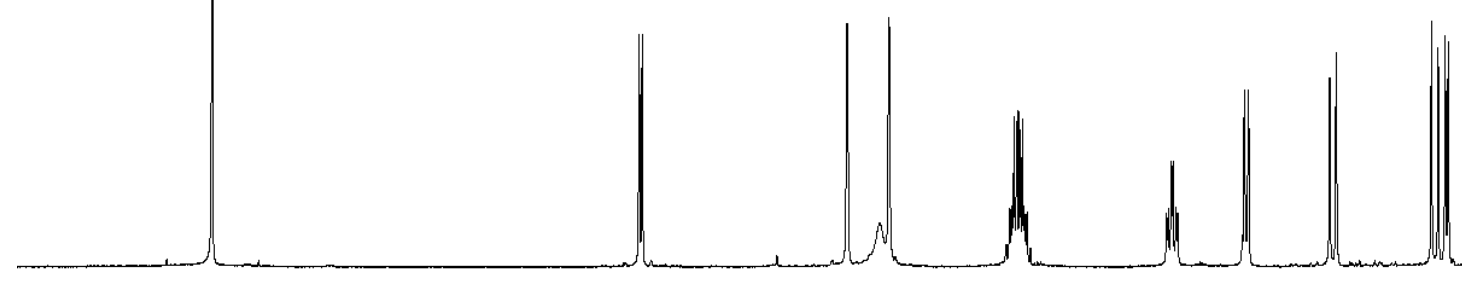

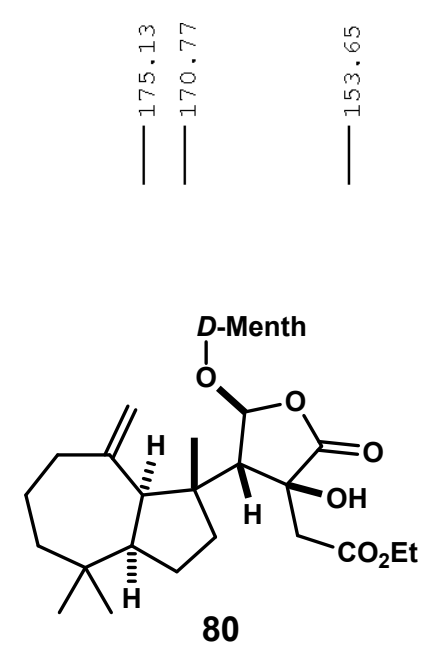

Im. I.
180

160

140

100

80

60

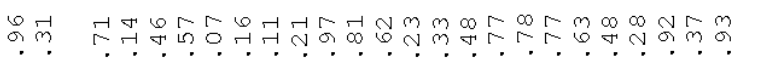
당

V

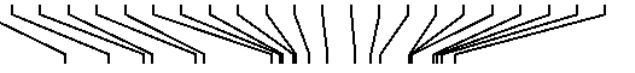

NAME

EXPNO
PROCNO

ameters

F2 - Acquisition Parame

Date

Time

PROBHD

PULPROG

TD
SOLVENT

NS

DS

SWH
FIDRES

$A Q$

RG

$\mathrm{DW}$

TE

D1

D11
TD0

$=====$
SFO1
NUC1

P1
PLW1

20190808

19.43
av600

$\mathrm{mm}$ СPBBO $\mathrm{BB}-$

zgde 30

65536

160
4

36231.883

0.9043968

2050

13.800
19.63

298.0

0.40000001

0.03000000

$=$ CHANNEL $f 1===$

$=======$ CHANNEL $\mathrm{f} 2===$ :

$\mathrm{SFO} 2$

CPDPRG [2

PCPD2

PLW12

600.1330010
$1 \mathrm{H}$
waltz16
80.00

$30.0000000 \mathrm{C}$

F2 - Processing paramet

$\begin{array}{lr}\text { SI } & 150.9028085 \\ \text { SF } & \end{array}$

WDW EM

1.00

$\mathrm{PC}$

1.00 
IAME EXPNO

F2 - Acquisition Parame

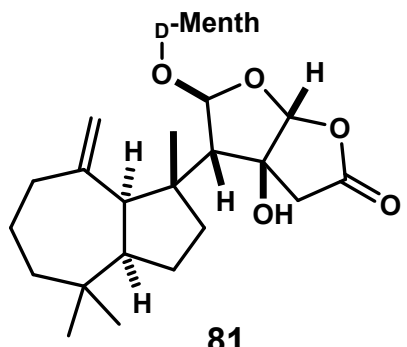

Date

Time

INSTRUM

PROBHD

PULPROG

TD

SOLVENT

NS

SWH

FIDRES

AQ

RG

81

$\mathrm{DW}$
$\mathrm{DE}$
$\mathrm{TE}$

TE

TDO

20190802

10.19

mm $\mathrm{CPBBO} \mathrm{BB}-$

$\mathrm{zg} 30$

CDC13

40
2

9615.385

0.098042

.0998478
10

52.000

298.0

1

$==$ CHANNEL $\mathrm{f} 1===$

NUC1 $\quad 1 \mathrm{H}$

P1 12.00

PLW1 30.00000000

F2 - Processing paramet

SI 65536

$\begin{array}{ll}S F & 600.1300347\end{array}$
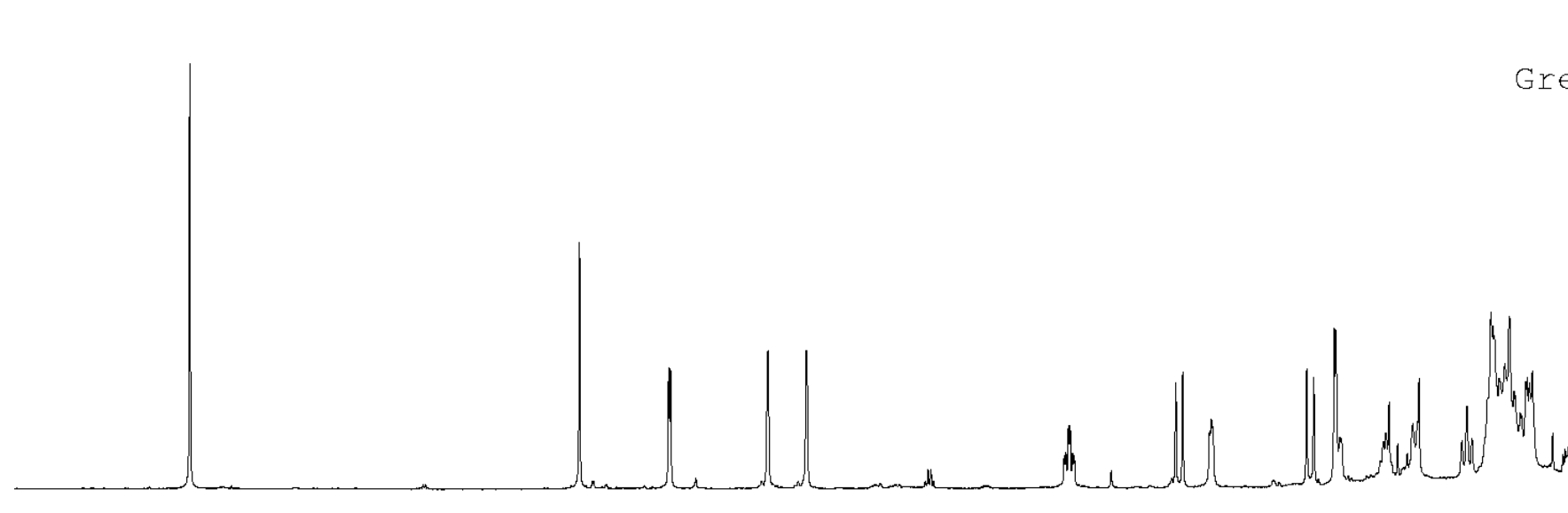$$
\text { (n) }
$$$$
0.30
$$

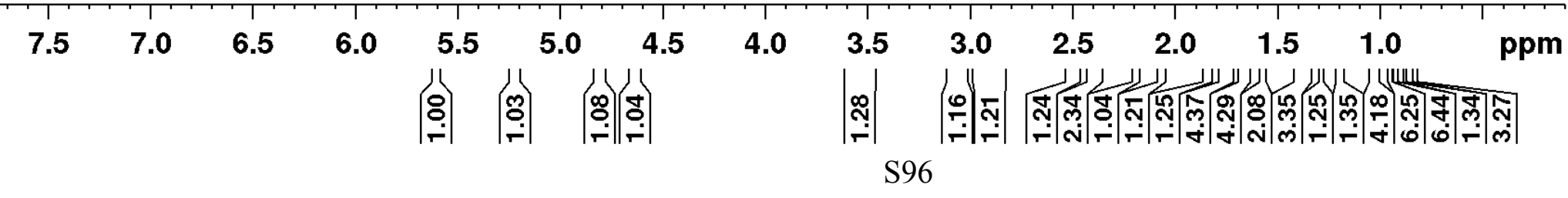




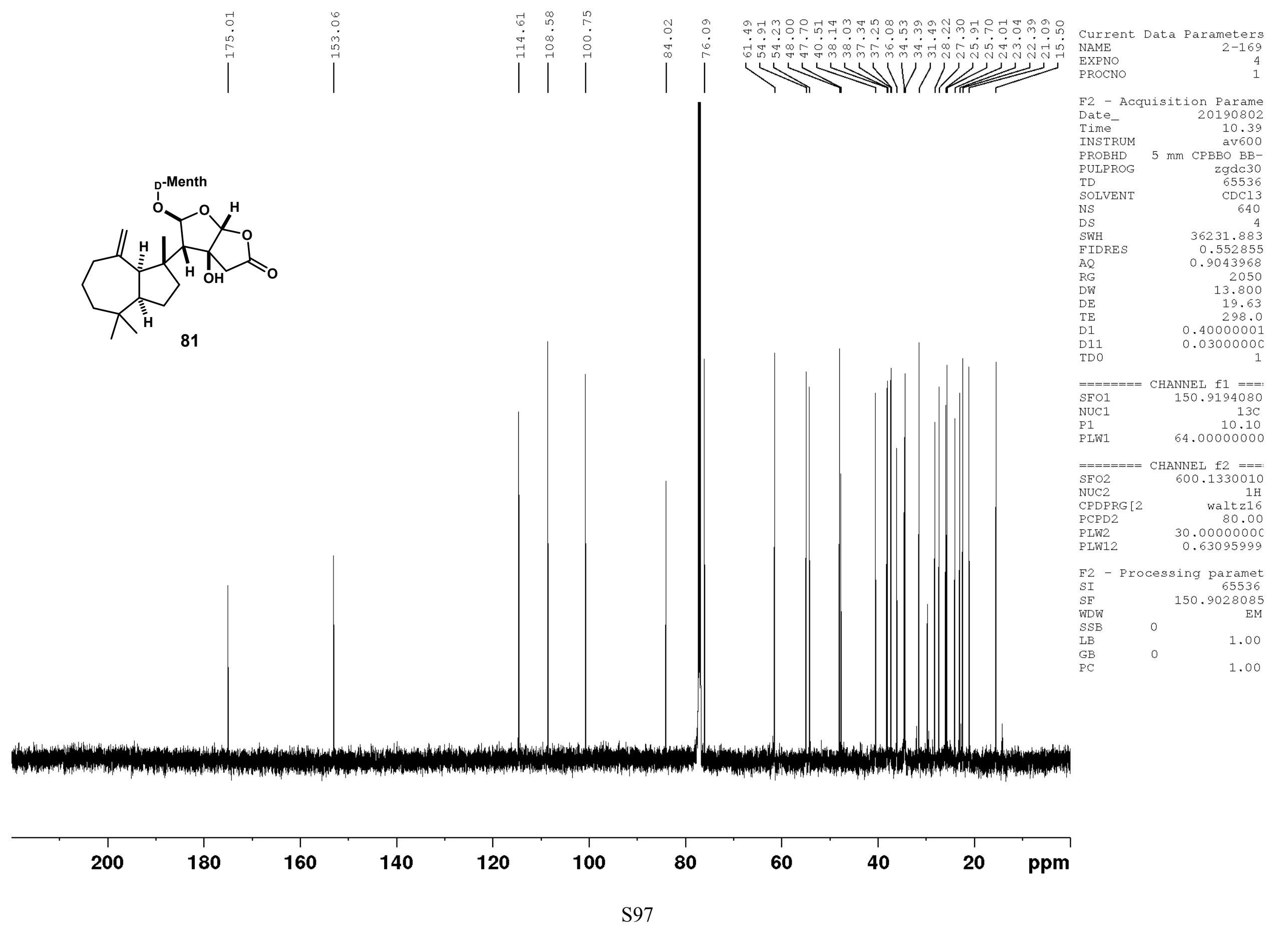




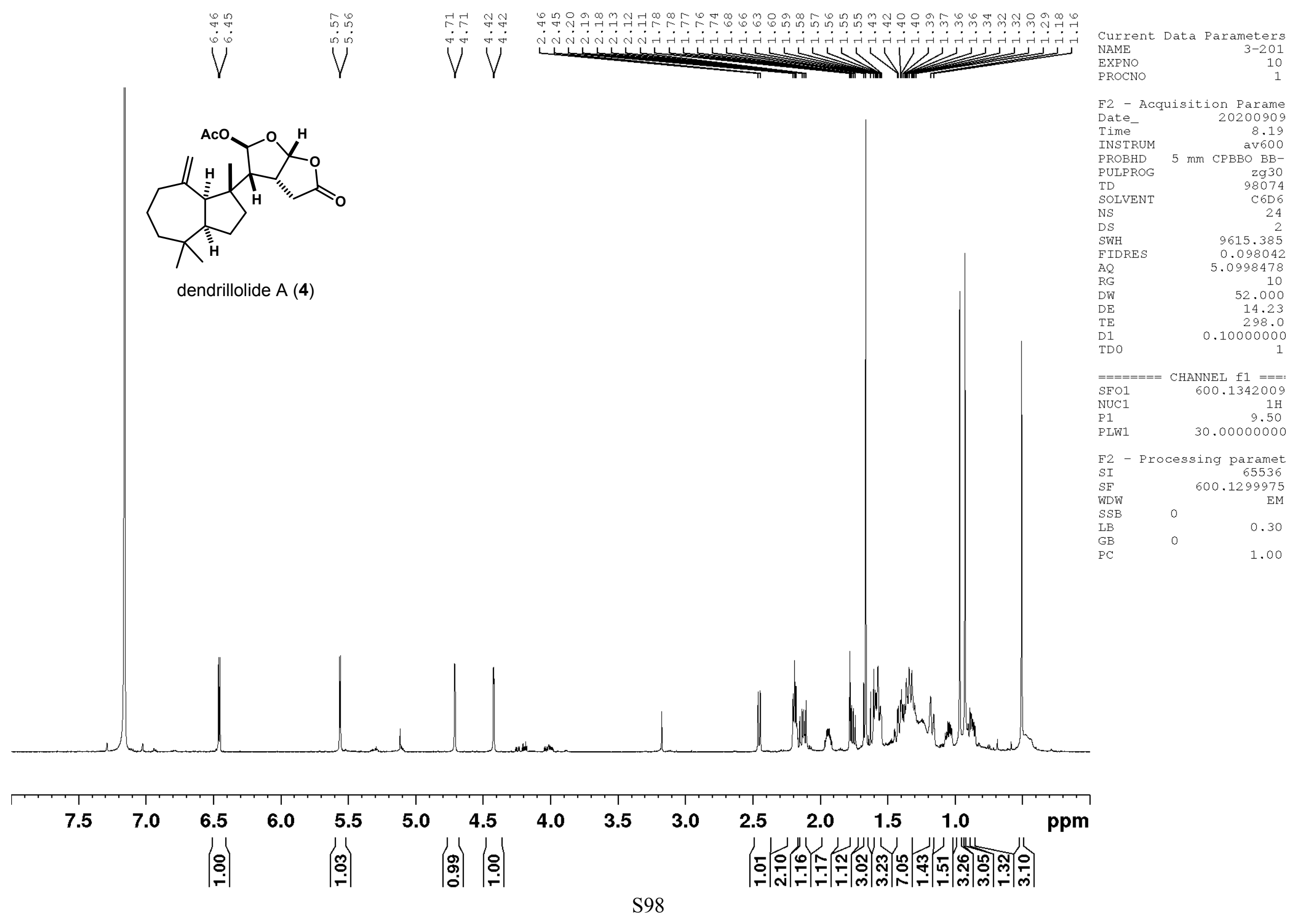



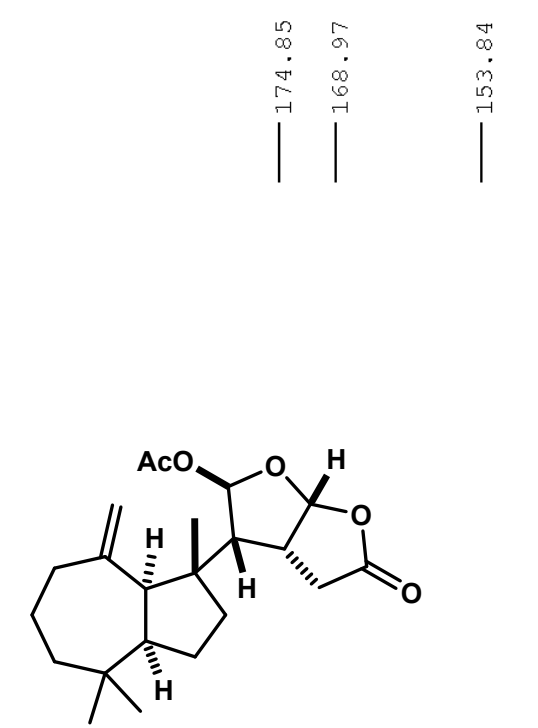

dendrillolide A (4)
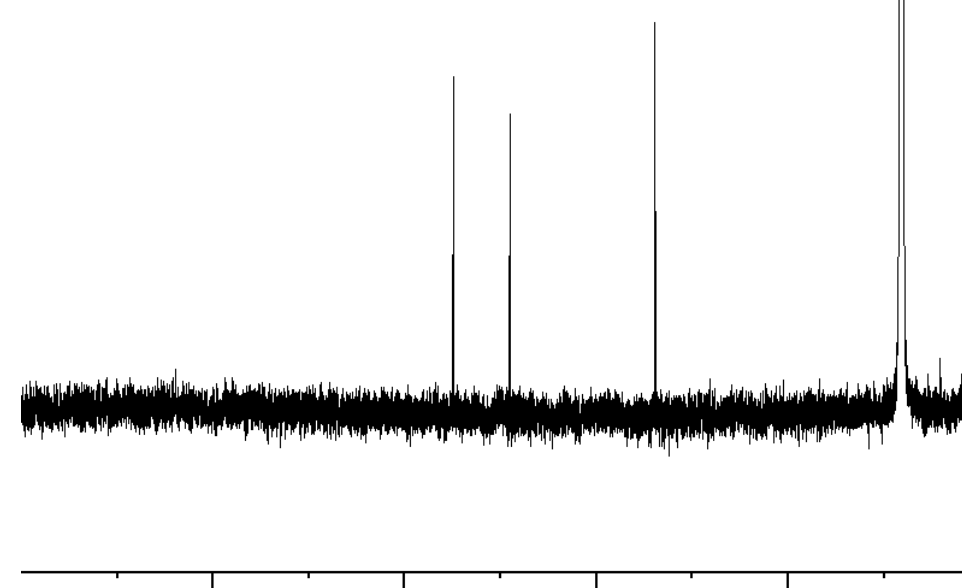